\title{
Electron bunchers for industrial RF linear accelerators: theory and design guide
}

\author{
Sergey V. Kutsaev ${ }^{\mathrm{a}}$ (D) \\ RadiaBeam Technologies LLC, 1717 Stewart St, Santa Monica, CA 90404, USA
}

Received: 28 September 2020 / Accepted: 13 March 2021

(C) The Author(s) 2021

\begin{abstract}
The acceleration of electrons in resonant linear accelerators (linacs) typically consists of three main stages: (1) emission of the electrons from the cathode and their preacceleration with a DC field to the energy of tens of $\mathrm{keV}$; (2) grouping the DC electron beam into bunches and their synchronization with the correct phase of high-frequency electromagnetic fields, and (3) accelerating the bunches of relativistic electrons to the required energies. Although many books describe the theoretical and practical aspects of electron linac design, most of them concentrate on beam physics in either the gun stage or in the relativistic regime, while leaving the description of the bunching process rather general. The physics of non-relativistic motion is described in the literature on ion accelerators, but in practice, it cannot be scaled to electron machines due to the significantly different particle mass and acceleration rate, beam velocity change, and frequencies. In this tutorial review paper, we will fill this gap with a detailed description of the bunching process and provide practical advice on the design of bunching sections in industrial-grade electron linacs.
\end{abstract}

\section{Introduction}

Particle accelerators are essential tools in nuclear and high-energy physics, medicine, material treatment, security, and industry. The progress in accelerator technologies was possible thanks to advancements in beam physics, radio frequency (RF) sources, material sciences, and fabrication techniques [1]. At present, accelerators provide beams of a wide range of charged particles, from electrons to radioactive isotopes, with energies from tens of $\mathrm{keV}$ to multiple $\mathrm{TeV}$, and currents from nA to $\mathrm{kA}$.

While "big science" accelerators such as the Large Hadron Collider [2] are the first ones to come to mind for a general reader, only $\sim 1 \%$ of all accelerators are large-scale machines for research [3]. In fact, 59\% of all accelerators are used for industrial and medical applications. A majority of them are electron linear accelerators with moderate energies of several to several tens of $\mathrm{MeV}$ (see Fig. 1). Modern accelerators must satisfy the requirements for reliability, economic efficiency, and suitability for the application (such as compactness, mobility, variability, etc.) [4], and these requirements evolve with the development of their applications. Therefore, it is essential to understand the physics of linear accelerators and the practical design aspects of their components.

\footnotetext{
a e-mail: kutsaev@radiabeam.com (corresponding author)
} 


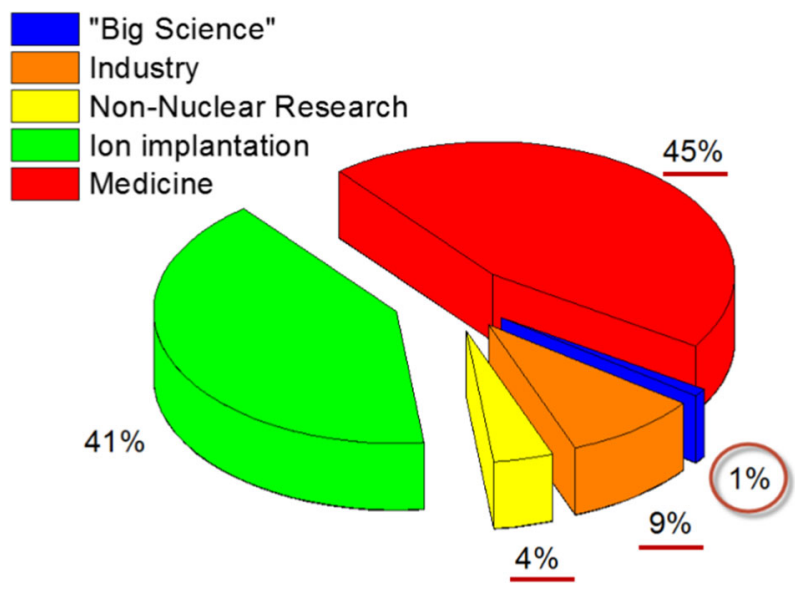

Fig. 1 Approximate proportion of accelerators used for various applications. Adapted from [3]

The acceleration of a particle with charge $q$ occurs due to the Coulomb force and interaction with the electric component $E$ of a DC or AC field as $\vec{F}=q \vec{E}$. Depending on the particle trajectory during acceleration, all accelerators can be divided into two main classes: circular and linear accelerators (linacs). In the latter case, the particle passes each accelerating gap only once on the way from the source to the target. Linear accelerators are also divided into subclasses, depending on the electric field source. One class is DC accelerators, where the particle energy grows due to the high voltage: electrostatic, cascade and transformer accelerators. [5] The second class is induction linacs, where the vertical electric force is caused by variation of the magnetic field in time [6]. The third group is resonant RF accelerators, where the particle gains energy by interacting with a high-frequency electromagnetic field [7]. There are also novel classes of accelerators where particles are accelerated by laser- or plasma-induced fields [8].

This tutorial paper's scope is limited to RF linear accelerators for industrial applications and does not cover the design aspects of scientific-grade linacs used as injectors in light sources. These accelerators have different requirements for the beam, focused on minimizing its size, and thus use different design philosophies. Also, unlike industrial machines, lightsource linac design is well covered in the literature [9].

The easiest way to describe the RF linac principle is by considering a traveling (TW) electromagnetic (EM) wave in a cylindrical waveguide. As known from the RF theory [10], such a wave can have a longitudinal component of the electric field, which is collinear with the beam trajectory, and therefore can accelerate the particles in phases where $E_{\mathrm{Z}} \cdot \cos \varphi>0$ (assuming $q>0$ ). In order to be accelerated consistently, the phase velocity of the EM field must synchronously increase with the particle velocity, so that it always remains in the accelerating phase, as shown in Fig. 2.

However, the phase velocity $\left(\beta_{\mathrm{ph}}{ }^{1}\right)$ of the EM wave in an unloaded (smooth-walled) waveguide is always higher than the speed of light, so it must be loaded with capacitive irises, becoming a so-called disk-loaded structure (DLS) or a corrugated waveguide, shown in Fig. 3. By varying the distance between these irises (structure period $L$ ), it is possible to achieve the required phase velocity profile. According to the Floquet theorem [11], the

${ }^{1}$ Here and throughout the text we will use the relative velocity $\beta$, which is defined as $\beta=\mathrm{v} / \mathrm{c}$, where $\mathrm{c}$ is the speed of light. 
Fig. 2 Principle of resonant (RF) acceleration. From top to bottom: the orientation of the electric field (red arrows) in an accelerating waveguide operating in the $\pi / 2$ mode, and bunch location (blue oval) at various times. T stands for RF period $1 / f$. The beam travels synchronously with the accelerating phase
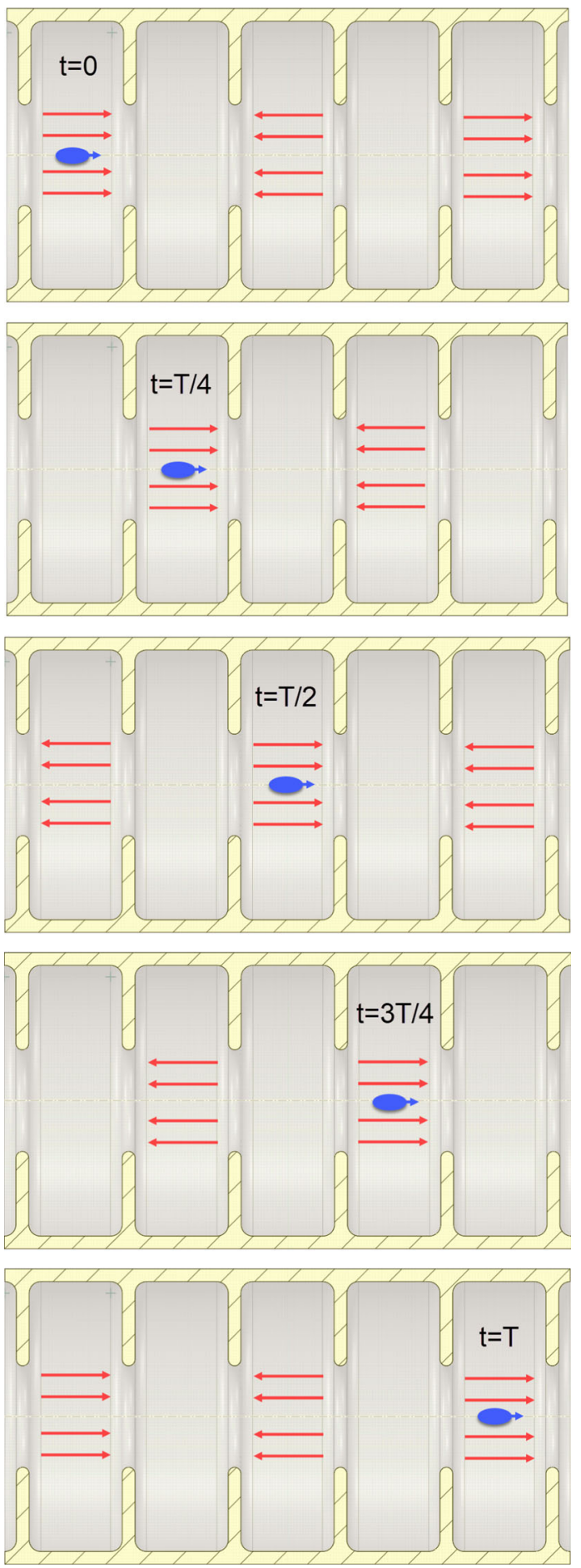
Fig. 3 Electric field (arrows) distribution at a given phase in a disk-loaded waveguide, operating at the $2 \pi / 3$ mode, as simulated in CST Microwave Studio (https:// www.3ds.com/products-services/ simulia/products/cst-studiosuite/). The phase changes by $2 \pi$ over a three-cell period. Here $L$ is the period of the structure, $a$ is the radius of the beam aperture, and $b$ is the radius of the DLS cell

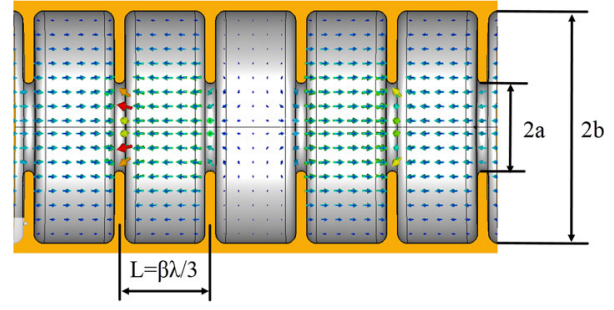

addition of irises modifies the waveguide's dispersion properties, allowing the propagation of waves with the same spatial distribution but different phase advance per structure period $\theta$. This phase advance, called the "acceleration mode", depends on the frequency and can vary from 0 to $\pi$. In practice, the preferred mode for a traveling wave guide is $2 \pi / 3$ due to the highest efficiency of energy transfer [12]. Sometimes $\pi / 2$ is preferred due to the simplicity of design and analytical calculations. In order to achieve a particular phase velocity, the period of DLS must satisfy the condition $L=\frac{\beta_{\mathrm{ph}} \lambda \theta}{2 \pi}$, where $\lambda$ is the wavelength in free space, which corresponds to the RF frequency $(f)$ as $\lambda=c / f$.

In periodic structures, the function of the electric field distribution $E(z)$ is also periodic with a period equal to the wavelength inside the structure and therefore can be expanded in Fourier series [14]:

$$
E_{z}(z)=\sum_{m=-\infty}^{\infty} E_{m} e^{\frac{-i \cdot m \cdot 2 \pi \cdot z}{L}} e^{i \cdot \omega \cdot t}=\sum_{m=-\infty}^{\infty} E_{m} e^{i \cdot\left(\omega \cdot t-k_{z, m} \cdot z\right)}
$$

According to this expression, the electric field in the periodic accelerating structure can be represented as the superposition of an infinite number of harmonic waves that propagate in different directions, with different phase velocities and amplitudes but the same oscillation frequency. These waves are called "spatial harmonics" and should not be confused with "time harmonics," which correspond to oscillations with different frequencies. Spatial harmonics have different values of phase velocities, defined as [14]:

$$
\beta_{\mathrm{ph}, m}=\frac{v_{\mathrm{ph}}}{c}=\frac{\omega}{k_{z, m}}=\frac{\omega}{k_{z, 0}+2 \pi m}
$$

Here $k_{\mathrm{z}}$ is the wave number. The harmonic with $m=0$ is called the "fundamental harmonic" and has the highest amplitude [15]. Because of this fact, the beam is usually synchronized and interacts with the fundamental harmonic; however, in some cases, operation on higher spatial harmonics can be beneficial due to lower phase velocities [16-18]. All further discussions are valid for any harmonic chosen for acceleration, which we will call the "accelerating harmonic."

Although the frequency of a DLS structure is a complex function of all dimensions, it is fair to say that it is mostly defined by the radius of the cylindrical waveguide (dimension $b$ in Fig. 3): smaller radii correspond to higher frequencies [10]. The beam aperture (a) defines the group velocity $\left(\beta_{\mathrm{gr}}\right)$ of the electromagnetic wave (collective velocity of all its spatial harmonics or energy propagation), which is related to the accelerating harmonic amplitude: smaller apertures correspond to higher accelerating field amplitudes [19]. The group velocity can be numerically found as [14]:

$$
\beta_{\mathrm{gr}}=\frac{d\left(\frac{1}{\lambda}\right)}{d\left(\frac{1}{\beta \lambda}\right)}=\frac{2 \pi L}{c} \frac{\mathrm{d} f}{\mathrm{~d} \theta}
$$


The efficiency of energy transfer from the EM wave to the beam in a DLS can be defined by the value of its shunt impedance per unit length $\left(r_{\mathrm{sh}}\right)$

$$
r_{\mathrm{sh}}=\frac{\left|\int_{0}^{L} E_{z}(z) \cdot e^{i k_{z} \cdot z} \mathrm{~d} z\right|^{2}}{P \cdot L}
$$

Here $L$ is the length of the accelerating structure, $P$ are power losses in a DLS waveguide walls, and $k_{z}=\frac{2 \pi}{\beta_{p h} \lambda}$ is the wave number. Another important parameter is the quality factor of the cavity that characterizes the ratio of the stored energy (W) to RF power losses:

$$
Q_{0}=\frac{\omega W}{P}
$$

The quality factor greatly depends on the cavity material and on the wall surface roughness, while the $r_{\mathrm{sh}} / Q$ ratio depends only on cavity geometry and thus characterizes the geometrical efficiency of the structure. The attenuation coefficient $\alpha$ can be calculated using the following formula and defines how the power of the EM wave attenuates along the structure $\left(P=P_{0} e^{-2 \alpha z}\right)$ :

$$
\alpha=\frac{\omega}{2 v_{\mathrm{gr}} Q}
$$

Finally, one of the most important parameters that relates the electric field amplitude of accelerating harmonic (E) with its RF parameters (usually found numerically) is the so-called normalized electric field amplitude:

$$
\Lambda=\frac{E \lambda}{\sqrt{P}}=\sqrt{\frac{2 \pi \lambda r_{s h}}{Q \beta_{g r}}}
$$

These parameters will be used in the following sections where we will discuss the practical steps for buncher designs.

As mentioned above, this paper is focused on industrial-grade electron linacs and does not cover ion accelerators, where the requirements for the beam, the accelerator design philosophy and the tools are different [20]. The difference between an electron and hadron acceleration comes from their rest-energy $\left(W_{0}=0.511 \mathrm{MeV}\right.$ for electrons and $\sim 931.5 \mathrm{MeV}$ for a single hadron). Due to the light mass, the same energy gain for the electrons leads to a much larger velocity gain: $\beta=\frac{v}{c}=\frac{\sqrt{\gamma^{2}-1}}{\gamma}, \gamma=1+\frac{W}{W_{0}}$. Here $W$ is the particle's kinetic energy, and the electron's energy approaches nearly the speed of light already at $\sim 1 \mathrm{MeV}$. Table 1 and Fig. 4 show the beam velocities for different values of the beam energies and compares these values to those for protons.

The acceleration process in a DLS-based electron linac consists of the following steps, as shown in Fig. 5. Electrons are emitted from the cathode (usually, thermionic), pre-accelerated in a DC gun to energies of several tens of $\mathrm{keV}$ (sometimes to over a hundred $\mathrm{keV}$ in highcurrent accelerators) and are injected into the front-end of the DLS waveguide [21]. At this point, the electron beam is continuous, and its speed is well below the speed of light (usually, $0.2-0.5 \mathrm{c}$ ). The particles of this DC beam arrive at the EM field's different phases, and their energy distribution starts to change. The continuous beam transitions into small bunches in an adequately designed accelerator due to large phase oscillations. This part is usually referred to as the "buncher" and can be either a separate section or integrated into the accelerating section.

The linac may also utilize a resonator with a small RF voltage placed after the DC gun and separated from the buncher by a drift space in order to provide velocity modulation, 
Table 1 Comparison of the electron and proton energies, corresponding to different velocities

\begin{tabular}{lcr}
\hline Velocity $\beta$ & \multicolumn{2}{l}{ Energy, MeV } \\
\cline { 2 - 3 } & Electron & Proton \\
\hline 0.4 & 0.047 & 85 \\
0.5 & 0.079 & 146 \\
0.6 & 0.128 & 235 \\
0.7 & 0.205 & 376 \\
0.8 & 0.341 & 627 \\
0.9 & 0.661 & 1220 \\
0.99 & 3.12 & 5720 \\
0.999 & 10.95 & 20,100
\end{tabular}

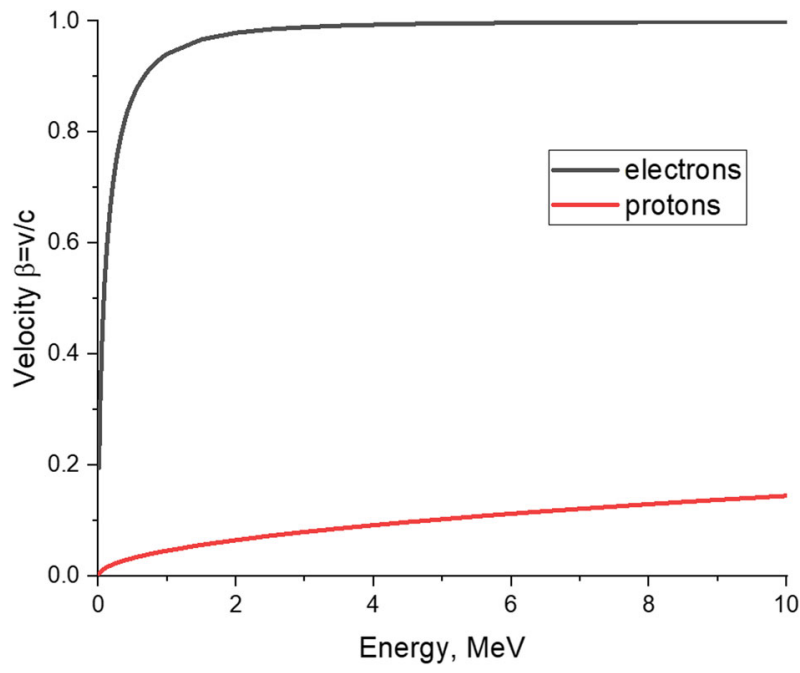

Fig. 4 Electron (black) and proton (red) velocities as a function of their kinetic energies

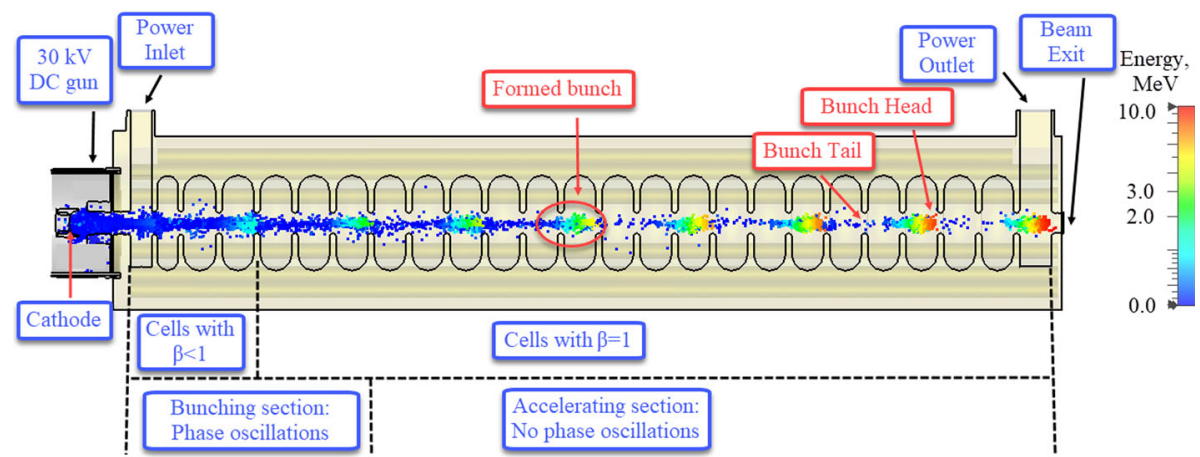

Fig. 5 Acceleration of particles in a DLS-based TW electron linac, as simulated in CST Particle Studio [13]. From left to right: the electrons are emitted from the cathode, pre-accelerated in the gun, bunched in a three-cell waveguide buncher, and accelerated in the $\beta=0.999$ constant impedance section 
which turns into density modulation of the beam after passing through the drift (so-called ballistic bunching). In this case, such a resonator is usually referred to as a "pre-buncher." To distinguish a ballistic buncher from a buncher that forms part of the accelerating structure, the latter is usually referred to as a "waveguide buncher." The bunching typically ends at energies of $\sim 1-3 \mathrm{MeV}$, and the beam continues its acceleration in the DLS section with $\beta_{\mathrm{ph}}$ $\sim 1$ until the particles reach the required energy. Since the electrons are ultra-relativistic at this stage, their velocity changes insignificantly, and the phase motion is essentially frozen. This part of the linac is usually called the "accelerating section."

It is also worth mentioning that although we mostly consider DLS-based linacs in this work, a similar concept is applicable for other types of slow-wave structures such as dielectricloaded structures. In the following text, we will use the term "accelerating structure" or "accelerating waveguide" to mean any RF waveguide structure with phase velocity $\beta_{\mathrm{ph}} \leq 1$ and with a field configuration suitable for transferring energy from the EM wave to particles (i.e., with the electric field collinear to the beam direction).

The length of the bunching section depends on the EM field strength. For high gradient accelerators, the buncher can consist of only a few cells or even a single $1 / 2$-cell. On the contrary, low-energy accelerators $(<1 \mathrm{MeV})$ with low available RF power can consist entirely of a bunching section. Also, according to the fundamental theorem of beam loading [22], the beam passing through the accelerating structure not only takes the energy of the EM wave but also excites one. This beam-excited wave adds to the RF wave and can change its amplitude and phase (the so-called beam loading effect). Beam loading can be substantial during the bunching and acceleration of high-current beams.

The bunching efficiency is characterized by a "capture coefficient" $k_{C}$, which can be defined by the number of particles accepted to the acceleration regime (within the RF bucket as will be shown in the following section), related to the number of particles injected from the gun within a single RF period. This parameter should not be confused with the "transmission coefficient" $k_{T}$ defined as the ratio of the beam current at the linac exit to the injected current. The second parameter also accounts for the particles that are not captured into the steady acceleration regime but slip along the EM wave while still reaching the end of the linac, as well as for the particle losses not related to the bunching mechanism (higher harmonics, deflection, external forces, etc.).

In the following sections, we will provide the mathematical model of particle motion in a corrugated waveguide and introduce the phase-space concept and its dependence on the accelerating waveguide parameters, which is a handy tool for the buncher design. The provided material is based on Russian and Soviet books [23-29] and publications that are neither available in electronic format nor in English.

Section 2 is devoted to a theoretical background of buncher operation and includes the fundamentals of longitudinal motion, a description of the phase space concept, phase and velocity acceptance, as well as the capture and bunching processes. It will also discuss what role accelerating waveguide and beam parameters play on the beam dynamics and bunching process, and what are the practical limitations on their choice. In the end of the section, we will discuss the differences between the beam dynamics approach in standing and traveling wave linacs and demonstrate the applicability of the presented model for both types.

In Sect. 3, we will move from the theory of electron bunchers to their practical realization. We will briefly overview different types of electron bunchers that are typically used in industrial linacs, describe their general pros and cons, followed by an introduction of analytical models and practical guidance for the design, including some design examples. Finally, we will discuss some practical aspects of beam acceleration, such as phase slippage and frequency mismatch effects. 
This paper does not attempt to review all buncher designs that were ever proposed by accelerator scientists, but rather to present different design philosophies, possible options and trade-offs, so that the reader can use any of the presented techniques in the design process. We believe that this tutorial would be interesting to graduate students studying particle accelerators and accelerator physicists who are engaged in industrial accelerator design, as well as the general audience interested in expanding their knowledge in accelerator physics.

\section{Theory of electron bunchers}

\subsection{Phase space and oscillations}

As discussed in the previous section, although high-frequency structures and low-beta beams can produce strong coupling of the longitudinal and transverse motions, we may ignore this coupling for the simplicity of the theory. In this case, the capture efficiency can be calculated by considering only the longitudinal (phase) motion of the beam [30]. There are a lot of books covering this problem, including but not limited to [31-40]. In this section, we will provide a brief overview of the fundamentals of longitudinal beam dynamics, introduce the principal parameters, such as normalized field, velocity and coordinates, describe the concept of phase space, which will be followed by theory of bunching, concepts of beam acceptance and the practical limitations of buncher design that will serve the basis for the later chapters.

The fundamental equation of particle motion, $\frac{d \vec{p}}{d t}=q \vec{E} \cos \varphi$, can be rewritten as the energy gain along the longitudinal coordinate $z$, since $\frac{\mathrm{d} p_{z}}{\mathrm{~d} t}=\frac{\mathrm{d} W}{\mathrm{~d} z}$ :

$$
\frac{\mathrm{d} W}{\mathrm{~d} z}=q E_{z} \cos \varphi
$$

Let us introduce the following normalized parameters: energy $\gamma=\frac{W}{W_{0}}$, the phase velocity of the wave $\beta_{\mathrm{ph}}=\frac{v_{\mathrm{ph}}}{c}$, the longitudinal coordinate $\zeta=\frac{z}{\lambda}$, the amplitude of the accelerating harmonic (usually the fundamental harmonic but there are examples of structures operating at higher harmonics [17]) of the EM wave $A=\frac{\mathrm{qE} \lambda}{W_{0}}$. Now, Eq. (8) can be written as:

$$
\frac{\mathrm{d} \gamma}{\mathrm{d} \zeta}=A \cos \varphi
$$

and the particle phase shift due to its velocity $(\beta)$ mismatch to the wave velocity as:

$$
\frac{\mathrm{d} \varphi}{\mathrm{d} \zeta}=2 \pi\left(\frac{1}{\beta_{\mathrm{ph}}}-\frac{1}{\beta}\right)
$$

Taking into account that $\beta=\frac{\sqrt{\gamma^{2}-1}}{\gamma}$, we can combine Eqs. (9) and (10) into one:

$$
2 \pi\left(\frac{1}{\beta_{\mathrm{ph}}}-\frac{\gamma}{\sqrt{\gamma^{2}-1}}\right) \mathrm{d} \gamma=A \cos \varphi \cdot \mathrm{d} \varphi
$$

Assuming that both the amplitude and phase velocity of the accelerating waveguide remain constant in some regions (for example, one cell), then we can integrate Eq. (11) and get:

$$
\frac{\gamma}{\beta_{\mathrm{ph}}}-\sqrt{\gamma^{2}-1}=\frac{A}{2 \pi} \sin \varphi+H
$$


Here $H$ is a constant of integration. By replacing $\gamma$ with $\beta$, we can finally get:

$$
\frac{1-\beta_{\mathrm{ph}} \beta}{\sqrt{1-\beta^{2}}}-\frac{A \beta_{\mathrm{ph}}}{2 \pi} \sin \varphi=H \beta_{\mathrm{ph}}=H_{1}=\mathrm{const}
$$

Equation (13) relates the change of electron's phase with the change of its velocity during its motion in the RF field. In the case of $\beta_{\mathrm{ph}}<1$, there are several electron motion patterns, depending on its initial phase and velocity. When the particle is much faster than the wave, and the amplitude is small, the particle will always slide through the wave with a phase change more than $2 \pi$. A similar pattern would be observed if the particle is too slow, relative to the wave, so it always falls behind it. The most interesting case is when the electron moves along with the EM wave, and its phase oscillates around the phase of the electromagnetic wave.

The described cases can be visually demonstrated in the plot with the phase as the horizontal axis and the energy (or momentum) as the vertical axis-a so-called phase space plot. Different values of $H_{1}$ in formulae (13) will correspond to different trajectories representing particles with different initial phases and velocities. An example of such trajectories is shown in Fig. 6. At the bottom of the figure, we show the accelerating field profile that corresponds to these phases. During the electron motion through the waveguide, the particles move clockwise along these trajectories. The direction of motion along the trajectories is defined by the condition that $\frac{\mathrm{d} \varphi}{\mathrm{d} z}>0$ for $p>0$.

Point (a) in the phase space corresponds to an electron with a velocity equal to the wave's phase velocity and a phase equal to $\varphi_{\mathrm{s}}=\pi / 2(E=0)$. This electron will move synchronously with the wave since its energy (speed) does not change. Electron (b) is initially slightly slower than the wave. Therefore, it will slip behind the $\pi / 2$ phase into the accelerating region $(E$ $>0$ ) and acquire energy. At some point, its velocity will be higher than the wave's velocity, and the electrons will surpass it, finding itself in the decelerating wave and losing its energy. This electron will oscillate around the equilibrium phase $\pi / 2$.

If the electron velocity is too low (c) or too high (d), the time they spend in accelerating or decelerating phases is not enough to change the direction of phase motion, and it will always slip along the RF phase. These particles will not be captured into the accelerating regime and will eventually be lost. The border between stable and unstable trajectories is called the separatrix, and the area inside the separatrix is called the RF bucket. The particles inside the RF bucket will form a bunch. From the phase trajectories in Fig. 6, it is evident that in accelerating waveguides with $\beta_{\text {ph }}<1$, the electrons' energy cannot grow beyond a specific value, even in an infinitely long structure. It is necessary to modify the RF bucket by increasing its phase velocity and amplitude along the accelerating waveguide so that its equilibrium energy grows with the beam's energy.

Figure 7 shows the evolution of RF bucket shape with increasing phase velocity and constant amplitude. By integrating Eq. (13) and taking into account that the separatrix crosses the point $\left(-\pi / 2, \beta_{\mathrm{ph}}\right)$, it is possible to find the maximum energy height of the separatrix, called "energy acceptance" $[10,25]$ :

$$
\Delta \gamma_{\max }=2 \sqrt{\frac{2 A\left(\beta_{\mathrm{ph}} \gamma_{\mathrm{ph}}\right)^{3}}{\pi}}=\sqrt{\frac{8 A\left(\frac{\beta_{\mathrm{ph}}^{2}}{1-\beta_{\mathrm{ph}}^{2}}\right)^{3 / 2}}{\pi}}
$$

The area of the RF bucket in the phase space is related to the energy acceptance as $A_{\mathrm{RF}}=4 \Delta \gamma_{\max }[22]$. Both values progressively increase with the phase velocity of the EM wave and the amplitude of the accelerating harmonic. The phase acceptance depends on the injection energy and will be discussed in the following chapter. 

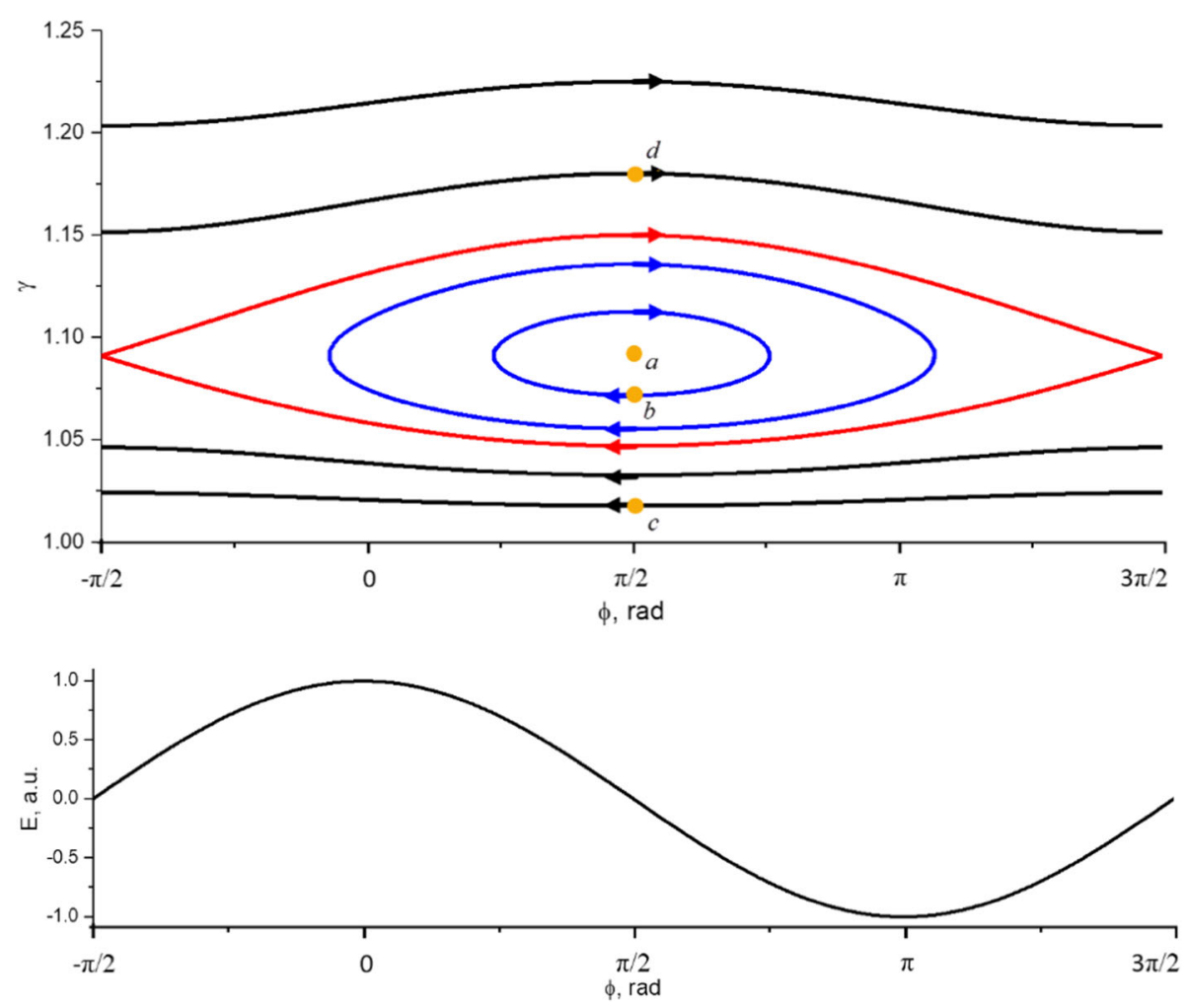

Fig. 6 Top: phase trajectories for particles with different velocities (yellow dots marked by letters) moving in an EM wave with $A=0.05$ and $\beta_{\mathrm{ph}}=0.4$. The red curve is the boundary of stable oscillation regions-the separatrix. Blue trajectories are stable, electrons on black trajectories are lost. Bottom: Amplitude of the electric field of the EM wave as a function of the RF phase

Now, it is interesting to calculate the phase trajectories of electrons in extreme cases when the phase velocity of the EM wave is equal to the speed of light. Such sections can be used to accelerate ultra-relativistic electrons since their speed changes insignificantly with energy gain (see Table 1), and therefore, they can interact with the EM wave for a long time. Assuming that the RF field amplitude does not decay during its propagation in the accelerating waveguide, Eq. (12) evolves into:

$$
\gamma-\sqrt{\gamma^{2}-1}=\frac{A}{2 \pi} \sin \varphi+H_{1}
$$

This equation allows plotting phase trajectories, as shown in Fig. 8. We see that there is no equilibrium phase for $\beta_{\mathrm{ph}}=1$ structures as well as no RF bucket: the closed trajectories that were within a separatrix are now deformed into lines that have vertical asymptotes. In this case, the trajectory (2) plays the role of the separatrix. Trajectories (3) correspond to particles accepted for acceleration. The particles moving along trajectories (1) always stay behind the beam and either form a bunch tail or are lost.

The value of the asymptotic phase can be found considering that $\frac{A}{2 \pi} \sin \varphi+H_{1}=0$ :

$$
\varphi_{\mathrm{as}}=a \sin \left(\sin \varphi_{0}-\frac{2 \pi}{A} \sqrt{\frac{1-\beta_{0}}{1+\beta_{0}}}\right)
$$




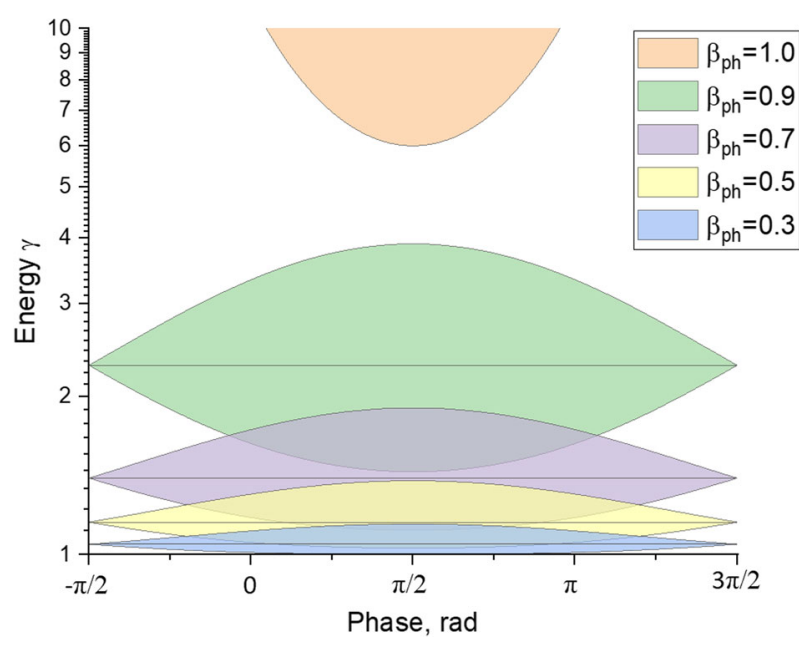

Fig. 7 Evolution of the RF bucket as a function of phase velocity from 0.3 to 1.0 for $A=0.25$. It is clearly seen that the energy acceptance and maximum energy increases

It is now possible to tell what injection phase $\varphi_{0}$ is best in terms of acceleration efficiency in a $\beta_{\text {ph }}=1$ accelerating waveguide. Unlike the $\beta_{\text {ph }}<1$ case, there are no closed trajectories, meaning that the particles do not oscillate around the synchronous phase. In that view, even the "unstable" trajectories (1) outside the asymptotic trajectory (2) can accelerate particles to some limited energy. The separatrix trajectory does not have this limit, but during acceleration, the particle will slide toward the asymptotic phase of $\varphi=-\pi / 2$, which corresponds to a lowfield region, thus low acceleration efficiency. For trajectories (3), the asymptotic phase is zero (wave crest), and the acceleration is most efficient. Unlike an ion beam, where the synchronous phase must be different from zero, the electron beam is accelerated most efficiently when the bunches are located on the crest.

\subsection{Phase acceptance}

The intensity of the accelerated particles depends on the intensity of the particle source, the number of particles captured into the accelerating regime, and the number of particles transmitted through the structure (i.e., considering transverse losses). In this paper, we will only consider the longitudinal motion of the beam, i.e., no transverse losses. Let us assume that the beam is injected from a DC gun; then the injected particles in phase space will represent a continuous line with a constant $\gamma$ and phases ranging from $-\pi / 2$ to $3 \pi / 2$ (red line in Fig. 9). This plot shows that only the particles in the phase range $\left[\varphi_{1}, \varphi_{2}\right]$ lie within the RF bucket. These particles will be travelling along with the EM wave. Electrons located outside this region will slide relative to the wave and eventually be lost. Therefore, we can define the capture coefficient as $k_{C}=\frac{\varphi_{2}-\varphi_{1}}{2 \pi}$.

Now we can find the values of $\varphi_{1}$ and $\varphi_{2}$ from Eq. (13). To do this, we will define the value of $\mathrm{H}_{1}$ for the separatrix taking into account that the phase velocity $\beta_{\mathrm{ph}}$ and the amplitude $\mathrm{A}$ are defined. Then, for $\varphi=-\pi / 2$ (or $3 \pi / 2$ ) and $\beta=\beta_{\mathrm{ph}}$ :

$$
H_{\mathrm{sep}}=\sqrt{1-\beta_{\mathrm{ph}}^{2}}+\frac{A \beta_{\mathrm{ph}}}{2 \pi}
$$



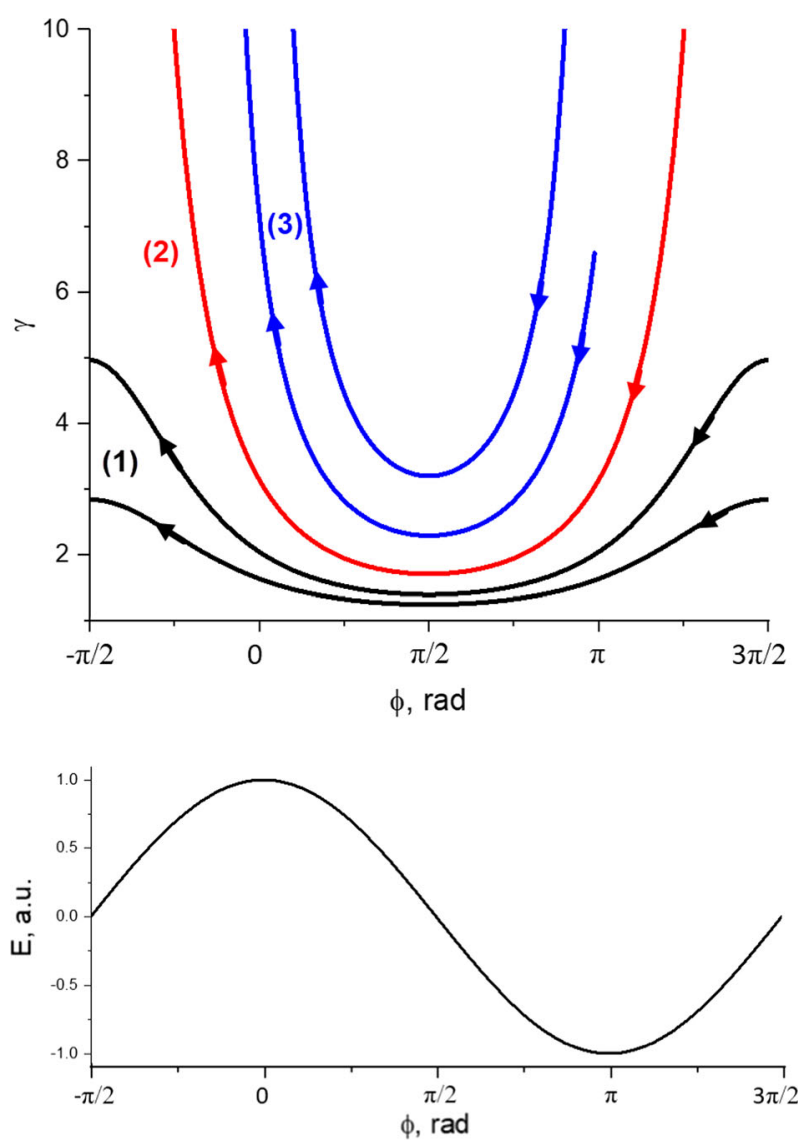

Fig. 8 Top: phase trajectories for particles with different velocities, moving in EM wave with $A=1$ and $\beta_{\mathrm{ph}}=1$. The red curve is a quasi-separatrix. Blue trajectories are in the stable acceleration regime; electrons on black trajectories form a beam tail or are lost. Bottom: amplitude of the electric field of the EM wave as a function of the RF phase

This value, along with the known injection beam energy, can now be applied to Eq. (13) so that

$$
\sqrt{1-\beta_{\mathrm{ph}}^{2}}+\frac{A \beta_{\mathrm{ph}}}{2 \pi}=\frac{1-\beta_{\mathrm{ph}} \beta_{\mathrm{inj}}}{\sqrt{1-\beta_{\mathrm{inj}}^{2}}}-\frac{A \beta_{\mathrm{ph}}}{2 \pi} \sin \varphi_{1}
$$

and finally:

$$
\varphi_{1}=\operatorname{asin}\left[\frac{2 \pi}{A \beta_{p h}}\left(\frac{1-\beta_{p h} \beta_{i n j}}{\sqrt{1-\beta_{i n j}^{2}}}-\sqrt{1-\beta_{p h}^{2}}\right)-1\right]
$$


Fig. 9 Phase space of a $35 \mathrm{keV}$ DC beam (red line), injected into a ion with $\beta_{\mathrm{ph}}=0.3$. The particles with phases outside the range of $[\varphi 1, \varphi 2]$ will not be captured. The yellow line corresponds to the case of $\beta_{i n j}=$ $\beta_{\text {ph }}$ and maximum capture. A beam injected below the threshold energy (magenta line) will not be accepted to acceleration

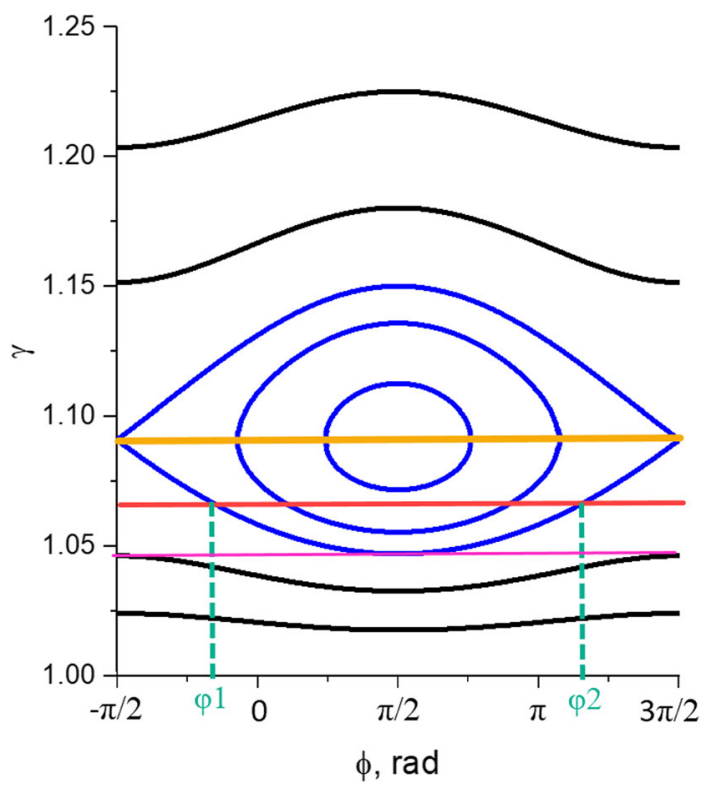

Since $\varphi_{1}$ and $\varphi_{2}$ are symmetric around $\pi / 2\left(\varphi_{2}=\pi-\varphi_{1}\right)$, the capture coefficient $k_{C}=\frac{\left(\pi-\varphi_{1)}-\varphi_{1}\right.}{2 \pi}$ can be expressed as:

$$
k_{C}=\frac{1}{2}-\frac{1}{\pi} a \sin \left[\frac{2 \pi}{A \beta_{\mathrm{ph}}}\left(\frac{1-\beta_{\mathrm{ph}} \beta_{\mathrm{inj}}}{\sqrt{1-\beta_{\mathrm{in} j}^{2}}}-\sqrt{1-\beta_{\mathrm{ph}}^{2}}\right)-1\right]
$$

The maximal capture $\left(k_{\mathrm{C}}=1\right)$ is achieved when $\beta_{\text {inj }}=\beta_{\text {ph }}$ (yellow line in Fig. 9). We would like to note that particles are rarely injected with the $\beta_{\text {inj }}>\beta_{\text {ph }}$ since the beam, in this case, will fall into the deceleration phase very quickly, thus making such injection unreasonable for general purposes. The purple line defines the threshold injected energy of the beam, below which no part of the beam is accepted. Therefore, for a given injection energy of the beam and phase velocity of the waveguide cell, it is possible to find the minimal value of RF field amplitude where beam capture is possible. To do this, we solve Eq. (20) for $k_{\mathrm{C}}$ $=0$ (purple line in Fig. 9, touching the separatrix at the single point $\varphi=\pi / 2$ ):

$$
A_{\min }=\frac{\pi}{\beta_{\mathrm{ph}}}\left(\frac{1-\beta_{\mathrm{ph}} \beta_{\mathrm{inj}}}{\sqrt{1-\beta_{\mathrm{inj}}^{2}}}-\sqrt{1-\beta_{\mathrm{ph}}^{2}}\right)
$$

It is worth mentioning that when the phase velocity is matched to the beam velocity $\beta_{\text {ph }}$ $=\beta_{\text {inj }}$, capture is possible for any field value. Also, some accelerators do not have bunching ions at all, and the electrons are injected directly into the ion with $\beta_{\mathrm{ph}}=1$. In this case, the field amplitude must be higher than:

$$
A_{\min }=\pi \sqrt{\frac{1-\beta_{\text {inj }}}{1+\beta_{\text {inj }}}}
$$



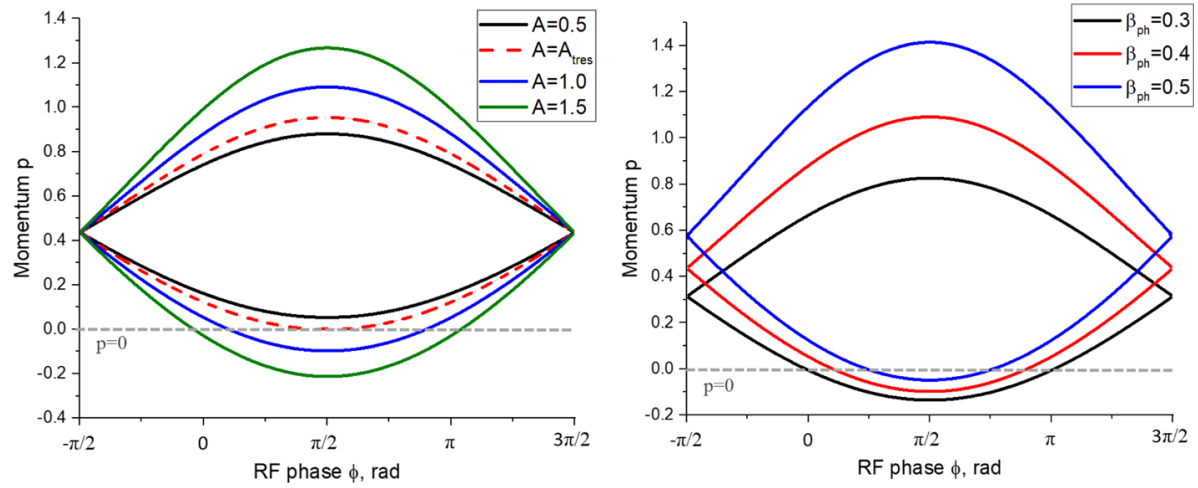

Fig. 10 Separatrix shapes for different values of field amplitude and phase velocities. Left: $\beta_{\mathrm{ph}}=0.4$, right: $A=1$

\subsection{RF amplitude threshold}

Equation (20) is valid only for specific conditions: if A is very high, some electrons will be decelerated and reverse their direction of motion. In this case, it is convenient to use a momentum-based phase space instead of energy-based, since momentum can be negative, unlike $\gamma$. Therefore, taking into account the expression for normalized momentum $p=\beta \gamma$, Eq. (13) can be written in the form of:

$$
H_{1}=\frac{1}{p_{0}+\sqrt{p_{0}^{2}+1}}-\frac{A}{2 \pi} \sin \varphi_{0}
$$

If we plot the phase trajectories in this phase space, we can observe that the separatrix stretches with increasing field amplitude and can reach $p=0$ at $\varphi=\pi / 2$ for some value of $A_{\text {th }}$-see Fig. 10. Further increasing the RF field amplitude will lead to the situation where the RF bucket will partially occupy the area with $p<0$, as shown in Fig. 11. The future of these particles will depend on the particular design of the accelerator, but most likely they will eventually be rejected back to the gun [25]. Although in principle in the case of a negative momentum some small fraction of particles can be captured into the RF bucket, we assume them to be lost for the purposes of a conservative design approach. In this case, only the particles that move along the trajectories that do not cross the $p=0$ line will be captured into the accelerating regime.

The amplitude that defines the threshold of this effect $\left(A_{\mathrm{th}}\right)$ can be calculated by assuming $\beta_{\text {inj }}=0$ in Eq. (21), which results in the following value:

$$
A_{\mathrm{th}}=\frac{\pi}{\beta_{\mathrm{ph}}}\left(1-\sqrt{1-\beta_{\mathrm{ph}}^{2}}\right)
$$

The border trajectory within which particles are captured touches the point $p=0$ (or $\beta=0$ ) at $\varphi=\pi / 2$. For this point, we can use Eq. (13) to determine the constant $H$ :

$$
H_{1}=1-\frac{A \beta_{\mathrm{ph}}}{2 \pi}
$$


Fig. 11 Phase trajectories within an RF bucket (red line) in the momentum-phase coordinates for a ion with $\beta_{\mathrm{ph}}=0.4$ and $\mathrm{A}=3.0$. Electrons moving along dashed trajectories will acquire negative momentum and will be rejected from the acceleration. Only solid green trajectories are suitable for capture

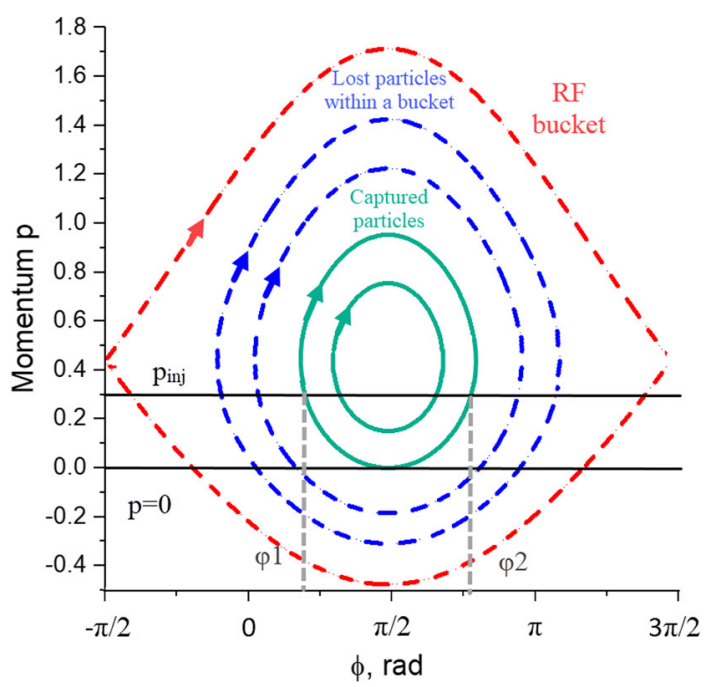

Now it is possible to solve this equation for the border phases $\varphi_{1}$ and $\varphi_{2}$, similar to what we did for Eq. (20):

$$
\begin{gathered}
\frac{1-\beta_{\mathrm{ph}} \beta_{\mathrm{inj}}}{\sqrt{1-\beta_{\mathrm{inj}}^{2}}}-\frac{A \beta_{\mathrm{ph}}}{2 \pi} \sin \varphi_{2}=1-\frac{A \beta_{\mathrm{ph}}}{2 \pi} \\
\varphi_{2}=\pi-a \sin \left[\frac{2 \pi}{A \beta_{\mathrm{ph}}}\left(\frac{1-\beta_{\mathrm{ph}} \beta_{\mathrm{inj}}}{\sqrt{1-\beta_{\mathrm{inj}}^{2}}}+\frac{A \beta_{\mathrm{ph}}}{2 \pi}-1\right)\right]
\end{gathered}
$$

Finally, we can calculate the capture coefficient

$$
\begin{aligned}
k_{C}= & \frac{1}{2 \pi}\left\{a \cos \left[\frac{2 \pi}{A \beta_{\mathrm{ph}}}\left(\frac{1-\beta_{\mathrm{ph}} \beta_{\mathrm{inj}}}{\sqrt{1-\beta_{\mathrm{inj}}^{2}}}+\frac{A \beta_{\mathrm{ph}}}{2 \pi}-1\right)\right]\right. \\
& \left.+a \cos \left[\frac{2 \pi}{A \beta_{\mathrm{ph}}}\left(\frac{1-\beta_{\mathrm{ph}} \beta_{\mathrm{inj}}}{\sqrt{1-\beta_{\mathrm{inj}}^{2}}}-\sqrt{1-\beta_{\mathrm{ph}}^{2}}\right)-1\right]\right\}
\end{aligned}
$$

According to this formulae, the capture coefficient is maximized for $A=A_{\text {th }}$ and converges to $k_{\mathrm{C}}=0.5$ at $A \rightarrow \infty$, when only particles within phase [ $\left.-\pi / 2, \pi / 2\right]$ are captured. Figure 12 plots the values of threshold amplitudes for cells with different phase velocities. The dependences of the capture coefficient on the field strength for different injection energies are shown in Fig. 13. Injection of low-energy beams into a structure with high phase velocity can lead to significant particle losses and the transmission is limited to below $50 \%$ if the amplitude is chosen incorrectly. Moreover, when the RF power is also limited $\left(A<A_{\mathrm{th}}\right)$, it is particularly important to choose the proper phase velocity due to the high sensitivity of capture to the RF field and the risks of complete beam rejection. 


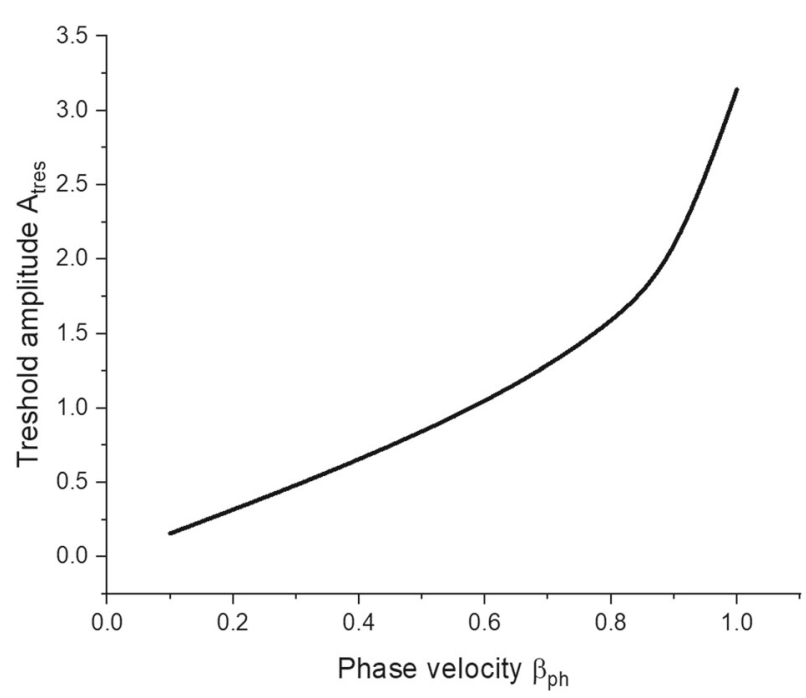

Fig. 12 Threshold amplitudes for cavities with different phase velocities

\subsection{Practical limitations for beam injection}

In this paragraph, we discuss the choice of the injection and phase velocity parameters and, particularly, their practical limitations. In order to achieve $100 \%$ capture of the electrons, the following conditions must be met:

$$
\begin{gathered}
\beta_{\mathrm{inj}}=\beta_{\mathrm{ph}} \\
\left.\frac{d \beta_{\mathrm{ph}}}{d z}\right|_{z=0}=0 \\
A \leq \frac{\pi}{\beta_{\mathrm{ph}}}\left(1-\sqrt{1-\beta_{\mathrm{ph}}^{2}}\right)
\end{gathered}
$$

In practice, some of these conditions can be hard or impossible to achieve. First, the phase velocity of the DLS structure has a lower limit of $\beta_{\mathrm{ph}}>0.3$ due to manufacturing challenges and poor electrodynamic performance. According to Fig. 3, the phase velocity of the DLS is proportional to its length, so the irises must be put closer to each other in order to reduce $\beta_{\mathrm{ph}}$. The physical limit of phase velocity occurs when these irises become so close to each other to cause electric breakdown (this minimum distance depends on the accelerating gradient). However, the practical limit is driven by the dramatic deterioration of its efficiency (the ratio of energy transferred to the beam to the power dissipated in the walls). Figure 14 demonstrates how the accelerating field amplitude A, normalized to the RF input power and wave attenuation per unit length, depends on the phase velocity [41]. Long ions with low velocity can significantly reduce the available RF power, which will reduce the accelerator efficiency or even require the utilization of expensive high-power RF sources.

Other considerations for minimum phase velocity include fabrication feasibility: low- $\beta$ cells have larger radii, and much larger radius-to-length ratio [58], which can be challenging to machine (for example, in a split structure DLS [42, 43]), or might not have space to place frequency tuners [17]. The practical limit of $\beta_{\mathrm{ph}}$ is $\sim 0.3$ for S-band structures but is usually set at 0.5-0.6 for higher-frequency or compact industrial accelerators [21, 44]. 
(a)

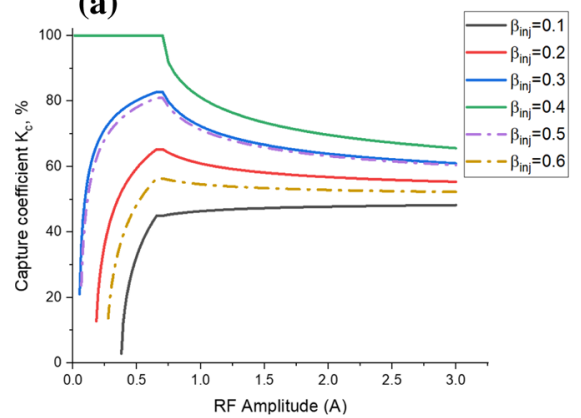

(c)

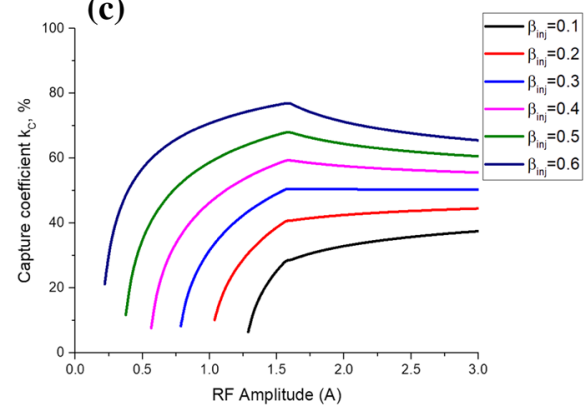

(b)

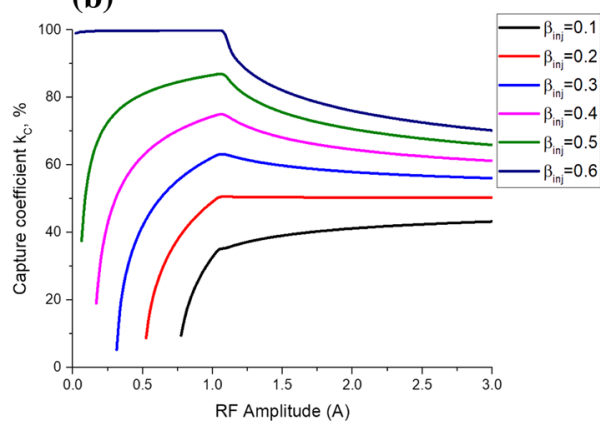

(d)

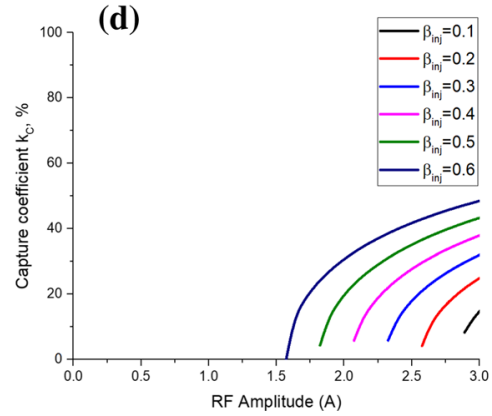

Fig. 13 Capture coefficient $\mathrm{kC}$ as a function of field amplitude $\mathrm{A}$ in an accelerating waveguide for different beam injection energies and phase velocities of the EM wave: $\mathbf{a} \beta_{\mathrm{ph}}=0.4 ; \mathbf{b} \beta_{\mathrm{ph}}=0.6 ; \mathbf{c} \beta_{\mathrm{ph}}=0.8 ; \mathbf{d} \beta_{\mathrm{ph}}$ $=1.0$. The discontinuity in the plot derivatives corresponds to the threshold field amplitude $A_{\text {tres }}$ values, presented in Fig. 12
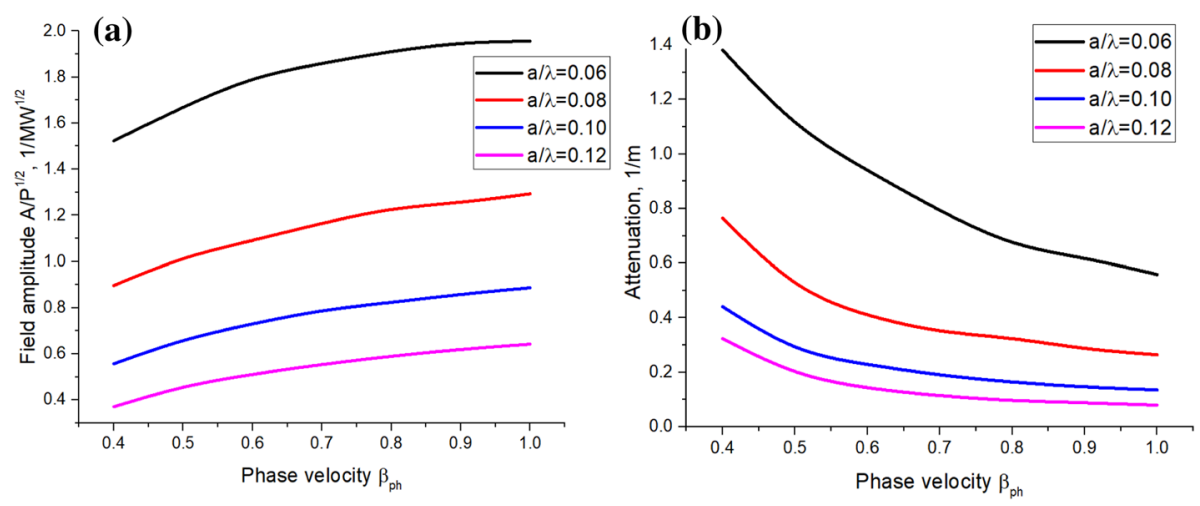

Fig. 14 RF parameters of DLS waveguides for different phase velocities and beam apertures (refer to Fig. 3): left-field amplitude A, normalized to the square root of RF power; right-attenuation decrement for an S-band DLS

The choice of high phase velocities leads to the requirement of beam injection with the same velocity, according to (29). At the same time, higher injection voltages are more preferable in terms of beam dynamics in electron guns: higher-energy beams are less prone to space charge effects $[38,45,46]$, and the maximum current that can be extracted from a cathode is proportional to gun voltage according to Child's law: $I \sim P \cdot V_{3 / 2}$ [47]. Here $P$ is 
the gun's perveance, defined by the geometry of the gun. However, the price, dimensions and complexity of DC electron guns and power supplies grow significantly with the voltage $[44,48]$, (mostly, due to isolation and pulsed power system complexity), and in practice are preferred to be kept around $10-20 \mathrm{kV}$ for compact medical accelerators, $30-50 \mathrm{kV}$ for industrial accelerators and to $80-160 \mathrm{kV}$ for large high-current facilities [15, 21, 44, 49-51]. Therefore, the injection usually occurs at lower velocities than $\beta_{\mathrm{ph}}$, which fundamentally limits the capture of the beam.

In practice, electron linacs are usually not designed for very high capture ratios since it is usually more efficient to compensate electron losses by increasing injection currents, rather than by reducing the accelerator efficiency by introducing complex bunching systems. For example, with enough RF power, it is possible to achieve $>60 \%$ capture in short bunching ions even with a rapid phase velocity increase [52-54]. For some industrial machines, even $30-40 \%$ transmission can be acceptable, simplifying the buncher design to 1-3 cells [44]. The following chapters will describe different buncher types and bunching techniques in more detail and provide some practical guidance for the buncher design.

\subsection{Traveling and standing waves}

It is worth noting that although this paper is devoted to traveling wave (TW) accelerators, most of its results can also be applied for standing wave (SW) linacs. The longitudinal oscillations of particles in a traveling wave are described by differential Eqs. (9) and (10), or more generally by those described in [55], the solutions to which define the area of particles capture into the accelerating regime as well as the frequency and attenuation of phase oscillations. In standing wave accelerating structures, the longitudinal (phase) oscillations, in general, are described not by differential equations but finite difference equations [7]. However, if the phase variation of the RF field and energy gain do not change much from cell to cell in an SW structure, then the finite differences can be replaced by differentials, allowing evaluation of the motion in an equivalent TW structure. Numerical estimations [56] demonstrate that this reduction does not lead to any significant errors, especially in high-level accelerator design.

Let us consider the longitudinal component of an axially symmetric standing wave $E_{z}(r, z, t)=E_{0}(r, z) \cos \omega t$. The function of the amplitude distribution in a cylindrical corrugated waveguide can be represented as [57]:

$$
E_{0}(r, z)=E_{0} J_{0}\left(\frac{2 \pi r}{\lambda}\right)+\sum_{m=1}^{\infty} A_{m} I_{0}\left(k_{m} r\right) \cos \frac{2 \pi m}{\lambda} z
$$

where $J_{0}$ and $I_{0}$ are Bessel functions of the first kind and modified Bessel functions, respectively. The instantaneous value of the longitudinal electric field can, therefore, be presented as:

$$
E_{z}(r, z, t)=\sum_{m=-\infty}^{\infty} B_{m}(r) \cos \left[\omega t-\frac{2 \pi m}{\lambda} z\right]
$$

This series is a sum of the field harmonics with amplitudes $B_{m}$ that travel in positive $(m>0)$ and negative $(m<0)$ directions. By applying this expression into Eq. (8), it is possible to calculate the energy gain:

$$
\Delta W=q \int_{L} E_{z}(r, z) \cdot d z=q \sum_{m=-\infty}^{\infty} B_{m}(r) \int_{L} \cos \left[\frac{2 \pi}{L}(k-m) \cdot z+\varphi_{0}\right] d z
$$



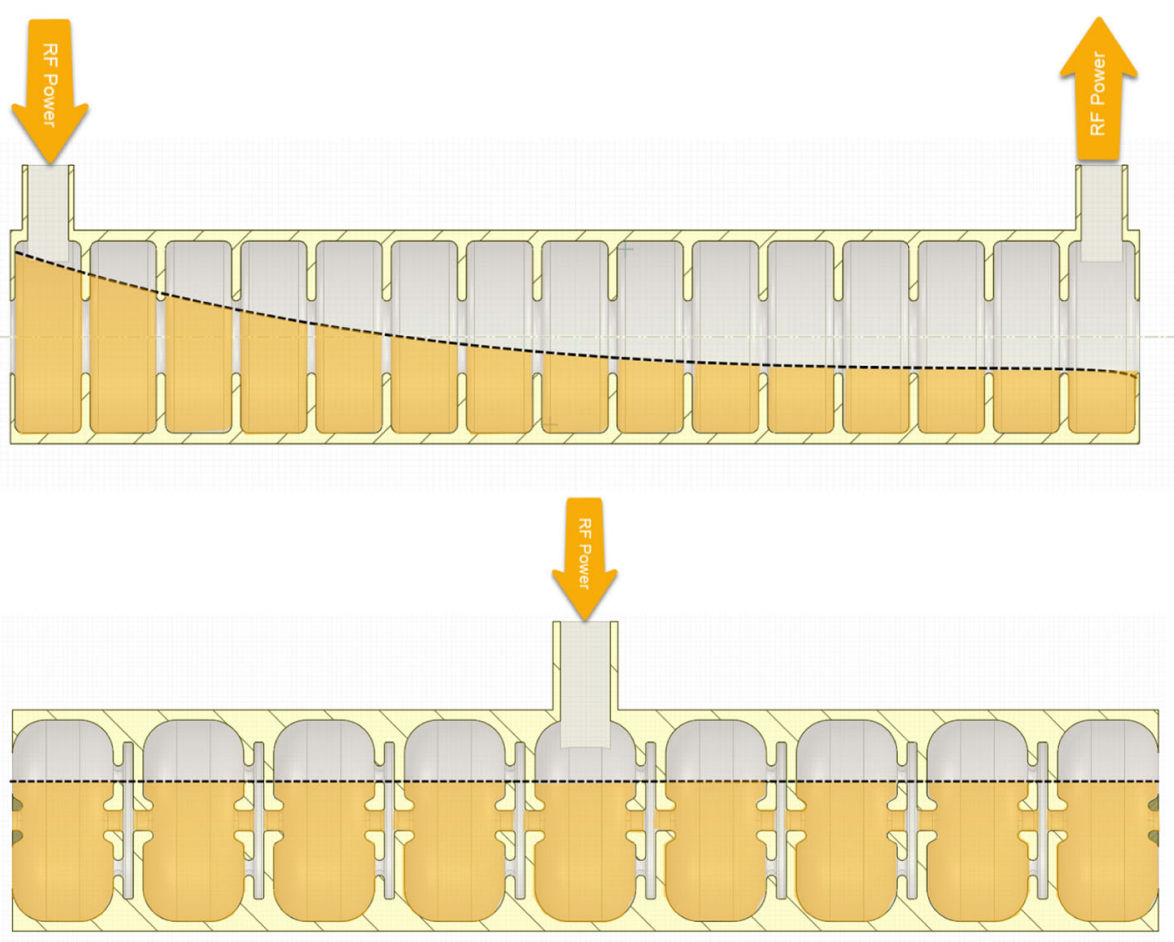

Fig. 15 Power distribution mechanism in TW (top) and SW (bottom) structures

At the same time,

$$
\int_{L} \cos \left[\frac{2 \pi}{L}(k-m) \cdot z+\varphi_{0}\right] d z=\left\{\begin{array}{c}
L \cdot \cos \varphi \text { for } m=k \\
0 \text { for } m \neq k
\end{array}\right.
$$

which means that $\Delta W=q B_{k}(r) \cdot L \cdot \cos \varphi$, and only one traveling harmonic $(m=k)$ transfers the energy from the SW to the beam. This harmonic is called an equivalent traveling wave or accelerating wave and allows using the theory and guidance for the buncher design presented in this paper for both TW and SW accelerators.

Apart from the different modes that are used in SW structures $(\pi$-mode in terms of beam dynamics, usually realized in $\pi / 2$ bi-periodic structures [15]), the other significant difference that affects beam dynamics in properly designed structures is the power propagation (Fig. 15). In TW structures, the EM wave propagates from the power inlet to the outlet while being attenuated along its path, while SW structures are filled with power through a single port, and the power is uniformly distributed among all cells. This difference can play a significant role when high-current beams are accelerated. As was discussed above, an electron beam can change the amplitude of the accelerating EM wave: gradually, from cell to cell in TW structures and uniformly in SW linacs, as shown in Fig. 15. More details about accelerating structures can be found in [58]. 


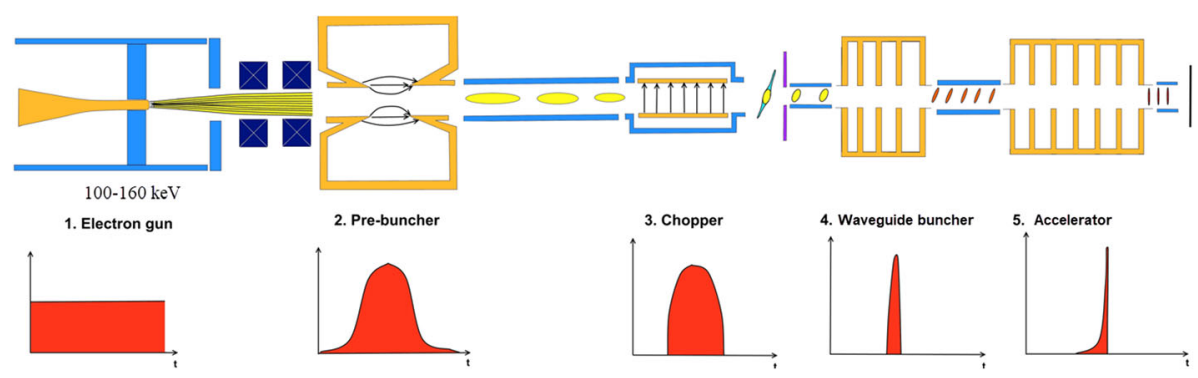

Fig. 16 Typical layout of a high-current (high beam transmission) accelerator [65] (top) and the electron beam longitudinal profile development (bottom)

\section{Buncher types overview and design strategy}

As mentioned in Introduction, a typical industrial electron accelerator, shown in Fig. 5, consists of the DC injector (gun) that accelerates the beam to energies of tens of $\mathrm{keV}$, a bunching ion that groups the DC beam into small bunches that are further accelerated in sections with phase velocity equal to the speed of light. Usually, all these elements are physically combined into a single accelerator assembly for compactness and simplicity of fabrication/operation. Such accelerators are usually designed to provide moderate beam transmission rates on the order of 30-60\% [1, 21, 44, 49]. In high-current/high-power accelerators, where high beam transmission is very important, the layout can be more complex and may include $>100 \mathrm{kV}$ DC gun, separate pre-buncher and buncher and more elements as shown in Fig. 16. The DC beam is injected at high energy, focused and passed through a pre-buncher, providing longitudinal focusing. Then, an RF chopper [59] might be used to cut the beam tails. The chopper is usually a resonator with transverse electric field orientation that deflects particles that are longitudinally offset from the beam center. These particles are then eliminated when the beam passes through a slit collimator. The beam is injected into a separate buncher with a tapered or multi-section velocity waveguide structure, where it is shaped and optimized for injection into the $\beta=1$ structure.

In general, industrial-grade accelerators with multi-MW RF power [60-63] tend to have a shorter 1-3 cell buncher design to trade off the capture efficiency for compactness and simplicity, while high-current and scientific-grade linacs [64] implement a classical "prebuncher-buncher-accelerator" concept to maximize capture.

While the particles injected from a DC electron source have the same energies and their phases are uniformly distributed, only part of them will be captured into the acceleration regime. Captured electrons still occupy a wide range of phases, and therefore their energy gain is different since $\Delta W=q E \cos \varphi$. Therefore, by passing through the same distance along with the accelerator, different electrons will gain different energies. This leads to a large energy spectrum in the accelerated beam, which is not desirable for many applications. To reduce the energy spread, the particles must be grouped into bunches with a small phase spread before entering the accelerating $\beta_{\mathrm{ph}}=1$ section. These bunches are separated in space by one wavelength in the accelerating waveguide $\left(\lambda_{\mathrm{w}}=\beta_{\mathrm{ph}} \cdot \lambda\right)$.

Electron bunching can be accomplished with various types of bunchers or even by direct injection into an accelerating section. The simplest case is to use a stand-alone RF cavity that is physically separated from the accelerator by a drift space. In this case, the particles' velocities are modulated by the cavity's RF field, resulting in density modulation after a drift. This time-of-flight bunching method is usually referred to as "ballistic" or "klystron" 
bunching, since klystrons are based on this principle [66, 67]. This type of buncher is also usually called a "pre-buncher."

The other way is to use an accelerating waveguide section with a constant phase velocity. In this case, the beam is bunched due to the phase motion discussed in previous sections. However, sections with $\beta_{\text {ph }}<1$ cannot accelerate particles to high energy, so the phase velocity should be adjusted accordingly with the beam acceleration. This type of bunchers is usually called "tapered bunchers" or "bunchers with a tapered velocity" (not to be confused with structures with a tapered aperture). In this case, the RF bucket will shrink as the equilibrium phase $\left(\varphi_{\mathrm{s}}\right)$ falls below $\pi / 2$. These bunchers are very efficient since they allow simultaneous acceleration and bunching. As a reference, such a scheme is also applied for continuous wave ion RF quadrupoles (RFQs), where the pre-buncher is a multi-harmonic buncher (MHB) [68] and the tapered buncher is realized inside the RFQ channel. It allows reaching high capture and small longitudinal emittance $[69,70]$.

There are other methods to improve the bunching and reduce the energy spread, such as utilizing an amplitude jump (instantaneous change) or rapidly change the equilibrium RF phase. A radical method is to use a deflecting cavity to cut the beam tails and thus reduce its phase length. These techniques can also be combined to optimize the performance of a particular linac. In each particular case, the choice of a buncher design is defined by several factors, including beam currents, energy spread, accelerator length, complexity costs, etc.

Table 2 presents a brief overview and comparison of the different buncher types and highlights their positive and negative properties. It also provides a snapshot of the typical cases where such bunchers are used. In the following sections, we will discuss the techniques mentioned above in more detail. However, it is worth mentioning that there is no single or universally applicable buncher design that is suitable for all applications. This is especially true for novel applications, or sophisticated beam parameters, such as ultra-clean energy spectrum, ultra-high-current transmission, energy variation, and ultra-low-power operation. In many cases, a new accelerator requires the unique buncher design. In the following sections, we will try to familiarize the reader with the different bunching techniques and provide some handy analytical tools for rapid practical design of complex accelerating systems.

Bunchers can also be classified by their length:

- In long bunchers (>10 cells), the amplitude and phase velocity variation can be made very smooth, allowing fine, almost adiabatic, shaping of the bunch and extremely fine parameters (one can make a comparison with proton/deuteron RFQ accelerators that can provide $>90 \%$ beam transmission, simultaneously with acceleration over long distances [75]). These bunchers have low RF fields and are very friendly to low injection voltages and low-RF power accelerators, which can be regarded as a technological simplification of linac systems. On the other hand, low-energy beams are prone to space charge and RF defocusing effects that might require external focusing systems [76]. Also, as was discussed in Sect. 2.5, TM- low- $\beta$ structures are very inefficient in terms of RF power consumptions, which may be a problem in case of power sources with limited capacity.

- In short bunchers (1-5 cells), the electrons are accelerated very rapidly thanks to the high RF fields, meaning that phase oscillations stop very quickly [77]. On the one hand, this allows a very simple design even with no external focusing [78], but, on the other hand, the beam transmission is usually limited to $30-50 \%[49,63,79]$.

Besides the consideration for beam transmission, it is important to consider engineering issues of the design. For example, each unique cell in a conventional DLS accelerator adds several hours to the RF design, engineering design, programming, setup, machining, quality assurance and tuning steps, which in total can sum up to 40 labor hours per unique cell. 
Table 2 Comparison of different buncher types used in electron linacs

\begin{tabular}{|c|c|c|c|}
\hline Buncher type & Pros & Cons & Typical Application \\
\hline Ballistic buncher & Improves capture & $\begin{array}{l}\text { Requires a separate RF } \\
\text { cavity and sometimes } \\
\text { a separate RF source; } \\
\text { requires a drift space }\end{array}$ & $\begin{array}{l}\text { As a pre-buncher in } \\
\text { high-current } \\
\text { accelerators }\end{array}$ \\
\hline $\begin{array}{l}\text { No buncher (injection } \\
\text { into } \beta \mathrm{ph}=1 \\
\text { structure) }\end{array}$ & Simplest design & $\begin{array}{l}\text { Poor capture, phase and } \\
\text { energy spread; } \\
\text { requires high-voltage } \\
\text { injection }\end{array}$ & $\begin{array}{l}\text { In large facilities with a } \\
\text { high-voltage injection } \\
\text { or in industrial } \\
\text { accelerators } \\
\text { over-simplified for } \\
\text { cost/dimensions } \\
\text { reduction, where } \\
\text { beam transmission is } \\
\text { not important; or in } \\
\text { mm-wave } \\
\text { accelerators [71, } \\
72-74] \text { where } \\
\text { low-beta cavities are } \\
\text { not feasible }\end{array}$ \\
\hline $\begin{array}{l}\text { Waveguide buncher } \\
\text { with matched } \\
\text { constant velocity } \\
(\beta \text { ph }=\beta \text { inj })\end{array}$ & $\begin{array}{l}\text { Simple design, } \\
\text { provides the highest } \\
\text { capture ratio }\end{array}$ & $\begin{array}{l}\text { Sometimes challenging } \\
\text { to realize (need low- } \beta \\
\text { cavities or high } \\
\text { voltage injection or } \\
\text { both); limited energy } \\
\text { gain-requires } \\
\text { further bunching } \\
\text { stages }\end{array}$ & $\begin{array}{l}\text { As a first stage in } \\
\text { multi-section } \\
\text { bunchers, in } \\
\text { high-current } \\
\text { accelerators, where } \\
\text { beam capture is } \\
\text { important }\end{array}$ \\
\hline $\begin{array}{l}\text { Waveguide buncher } \\
\text { with low-energy } \\
\text { injection }(\beta \text { ph }>\beta \text { inj })\end{array}$ & $\begin{array}{l}\text { Simple design; allows } \\
\text { using low-voltage DC } \\
\text { gun or efficient } \\
\text { high-phase-velocity } \\
\text { RF cavities; allows } \\
\text { energy spread } \\
\text { reduction techniques }\end{array}$ & $\begin{array}{l}\text { Moderate bunching } \\
\text { efficiency, high } \\
\text { amplitude } \\
\text { single-section } \\
\text { bunchers can have } \\
\text { dramatic reduction in } \\
\text { capture ratio }\end{array}$ & $\begin{array}{l}\text { In accelerators with } \\
\text { limited injection } \\
\text { energy, or where } \\
\text { beam transmission is } \\
\text { not essential, or in } \\
\text { accelerators with high } \\
\text { RF power (strong RF } \\
\text { fields) }\end{array}$ \\
\hline Multi-section bunchers & $\begin{array}{l}\text { Better capture and } \\
\text { beam parameters than } \\
\text { in a single-section } \\
\text { buncher }\end{array}$ & $\begin{array}{l}\text { More challenging beam } \\
\text { dynamics, RF design } \\
\text { and tuning }\end{array}$ & $\begin{array}{l}\text { This buncher is a } \\
\text { balanced alternative } \\
\text { between single } \\
\text { section and tapered } \\
\text { waveguide bunchers } \\
\text { in terms of } \\
\text { complexity and beam } \\
\text { parameters }\end{array}$ \\
\hline $\begin{array}{l}\text { Tapered (velocity) } \\
\text { bunchers }\end{array}$ & $\begin{array}{l}\text { Can provide the best } \\
\text { capture and beam } \\
\text { parameters along } \\
\text { with simultaneous } \\
\text { acceleration }\end{array}$ & $\begin{array}{l}\text { Most challenging } \\
\text { design }\end{array}$ & $\begin{array}{l}\text { In high-current } \\
\text { accelerators, or when } \\
\text { high transmission is } \\
\text { required, or in } \\
\text { accelerators where } \\
\text { specific beam } \\
\text { parameters are } \\
\text { required (low-energy } \\
\text { spread, } \\
\text { multiple-energies, } \\
\text { energy, current or } \\
\text { other parameters } \\
\text { variation) }\end{array}$ \\
\hline
\end{tabular}


More complex shapes (i.e., cells with drift tubes, inter-cell coupling, etc.) may require extra tooling. Additional features like tuning pins can also increase the final cost of the system. For example, in small (low- $\beta$ or high-frequency) cells, it is not always possible to add tuning to the cell directly due to their size (length), and machining tuning features after braze might be required, which adds considerable time and costs. Therefore, the accelerator physicist who designs the industrial linac (and buncher) must take all these consideration into account and find a compromise between beam parameters and linac complexity.

Finally, I would like to mention that analytical methods for buncher design provide very limited results, and the exact solution to the electron equations of motion in RF fields should be found numerically. The typical process of linac design includes rough analytical estimations, preliminary design using a combination of analytical and numerical methods (i.e., motion equation solution), particle tracking in realistic 2D or 3D fields (Parmela [80], ASTRA [81], GPT [82], TRACK [83], etc.) and, finally, beam dynamics verification in self-consistent codes such as CST Particle Studio (PIC) [84] and Magic3D [85]. The latter step might be optional due to the huge computational resources required for self-consistent $3 \mathrm{D}$ transient simulations.

One of the tools that allow robust beam dynamics analysis in accelerating waveguide bunchers is the Hellweg ${ }^{2}$ code [86] developed by the author of this paper and used in this tutorial for demonstration purposes. However, it is also possible to use approximate solutions for the investigation and design of waveguide bunchers. Hellweg works on Microsoft Windows and includes a graphical user interface (GUI). The input data for this program are a plain text file that specifies the beam (initial phase, energy and phase distributions, input current, Twiss parameters), the traveling wave structure (input power, frequency and cell parameters), and computational parameters (number of macroparticles, mesh, etc.). This allows using external optimization algorithms above Hellweg.

The numerical model is based on self-consistent equations describing electron motion in waveguide structures with variable dimensions [87]. Originally limited to $2 \mathrm{D}$ dynamics in an axisymmetric linac, these equations capture the self-consistent RF-field amplitude created by the beam, with accurate treatment of each particle's phase. Initially, Hellweg used the simplified ellipsoid-based single-bunch space charge model [88] and simple approximations of external magnetic fields. Very recently, RadiaSoft has improved Hellweg as follows: ported to Linux and developed a prototype web-browser GUI [89], generalized the fundamental equations to a fully $3 \mathrm{D}$ representation [55], and significantly improved the generality and robustness of the space charge calculations [90]. The results obtained with Hellweg have been carefully benchmarked against analytical models, other codes, and experimental results [55, 63, 86, 89 91. Hellweg is open source and available on GitHub [92]. In the following sections, we will use this code to illustrate the beam dynamics in the described bunchers.

\subsection{Ballistic bunchers (pre-bunchers)}

The bunch's phase length can be reduced with an RF resonator, followed by a drift space. Although this problem has been solved many times in the klystron theory $[15,66]$, we will approach it from the bunching efficiency and electron linac point of view to obtain some practical expressions. The schematics of a ballistic buncher is presented in Fig. 17. A continuous beam from the DC gun with a voltage $U_{\mathrm{DC}}$ is injected into an RF cavity with a small AC voltage $U(t)=U_{\mathrm{RF}} \cdot \sin \left(\omega_{\mathrm{RF}} t\right)$, operating at a frequency $\omega_{\mathrm{RF}}=2 \pi / T_{\mathrm{RF}}$. Particles that arrive in the accelerating phase gain energy and start to move faster, while the particles

2 The code is available for evaluation from the author. 


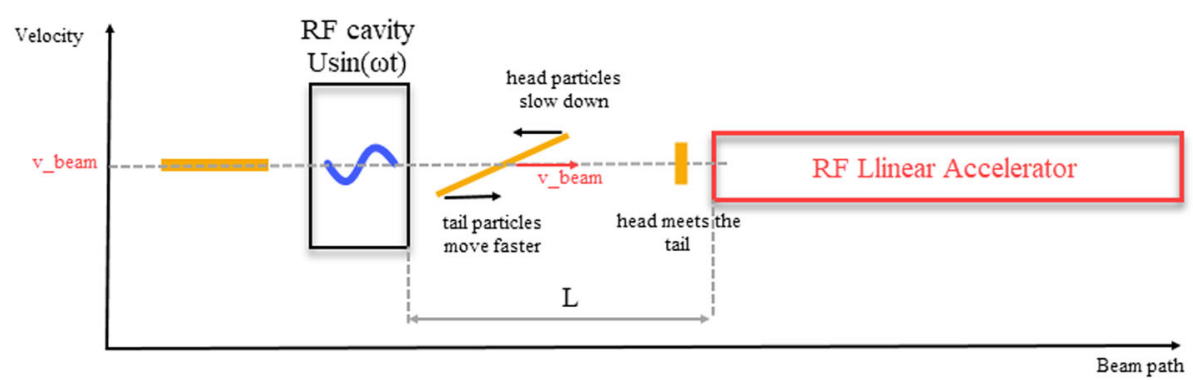

Fig. 17 Principle of ballistic bunching. The DC beam (yellow line) is injected into the RF cavity with a small $\mathrm{AC}$ voltage. Particles that arrived in the accelerating phase gain energy and start to move faster, while the particles that arrived in the decelerating phase slow down. When the beam flies in a space between the RF cavity and accelerator, faster particles meet slower particles and form a bunch

that arrive in the decelerating phase slow down. When the beam flies in a space with a length $\mathrm{L}$, faster particles meet slower ones and the bunch phase length compresses.

The particle's time of flight in the ballistic buncher is calculated in "Appendix" A, where we demonstrate the convenience of introducing the parameter $r$, called the "bunching parameter" [93] that characterizes the pre-buncher:

$$
r=\frac{\pi L}{\beta_{\mathrm{inj}} \lambda} \frac{U_{\mathrm{RF}}}{U_{\mathrm{DC}}}
$$

For a desired phase length or number of particles inside the bunch, it is convenient to use the numerically obtained plot to determine the optimal bunching parameter of the pre-buncher ( $r$ ) [94]. The details can be found in "Appendix" A. By selecting the desired phase length of the bunch, this plot allows estimation the ratio of particles inside this bunch. For example, a 1-rad long bunch will contain $\sim 70 \%$ of the injected particles. This plot also allows us to define the parameter $\mathrm{r}$, required for a practical pre-buncher design. For this case $r=1.8$, and by using the formulae (34), we can find that the drift space length $L$ needs to be $\sim 1.15 \lambda$ for $U_{\mathrm{RF}}=0.2 \mathrm{U}_{\mathrm{DC}}$.

In the example of beam compression in RF, presented in Fig. 18, the phase length of a bunch is $2.1 \mathrm{rad}$ and includes $\sim 80 \%$ of all particles. At the same time, $\sim 92 \%$ of the particles fit into the RF bucket, compared to $68 \%$ calculated by formula (20) for the case when the DC beam has not been pre-bunched. Note that the ballistic buncher does not provide any significant acceleration even to a fraction of the beam due to small RF amplitudes, and the net acceleration is zero. Therefore, ballistic bunchers are usually used not as separate bunchers, but as pre-bunchers to improve capture efficiency in the following waveguide bunchers.

To design an efficient ballistic buncher, it is necessary to know what number of the injected particles can be grouped within the required phase length. By increasing the factor $r$, the size of the bunch (where most of the particles are concentrated) will shrink, and the density of electrons will grow. However, the total number of electrons in the bunch will decrease (Fig. 19).

It is interesting to discuss the pre-buncher voltage choice. To reduce the energy spread at the linac entrance, the resonator field amplitude $U_{\mathrm{RF}}$ should be chosen to be as small as possible. However, low voltages will lead to long drift space requirements, which is undesirable because of space charge repelling forces that can increase the beam radius, leading to radial beam losses. A long drift is also impractical for linac construction. High $\mathrm{RF}$ voltages can lead to significant nonlinearities of the beam phase space due to relativistic 


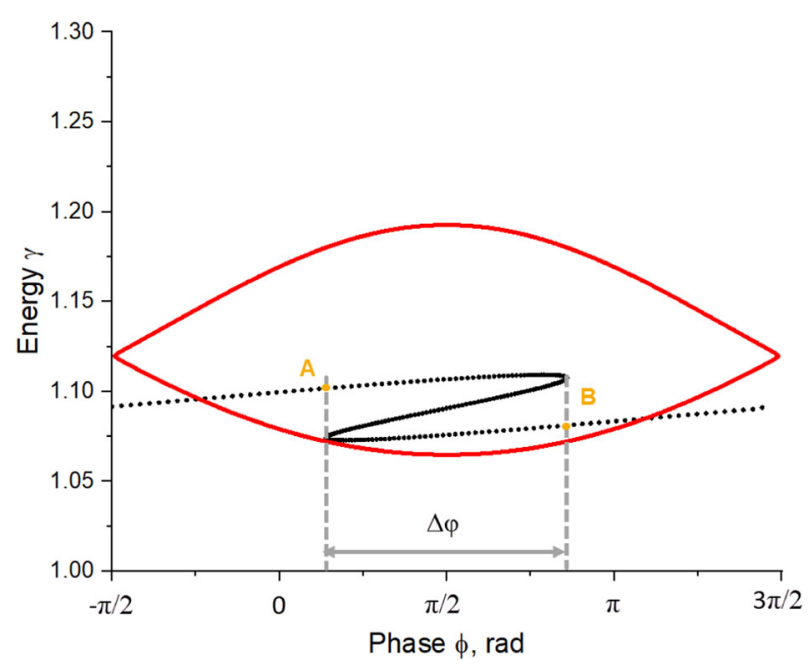

Fig. 18 The beam's phase space after a ballistic buncher with $r=2.5, \beta_{\text {inj }}=0.4$, and $\mathrm{U}_{\mathrm{RF}}=0.2 \mathrm{U}_{\mathrm{DC}}$ (black dots). The interval $\Delta \varphi$ contains $\sim 80 \%$ of all particles, which now can be efficiently accepted by a section with $\beta_{\mathrm{ph}}>\beta_{\text {inj }}$. In this case, $\beta_{\mathrm{ph}}=0.45, A=0.05$ (red-RF bucket), and the net improvement in the capture ratio is $\sim 25 \%$, compared to the case with no pre-buncher

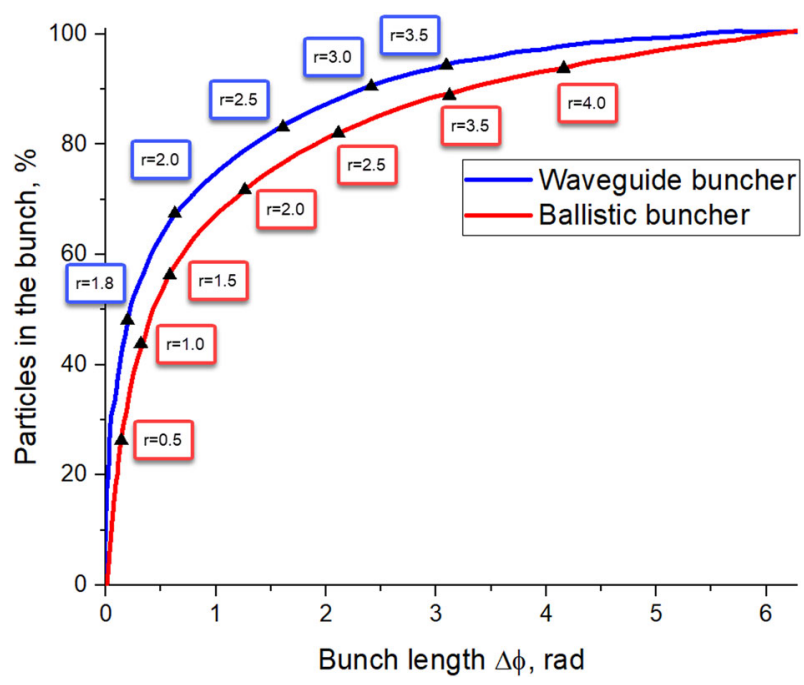

Fig. 19 The number of particles in a bunch with a length $\Delta \varphi$ after ballistic (red) or waveguide (blue) buncher. Black dots represent the values of parameter $r$ for a practical buncher design

effects. In practice, $\mathrm{RF}$ voltages in the range of $(0.2-0.5) \cdot \mathrm{U}_{\mathrm{DC}}$ are recommended, depending on a tradeoff of transmission ratio vs. compactness set by the linac requirements.

The following example will demonstrate how a pre-buncher can improve capture efficiency. In this example, we will consider that a $100 \mathrm{kV}\left(\beta_{\text {inj }} \approx 0.548\right)$ DC electron beam is injected directly into a 70-cm-long S-band (frequency $2856 \mathrm{MHz}$ or $10.5 \mathrm{~cm}$ wavelength) accelerating structure with a constant $10 \mathrm{MV} / \mathrm{m}$ gradient. The normalized field value for this case is $A=\frac{q E \lambda}{W_{0}} \approx 2.05$. Assuming no space charge effects, a 1-mm beam and a strong 
(a)

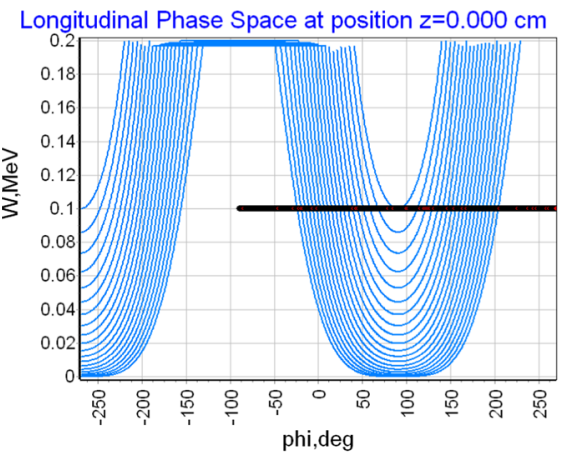

(c)

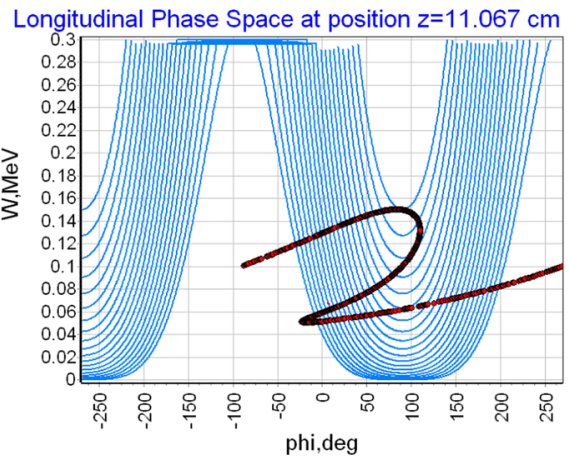

(b)

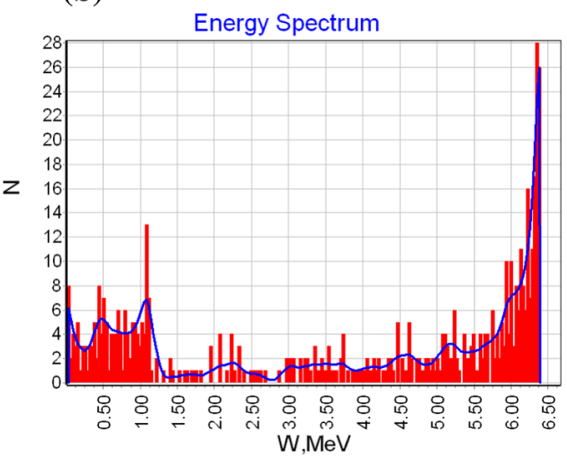

(d)

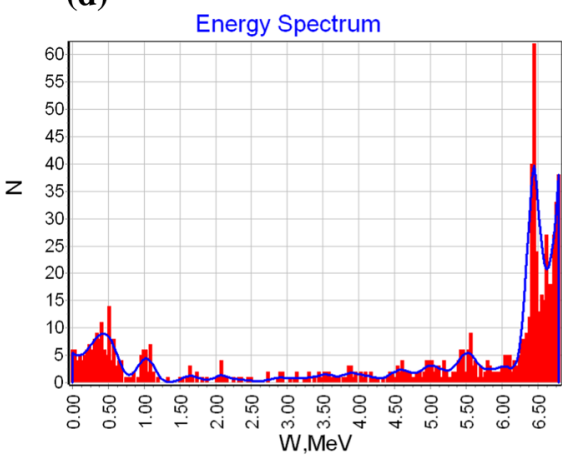

Fig. 20 Phase portraits of 100-keV DC beam, injected directly a into $10 \mathrm{MV} / \mathrm{m} \mathrm{S}$-band accelerating structure and $\mathbf{b}$ after passing through a $50-\mathrm{kV}$ pre-buncher with a $9.15-\mathrm{cm}$ drift space; $\mathbf{c}$ and $\mathbf{d}$ demonstrate beam energy spread after passing through the accelerating structure for cases a) and b), respectively. Simulations performed in Hellweg

solenoid field (1000 Gs) to eliminate transverse beam loss effects, the beam transmission through the structure would be $51.4 \%$, according to Hellweg simulations. If we add a 50-kV pre-buncher, operating at the same frequency and with buncher parameter $r=2.5$ to group $80 \%$ of particles in $1.86 \mathrm{rad}$, similar to Fig. 18, we can estimate the required drift space length as $L=\frac{r \cdot \beta_{\mathrm{inj}} \lambda}{\pi} \frac{U_{\mathrm{DC}}}{U_{\mathrm{RF}}}=9.15 \mathrm{~cm}$. The simulation results for this case demonstrate an improvement in transmission to $77.4 \%$ (Fig. 20).

Although pre-bunchers can substantially improve the beam capture, they also complicate the accelerator design. Due to the low-voltage operation, compared to the accelerating voltage in DLS accelerating structures, and the requirements for a drift space, pre-bunchers are usually implemented as stand-alone cavities with a separate RF coupler. Although they can physically be embedded into the main waveguide in compact accelerator layouts, as shown in Fig. 21, they still need their own RF system. Also, as discussed in the previous section, the drift tube may require external focusing since low-energy beams are prone to space charge repelling forces. For these reasons, pre-bunchers are rarely used in industrial linacs, where compactness, simplicity and cost efficiency are prioritized [1, 4]. However, they are widely used in large high-current machines to improve their beam efficiency. 


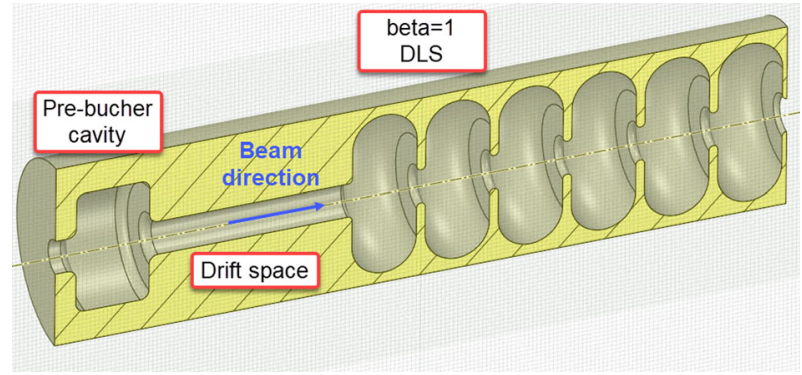

Fig. 21 Illustration of a conceptual design of an S-band pre-buncher with a 9.15-cm drift, followed by a $\beta=$ 1 section, integrated into a single mechanical structure

\subsection{Waveguide bunchers}

The bunching of electrons in an accelerating waveguide is performed due to phase oscillations (particle motion along the phase trajectories) according to the principles described in Sect. 2.1. Due to the shape of the RF bucket, the density of particles will "pulse" during the interaction with the RF wave. By properly adjusting the shape of the bucket, defined by the phase velocity $\beta_{\text {ph }}$ and field amplitude A of the DLS cells, it is possible to achieve high capture efficiency and beam acceleration at the same time.

There are several types of waveguide bunchers used in industrial-grade accelerators that can be classified by the behavior of the $\beta_{\mathrm{ph}}(\mathrm{z})$ and $\mathrm{A}(\mathrm{z})$ functions:

- Bunchers with constant phase velocity (including $\beta_{\mathrm{ph}}=1$ ) that are characterized by moderate phase compression of the DC beam for particles accepted into the acceleration regime, but which at the same time can result in small energy spread as well as design and fabrication simplicity.

- Bunchers with $\beta_{\mathrm{ph}}$ and $\mathrm{A}$ varying smoothly from cell to cell (so-called tapered velocity bunchers), which can provide high capture efficiency due to the more intense phase oscillations that increase energy spread [15]. Depending on the number of cells, these buncher can be very challenging to design, fabricate and tune.

- Bunchers with stair-step functions of phase velocity (so-called multi-section bunchers) comprise the intermediate type between constant and tapered velocity bunchers, and depending on the number of steps, can have a performance closer to either type.

The choice of buncher type is somewhat related to the injection energy and RF power. As a rule of thumb, the lower the injection energy and RF power are, the more complex the bunching section should be. The physical mechanism of phase compression is the same in waveguide bunchers of different types. In the following section, we will provide details and guidance for buncher design, according to these classifications. For bunchers with constant phase velocity, it is possible to derive analytical models to a limited extent. For tapered bunchers, we will present different design approaches. 
(a)

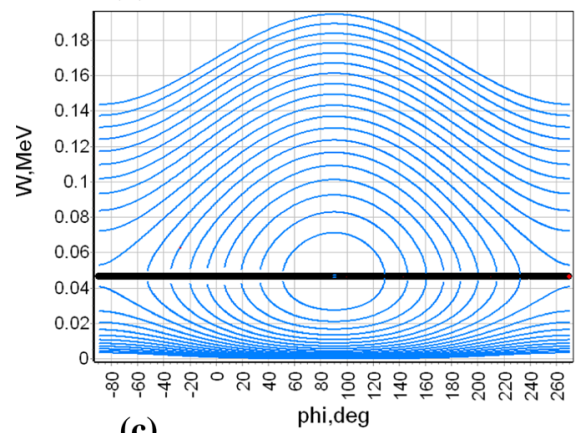

(c)

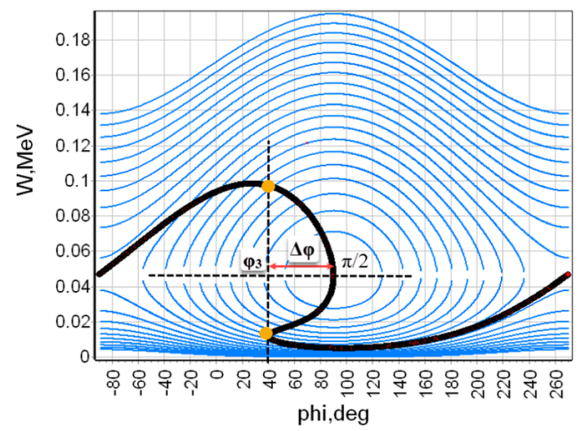

(b)

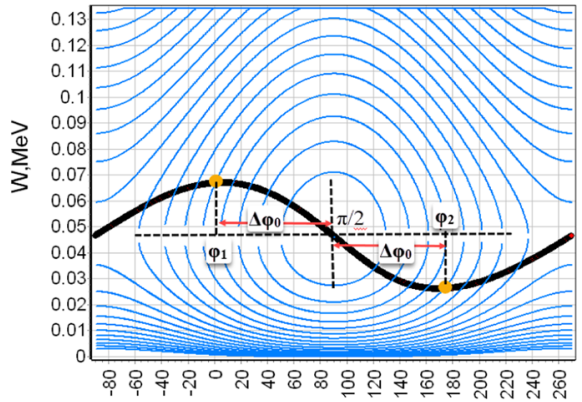

(d)

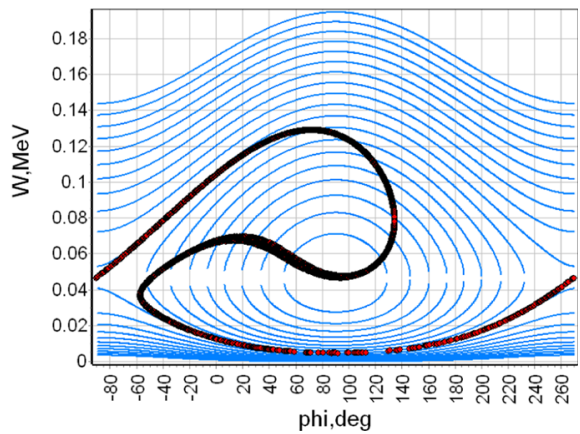

Fig. 22 Phase oscillations of particles in an accelerating waveguide section with $\beta_{\mathrm{ph}}=0.4$, simulated in Hellweg. The beam is injected with $\beta_{\text {inj }}=0.4$. Each plot corresponds to different positions: $\mathbf{a} z=0, \mathbf{b} z=$ $\beta_{\mathrm{ph}} \lambda / 3, \mathbf{c} z=\beta_{\mathrm{ph}} \lambda, \mathbf{d} z=5 \beta_{\mathrm{ph}} \lambda / 3$. It can be seen that oscillation frequencies of particles located on the separatrix and at equilibrium phase $(\pi / 2)$ are equal to zero

\subsection{Waveguide bunchers with a constant phase velocity}

\subsubsection{Bunchers with a phase velocity, matched to the injection energy}

The expression for the frequency of phase oscillations in sections with a constant phase velocity can, as demonstrated in "Appendix" B, found to be:

$$
\Omega=\omega_{\mathrm{RF}} \sqrt{A \frac{\sqrt{\left(1-\beta_{\mathrm{ph}}^{2}\right)^{3}}}{2 \pi \beta_{\mathrm{ph}}} \sin \varphi_{s} \cdot \cos \frac{\psi}{2}}
$$

One type of buncher that we have partially discussed above is an accelerating waveguide section with constant $\beta_{\text {ph }}<1$ chosen to match the injected electrons' velocity. The particles in such a section are completely fit into RF bucket as shown in Fig. 9 and will oscillate around the equilibrium phase as shown in Fig. 11. These plots also demonstrate that, as with a ballistic buncher, the matched velocity buncher does not provide net acceleration to the beam, since the particles will have a sinusoidal energy gain. The distribution of a DC electron beam injected into a waveguide buncher with $\beta_{\text {ph }}=\beta_{\text {inj }}=0.4$ is shown in Fig. 22.

The phase interval that the electrons occupy at the entrance of the buncher (Fig. 22a) will shrink during the motion along the accelerating waveguide (Fig. 22b) until it reaches a minimum value (Fig. 22c) and then starts to expand again (Fig. 22d). As with a ballistic 
buncher, the matched waveguide section can be characterized with a bunching parameter $r$ as demonstrated in "Appendix" C. The numeric values of this parameters can be found in Fig. 19, according to the desired phase length of the bunch and the percentage of particles in this bunch. Then, the required length of the buncher can be found to be:

$$
\frac{L_{b}}{\lambda}=\frac{\beta_{\mathrm{ph}}}{2 \pi}\left(\frac{r}{\Omega}-\varphi_{2}+\varphi_{3}\right)
$$

It is important to highlight that in spite of the similar mechanisms of ballistic and matched waveguide bunchers, the latter has better bunching efficiency, as shown in Fig. 19. For example, a 1-rad bunch can achieve $75 \%$ bunching in a waveguide buncher versus $70 \%$ for a prebuncher. The energy spread after the buncher can be found by using the following expression from "Appendix" C:

$$
\begin{aligned}
& \Delta \beta=\frac{2 \sqrt{\beta_{p h}^{2}+\left(a^{2}-1\right)\left(a^{2}+\beta_{p h}^{2}\right)}}{a^{2}+\beta_{p h}^{2}} \\
& a=\sqrt{1-\beta_{p h}^{2}}+\frac{A \beta_{p h}}{2 \pi}
\end{aligned}
$$

For example, let us consider that we would like to design a buncher with a phase compression ratio of 5. Figure 19 allows us to find the parameter $r=2$. The bunch will contain $66 \%$ of all particles, and the required length of the buncher should be $0.38 \lambda$, which roughly corresponds to 3 cells. The velocity spread will be in the range of 0.17 to $0.58(15-115 \mathrm{keV})$, which corresponds to the numerical simulations shown in Fig. 22c.

This result suggests that to reduce the energy spread in the bunching section, the amplitude of the RF field should be made as small as possible. As with pre-bunchers, this will lead to longer bunching sections, which is undesirable due to the beam transverse divergence effects and low power efficiency of low- $\beta$ structures (see Fig. 14). However, unlike pre-bunchers, waveguide bunchers do not require a drift space and can be integrated into the accelerating structure.

\subsubsection{Low-energy injection}

To avoid lossy DLS waveguide sections with low phase velocities $\left(\beta_{\mathrm{ph}}<0.5\right)$ and/or high voltage DC guns, the beam is often injected into sections with $\beta_{\mathrm{ph}}>\beta_{\text {inj }}$ (we call this case "low-energy injection"). This is a practical approach when the absolute injection energy is low: for example, a $15-\mathrm{kV}$ gun will provide $\beta_{\text {inj }}=0.237$. DLS sections with larger $\beta_{\mathrm{ph}}$ are also easier to fabricate. Here we will use the phase oscillations approach to derive practical expressions for such a buncher design.

When the velocity of the electrons is lower than the phase velocity, the line of the injected particles, plotted on the phase space plane, will lie below the equilibrium energy line, which reduces the capture ratio. Figure 23 demonstrates the beam phase space evolution during bunching in a $\beta_{\mathrm{ph}}>\beta_{\text {inj }}$ section. Particles with $\varphi<\pi / 2$ gain energy, while the particles with $\varphi>\pi / 2$ first decelerate and then accelerate along with the second half of the beam. As can be seen from plot (c), the energy and phase spread at this moment are close to optimal.

To determine the moment when the bunch has minimal phase length, we need to understand that trajectories are not symmetrical across the equilibrium energy line. The analytical expressions for optimal buncher length and corresponding velocity spread can be found in "Appendix" C. However, in practice, the length of such a buncher will depend on the particular design of the accelerator and the beam requirements. For example, if the beam continues its 
(a)

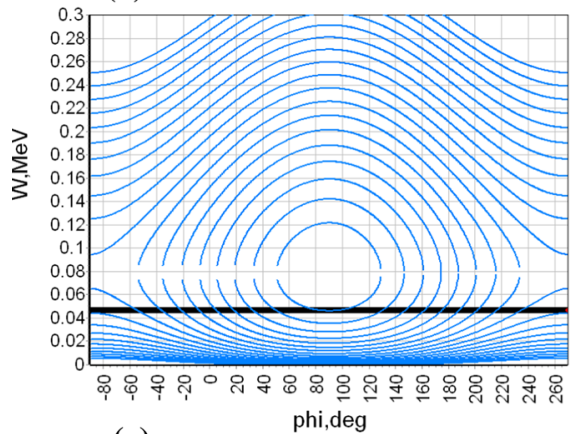

(c)

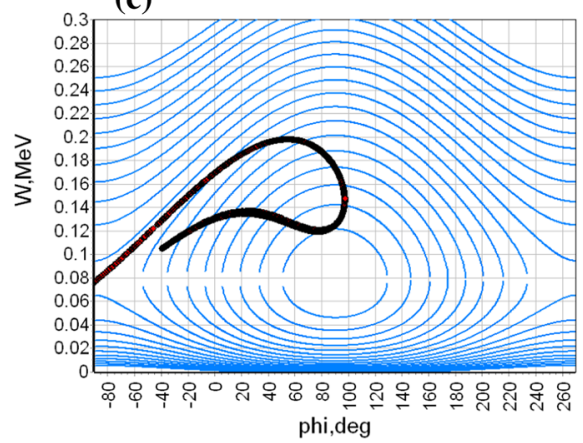

(b)

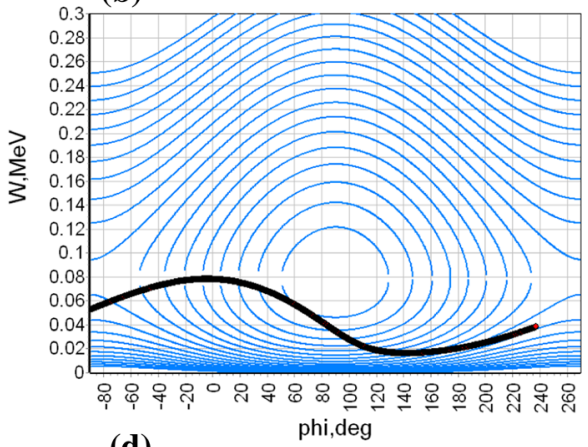

(d)

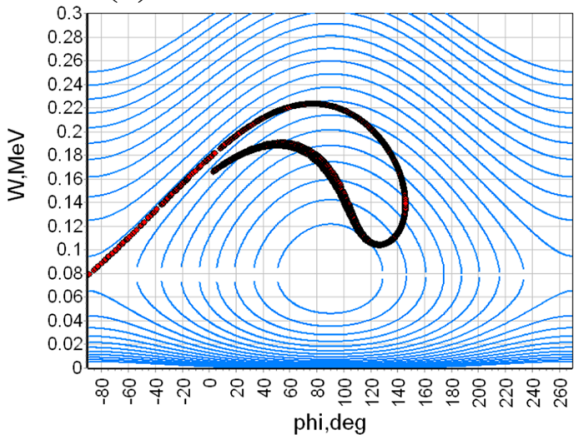

Fig. 23 Phase oscillations of particles in an accelerating waveguide section with $\beta_{\mathrm{ph}}=0.5$, simulated in Hellweg. The beam is injected with $\beta_{\text {inj }}=0.4$. Each plot corresponds to different positions: $\mathbf{a} \mathrm{z}=0, \mathbf{b ~ z}=$ $\beta_{\mathrm{ph}} \lambda / 3, \mathbf{c} \mathrm{z}=4 \beta_{\mathrm{ph}} \lambda / 3, \mathbf{d} z=7 \beta_{\mathrm{ph}} \lambda / 3$. When the beam head passes the $\pi / 2$ phase, it becomes over-bunched, and the head can split into two peaks

motion in such a section, this will lead to a decrease in the beam energy, and at the same time an increase in phase length due to the different frequencies of phase oscillations. Figure 24 shows the beam density distribution along with phases that support the previous statement.

Moreover, once the beam is over-bunched, the density peak starts to split in two peaks, eventually developing into two energy peaks during acceleration. In many applications, this is undesirable, since a particular energy is required. At the same time, despite the more extensive phase/energy spread of the beam "head," the over-bunched beam has fewer particles in its lowenergy "tail" (see Fig. 5 for visualization). Beam tails are typically not useful in applications and are regarded as contamination. The weight of the over-bunching technique's pros and cons should be estimated for the particular linac design.

The results of the over-bunching technique in a waveguide section with low-energy injection can be demonstrated with the example of a linac, developed by the author, for the Advanced Railroad Cargo Inspection System [95], where the elimination of low- and highenergy tails was required by the detector system to allow effective material discrimination [96]. The linac is a 2-9 MeV S-band TW accelerator with $30 \mathrm{keV}$ injection energy $\left(\beta_{\text {inj }}=\right.$ 0.328 ). An early version of this accelerator consisted of a 4-cell constant phase $\beta_{\mathrm{ph}}=0.4$ waveguide buncher with low accelerating field $\left(A=0.26\right.$ or $\frac{E \lambda}{\sqrt{P}}=60 \Omega^{1 / 2}$-optimal parameters, according to analytical formulas), followed by 22-cells $\beta_{\mathrm{ph}}=1$ section as shown in Fig. 25a. The number of DLS cells was chosen so that $150 \mathrm{~mA}$ can reach $9 \mathrm{MeV}$ energy. The simulation results of this linac, shown in Fig. 26a, b demonstrate that although the buncher 


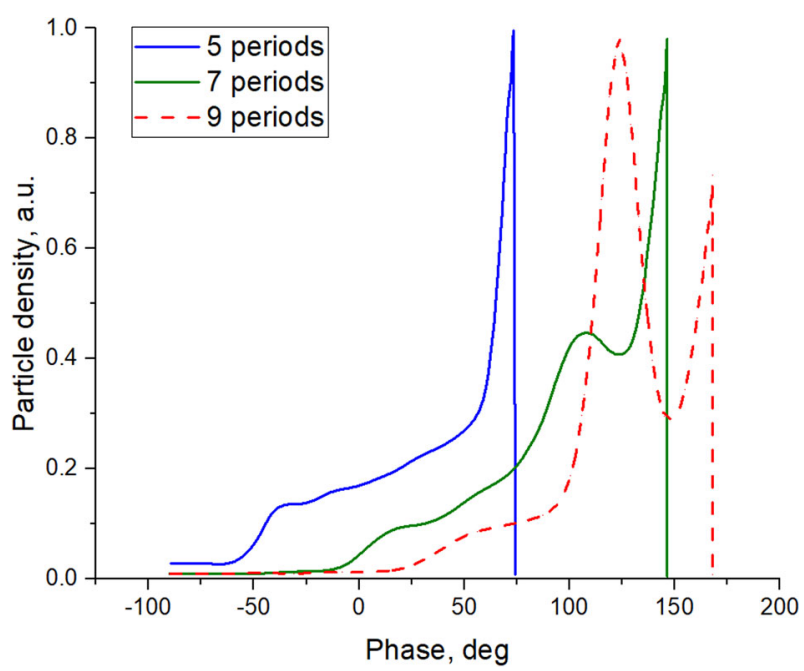

Fig. 24 Phase density (normalized to a maximum for each plot) distribution evolution during bunching: the blue curve corresponds to case (c), and the green to case (d) in Fig. 23. It can be seen that once the beam's head passes across the $\pi / 2$ phase, a second peak starts to develop. These phase peaks can eventually be transformed into energy peaks. At the same time, the low-energy tails become thinner. It should also be noted that the beam has a sharp edge in the head region
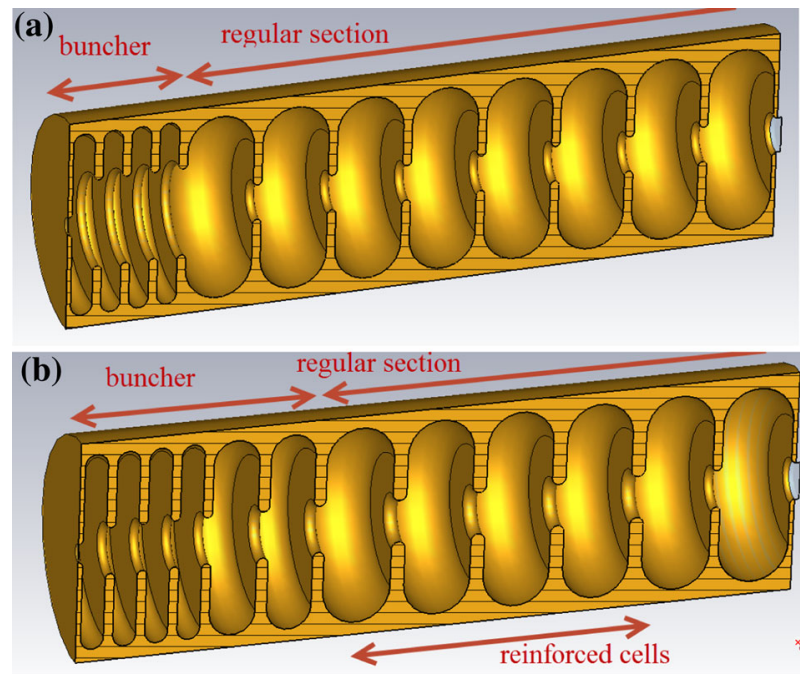

Fig. 25 Two bunching section options for a 9-MeV TW accelerator with reduced energy tails: a Option 1: 4-cell waveguide section with $\beta_{\mathrm{ph}}=0.4$ and $A=0.26$, b Option 2: improved version with $A=1.42$, followed by two $\beta_{\mathrm{ph}}=0.7$ cells

provides good acceptance into the accelerating section, the beam compression is still insufficient to capture most of the particles into the bunch core, leaving them outside of the $\beta_{\mathrm{ph}}=1.0$ bucket. These particles are not lost but form a long low-energy tail.

In order to reduce the number of particles in this tail, we increased the field amplitude in $\beta_{\mathrm{ph}}=0.4$ by factor of 5.4 (from $A=0.26$ to 1.42 ), which resulted in a significantly 

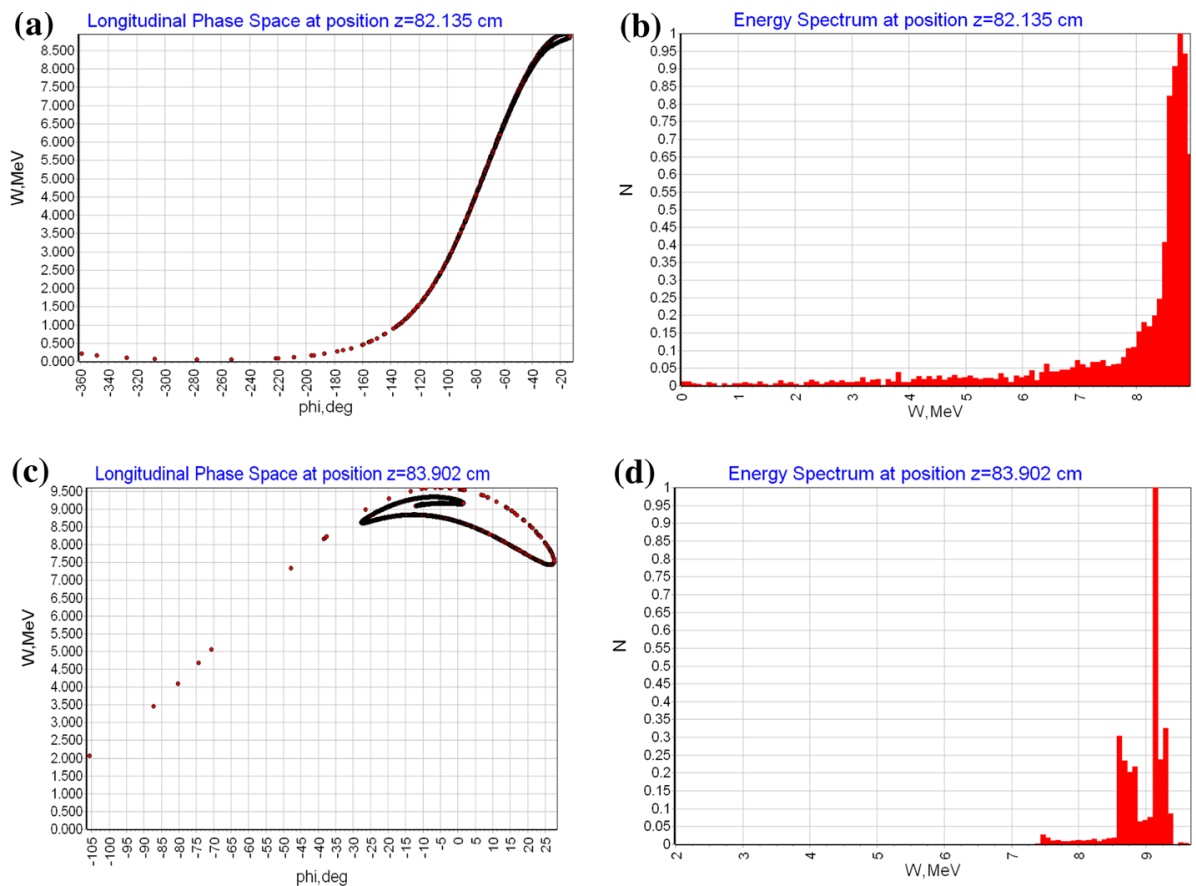

Fig. 26 Phase portraits (a and $\mathbf{c}$ ) and energy spectra (b and $\mathbf{d}$ ) at the end of a 9-MeV TW linac with the buncher options $1(\mathbf{a}, \mathbf{b})$ and 2 (c, d) as referred to in Fig. 25, simulated in Hellweg. The beam passing through long waveguide buncher sections sustains extensive phase oscillations and "folds" in phase space multiple times, "packing" more particles within the bunch core, which would otherwise become a low-energy tail

better phase compression due to the more intensive phase oscillations (the particles now fill the whole RF bucket) at the cost of larger energy spread. We also added two intermediate cells with $\beta_{\mathrm{ph}}=0.7$ to improve the acceptance into the $\beta_{\mathrm{ph}}=1.0$ section (see Fig. 25b) and eliminate the particles outside the RF bucket. As shown in Fig. $26 \mathrm{c}$, d, this technique significantly reduced the number of particles in the low-energy tail. A comparison of beam parameters achieved by both bunchers is provided in Table 3 and demonstrates the parameter trade-offs (transmission, energy spread, beam tails, complexity) that should be considered during the buncher design.

Finally, let us discuss how, in general, the parameters of the buncher influence the beam parameters. Reducing the field amplitude leads to compression of phase trajectories in the vertical direction (according, for example, to (14)) and smaller energy spread. At the same time beam capture will also be lower for the same injection energy $\left(\beta_{\text {inj }}<\beta_{\text {ph }}\right.$ ), according to (20). Higher amplitude bunchers yield larger energy spreads. A similar result is observed by varying the phase velocity. Although the capture is reduced for higher phase velocities, the beam energy grows, and at the same time, the phase length of the beam becomes shorter. The maximum reasonable $\beta_{\mathrm{ph}}$ is set by the acceptable capture ratio, which is not practical to make much less than at least $20-30 \%$.

\subsubsection{Injection of a DC beam into an accelerating section}

A special case of a waveguide buncher with a constant phase velocity is the case where a DC beam is injected directly into a $\beta_{\mathrm{ph}}=1$ section without prior bunching. This solution, which 
Table 3 Comparison of beam parameters at the end of a $9 \mathrm{MeV}$ linac with buncher options 1 and 2 as presented in Fig. 25

\begin{tabular}{lll}
\hline Buncher option & Figure 25a & Figure 25b \\
\hline Buncher type & $\begin{array}{c}\text { Constant phase velocity with } \\
\text { optimal focusing }\end{array}$ & $\begin{array}{c}\text { Constant phase velocity with } \\
\text { over-focusing }\end{array}$ \\
Phase velocity & $\begin{array}{l}4 \times \beta_{\mathrm{ph}}=0.4 \text { cells } \\
4 \times \beta_{\mathrm{ph}}=0.4+2 \times \beta_{\mathrm{ph}}=0.7 \\
\text { cells }\end{array}$ & $\begin{array}{c}1.42\left(\beta_{\mathrm{ph}}=0.4\right) \text {, followed by } \\
2.06\left(\beta_{\mathrm{ph}}=0.7\right)\end{array}$ \\
Field amplitudes A & $0.26\left(\beta_{\mathrm{ph}}=0.4\right)$ & 9.0 \\
Average energy, MeV & 7.8 & 9.3 \\
Peak energy, MeV & 9.0 & 64.8 \\
Beam transmission ${ }^{\mathrm{b}}, \%$ & 66.0 & 0.7 \\
Energy spectrum FWHM, MeV & 0.4 & \\
Low energy tail, $\%$ & 29.9 (below $8.0 \mathrm{MeV})$ & 7.4 (below $8.6 \mathrm{MeV})$ \\
& 46.9 (below $8.5 \mathrm{MeV})$ & 0.36 (above $9.3 \mathrm{MeV})$ \\
\hline
\end{tabular}

${ }^{\mathrm{a}} A=3.15$ for $\beta_{\mathrm{ph}}=1$ section.

${ }^{\mathrm{b}}$ Including transverse losses.

is the easiest to handle theoretically, is the least used [97]. Since no closed phase trajectories exist in $\beta_{\text {ph }}=1.0$ sections (see Sect. 2.2), the particles do not sustain phase oscillation and the capture process occurs as the injected beam moves up to the crest, which leads to beam bunching due to velocity modulation caused by particles experiencing different accelerating field values. In other words, if particles are injected into a structure whose phase velocity equals the velocity of light, they slip in phase, but if the accelerating field is chosen correctly, the particles will asymptotically approach the crest where they can be efficiently accelerated to high energies [10].

To estimate this structure's bunching efficiency, it is convenient to plot its phase trajectories, as shown in Fig. 27. As we previously discussed, the phase oscillations stop when the electron's velocity $\beta$ approaches 1 , and the ultra-relativistic particles have only an insignificant slide along with the wave. This sliding becomes slower as the difference between the beam velocity and phase velocity decreases. Therefore, the phase of all electrons captured in the $\beta_{\mathrm{ph}}=1$ section will gradually decrease during acceleration and approaches an asymptotic value.

The electrons can be captured in a section with a given $\mathrm{A}$ and $\beta_{\mathrm{ph}}=1$ only if their energy is higher than the threshold value, according to Eq. (22):

$$
\beta_{\min }=\frac{\pi^{2}-A^{2}}{\pi^{2}+A^{2}}
$$

Injected electrons are grouped into a phase region from $-\pi / 2$ and $\psi$, which corresponds to the amplitude of phase oscillations for the electron that entered the section in $+\pi / 2$ phase. This value can be found with the help of Eq. (98):

$$
\psi=a \cos \left[1-\frac{2 \pi}{A} \sqrt{\frac{1-\beta}{1+\beta}}\right]
$$


(a)

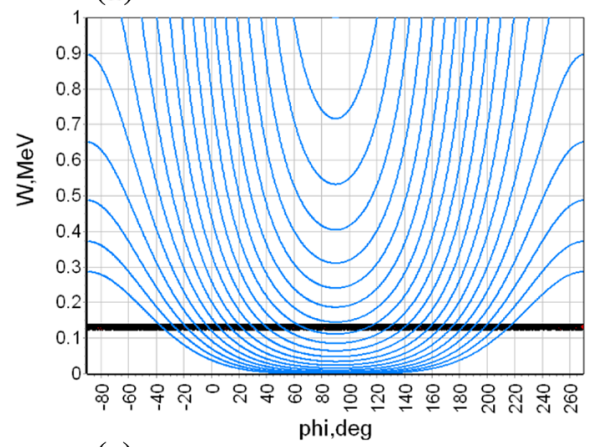

(c)

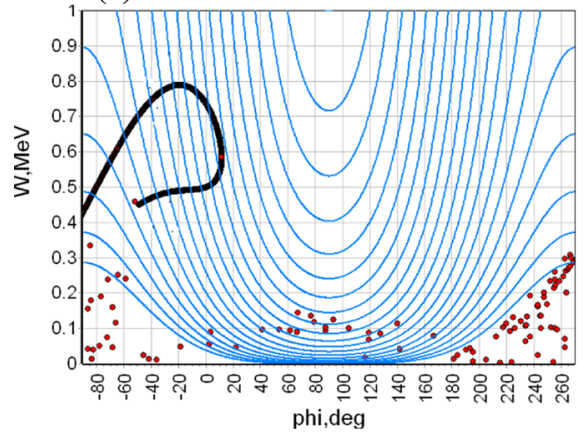

(b)

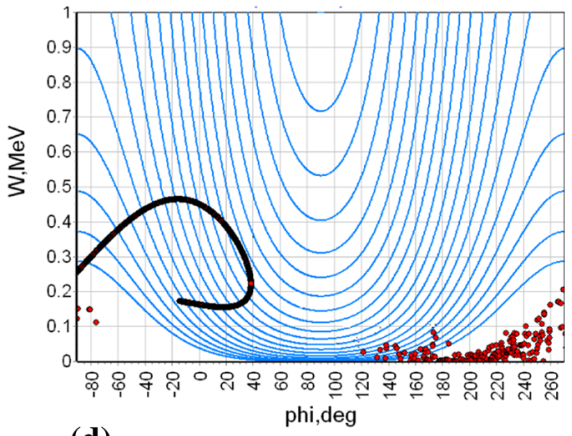

(d)

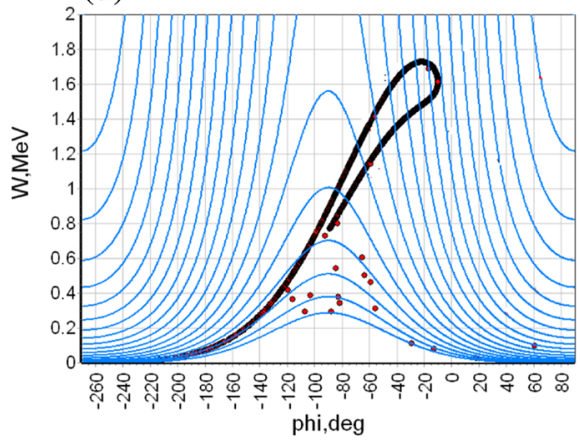

Fig. 27 Phase oscillations of particle, injected at $130 \mathrm{keV}$ into an accelerating waveguide section with $\beta_{\text {ph }}=$ 1 and $A=2$, simulated in Hellweg. Each plot corresponds to different lengths: $\mathbf{a} L=0, \mathbf{b} L=\beta_{\mathrm{ph}} \lambda / 3, \mathbf{c} L=$ $2 \beta_{\mathrm{ph}} \lambda / 3, \mathbf{d} L=4 \beta_{\mathrm{ph}} \lambda / 3$. The last plot is expanded into $-3 \pi / 2$ region to demonstrate the long low-energy tail

For example, for a DC beam injected with an energy of $130 \mathrm{keV}(\beta \sim 0.6)$ into a $\beta_{\text {ph }}=1$ section with $A=2$, approximately $50 \%$ of the electrons can be captured. However, despite the large capture, such a beam will have a significant phase length, and therefore, energy spread (low-energy bunch tail). A compromise solution can be a capture of $\sim 20 \%$. In this case, the phase length of the accelerated bunch will not exceed $0.65 \mathrm{rad}$. This, however, is not a very efficient design in terms of beam transmission.

Based on these considerations, it is possible to conclude that although a DC beam can be directly injected into sections with $\beta_{\mathrm{ph}}=1$, the injection energy must be high enough (>100-150 keV [98]) to achieve reasonable capture. The bunching in such structures is not very efficient because of the large energy spread. The accelerating efficiency in such a structure also deteriorates with this bunching technique, since the bunch is located behind the crest of the EM wave, where its amplitude scales as $\cos \varphi$ and the electrons will continue to slide back in phase.

Therefore, $\beta_{\mathrm{ph}}=1$ bunchers are rarely used as is and often are accompanied by either a pre-buncher, or a $1 / 2$-cell buncher (equivalent to a single cell $\beta_{\mathrm{ph}}=0.4$ buncher with a high field amplitude) or by a phase jump technique to move the bunch head back on the crest. This can be realized by inserting a drift space or a short section with $\beta_{\mathrm{ph}}<\beta$, as will be discussed in the following sections. Another case when $\beta_{\mathrm{ph}}=1$ injection maybe appropriate to use is when it is technically challenging to fabricate lower-beta DLS structures. One example of such a case is a mm-wave accelerator operating at $>100 \mathrm{GHz}$ frequencies [71], shown in 
Fig. 28 Photo of RadiaBeam beam-driven $182 \mathrm{GHz}$ TW structure
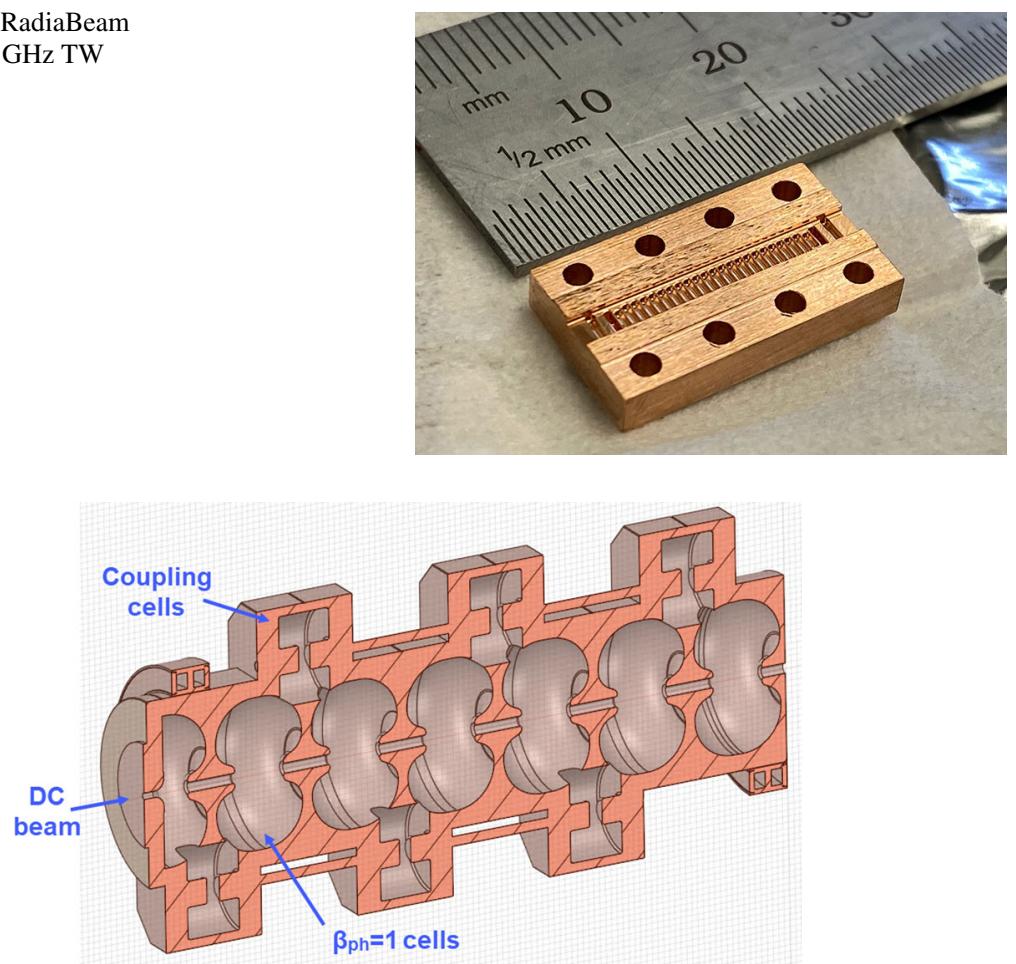

Fig. 29 Example of a typical S-band standing wave side-coupled medical linac with a $1 / 2$-cell buncher

Fig. 28. Such a structure requires fabrication of sub-mm features with micron-level accuracy, so adding lower-beta cells can increase the complexity to an unreasonable level. At present, mm-wave technologies are not ready to be used in industrial accelerators, but might be available in the future [4].

One example of a $\beta_{\mathrm{ph}}=1$ injector was already described in Sect. 3.1, where it was compared for cases with and without a pre-buncher. A similar system was also used in the SLAC Mark III accelerator with injection from an $80 \mathrm{keV}$ DC gun and a pre-bunching cavity [99]. Another example of a $\beta_{\mathrm{ph}}=1$ injector accompanied by a $1 / 2$-bunching cell with the same field amplitude is an S-band accelerating structure design by E. Tanabe [100], which is widely used in medical systems (Fig. 29). While this is a simple design to fabricate, it has a very limited $(<30 \%)$ beam transmission [44], depending on the RF power supply [101] and operating frequency [102] as shown in Table 4.

\subsubsection{Multi-section bunchers}

Since a buncher with constant phase velocity can provide acceleration only to limited energy, the use of a single buncher section might not be sufficient to provide good injection into the accelerating $\left(\beta_{\mathrm{ph}}=1\right)$ section for the reasons described in the previous sections. In this case, multiple sections with gradually increased (from section to section) phase velocities can be used to provide better matching (i.e., larger capture and smaller energy spread) into the $\beta_{\mathrm{ph}}=1$ waveguide. 
Table 4 Beam parameters of Sand X-band versions of a medical SW side-coupled linac consisting of a $\beta_{\mathrm{ph}}=1$ structure with $1 / 2$-bunching cell, simulated in Parmela, including transverse effects

\begin{tabular}{lll}
\hline Frequency, MHz & 2998 & 9300 \\
Number of cells & 6.5 & 13.5 \\
RF power, MW & 2.7 & 1.9 \\
Injection energy, keV & 17 & 15 \\
Beam energy (peak), MeV & 7.42 & 5.30 \\
Beam energy (mean), MeV & 6.50 & 3.60 \\
Beam current, mA & 100 & 35 \\
Field amplitude, MV/m & 56 & 60 \\
Beam transmission, \% & 29.7 & 30.0 \\
\hline
\end{tabular}

The typical strategy for a buncher design with multiple sections of constant phase velocity (both matched and low-energy injection) consists of the following steps, illustrated in Fig. 30: I-choose the combination of phase velocity and field amplitude $\left(\beta_{1}, A_{1}\right)$ so that enough particles are placed within the RF bucket. II-choose the number of cells to compress the beam within the $[-\pi / 2, \pi / 2]$ range. Over-bunching can be acceptable to capture more particles in the beam head at the cost of a broader energy spectrum. III-inject the bunched beam into the next section with a phase velocity higher than the bunch's velocity and field amplitude high enough to fit the bunched beam within the new RF bucket $\left(\beta_{1}, A_{1}\right)$. IV-the length of the second section should be chosen, following a similar approach as step (II). This process should be repeated until the beam is injected into the section with $\beta_{\mathrm{ph}}=1$. The buncher will represent a group of cells with different phase velocities, and its length will depend on the available RF power and desired beam parameters.

In Sect. 3.3.2, we have already showed how the addition of a section with intermediate velocity $\left(\beta_{\mathrm{ph}}=0.7\right)$ between $\beta_{\mathrm{ph}}=0.4$ and $\beta_{\mathrm{ph}}=1$ sections can improve the quality of the beam (i.e., reduce the energy spread and low-energy tails). Another example of when a multi-section buncher design is suitable is a sub-MeV $(180 \mathrm{keV}) \mathrm{Ku}-\mathrm{Band}(16.4 \mathrm{GHz})$ handportable linac for Co-57 radioisotope replacement [103]. The requirements for ultra-small dimensions $(\sim 20 \times 20 \times 10 \mathrm{~cm})$ led to the reduction of all sub-components, the including accelerating structure and RF source (magnetron), which drove the low gun voltage $(15 \mathrm{kV})$, higher frequency and lower RF power choice. With limited available power $(50 \mathrm{~kW})$, the field amplitudes will be very limited: for example, according to Fig. $14 \mathrm{~b}$, a $\beta_{\mathrm{ph}}=0.4$ cell will have $A=0.2$ for apertures $a=0.08 \lambda$, as typically used in DLS structures [58]. Small field amplitudes result in small RF bucket areas and velocity acceptance (see Eq. (14) and (37)), which means that low-energy injection is very limited. In fact, the amplitude threshold for a $\beta_{\text {ph }}=0.4$ cell is $A=0.65$. Therefore, only a long buncher approach (see Sect. 3 introduction) can be applicable. Moreover, at $180 \mathrm{keV}$ beam will have $\beta_{\mathrm{ph}}=0.673$, so such a linac will never have a $\beta_{\mathrm{ph}}=1$ section and will consist only of a bunching section.

The design of the $180 \mathrm{keV} \mathrm{Ku-band} \mathrm{linac} \mathrm{is} \mathrm{presented} \mathrm{in} \mathrm{Fig.} \mathrm{31.} \mathrm{It} \mathrm{consists} \mathrm{of} \mathrm{four}$ waveguide section with different constant velocities. The first section consists of 6 cells with $\beta_{\mathrm{ph}}=0.3$, followed by 8 cells with $\beta_{\mathrm{ph}}=0.5$ and then 4 cells with $\beta_{\mathrm{ph}}=0.6$ and 2 cells with $\beta_{\mathrm{ph}}=0.7$ cells. This design is only $5.9 \mathrm{~cm}$ long, but provides acceleration of a $2 \mathrm{~mA}$ beam from 15 to $180 \mathrm{keV}$ and has a transmission of $25 \%$ without an external focusing system. The phase space evolution of the beam in this linac is presented in Fig. 32 and illustrates the design philosophy described in the beginning of this section: the length of each section is chosen such that the bunch makes $\sim 1 / 4-1 / 2$ of the oscillation period and reaches its maximum energy in a given section; then it enters the next section with a higher phase velocity and the 

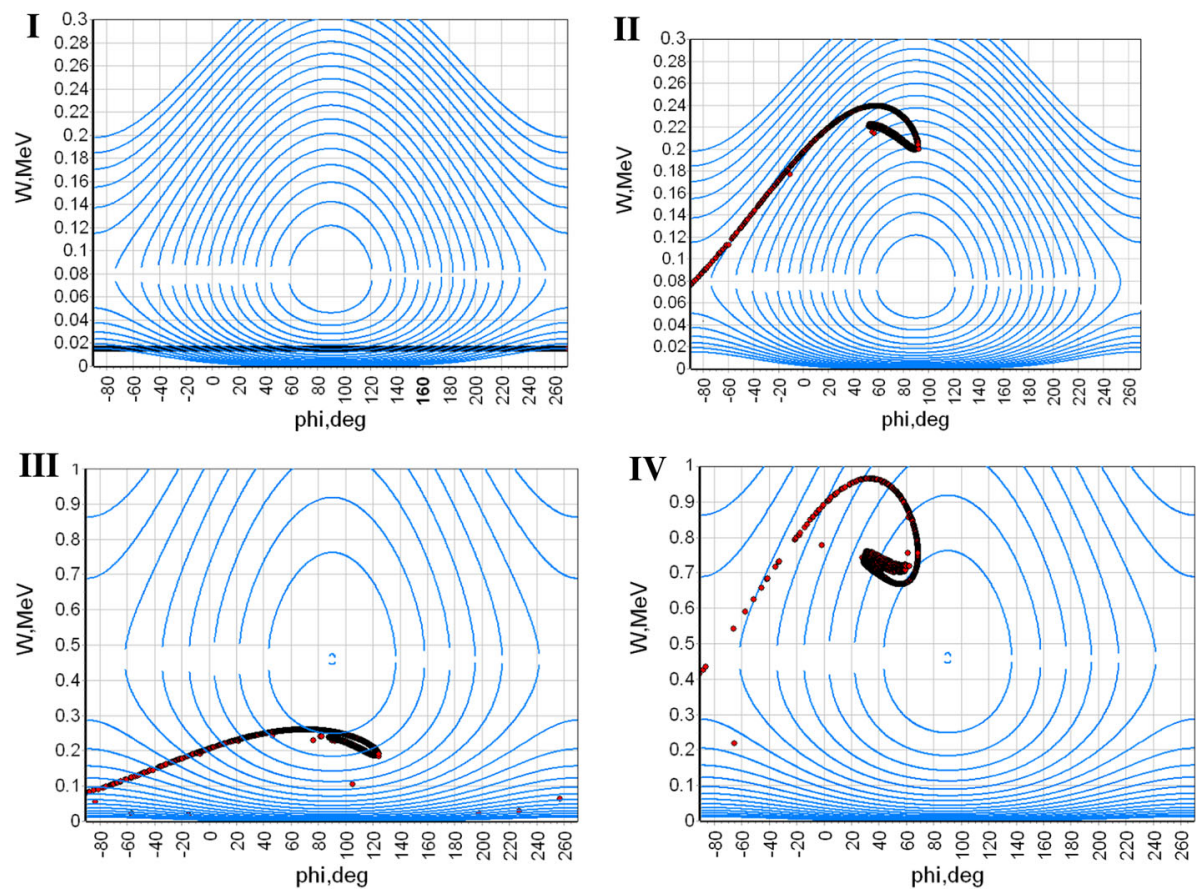

Fig. 30 Evolution of the beam's phase space in a two-section $\beta_{\mathrm{ph}}=$ const buncher, simulated in Hellweg. $\mathrm{I}-15 \mathrm{keV}(\beta=0.237)$ DC beam is injected into $\beta_{\text {inj }}=0.5$ section. II-After passing six cells, the bunch is formed. III-the second section has a phase velocity of $\beta_{\mathrm{ph}}=0.9$. IV-The beam is accelerated to ultrarelativistic energies, and the shaped bunch is ready for injection into the $\beta_{\mathrm{ph}}=1$ section. The low-energy tail is almost eliminated

process repeats until the beam reaches the $\beta_{\mathrm{ph}}=1.0$ section or the required energy as in the present example. The parameters of the accelerator are summarized in Table 5.

In general, the multi-section buncher design is an intermediate step between bunchers with a constant phase velocity where all cells are identical, and with tapered phased velocity where each cell has unique parameters. Unlike the latter, multi-section bunchers are easier to design, since it is intuitively clear how to choose the length, field amplitude and phase velocity of each section just by looking at the beam's phase space development. If some special parameters are required (for example, energy spread reduction or beam tail elimination), it is also possible to apply overfocusing or the phase/amplitude jump techniques with visual control of the beam phase space. At the same time, the beam parameters achieved by this buncher type will depend on the designer's skills and diligence and might not be satisfactory for a particular application. In that case, the design of a more gentle (and more complex) buncher might be required.

\subsection{Waveguide bunchers with tapered phase velocity}

Although waveguide bunchers with constant phase velocity are very appealing due to their simplicity, bunchers where phase velocity and field amplitude change from cell to cell are somewhat more popular since they can provide better efficiency [15, 23, 107]. Such bunchers are usually referred to as waveguides with a tapered phase velocity. They can ultimately be 


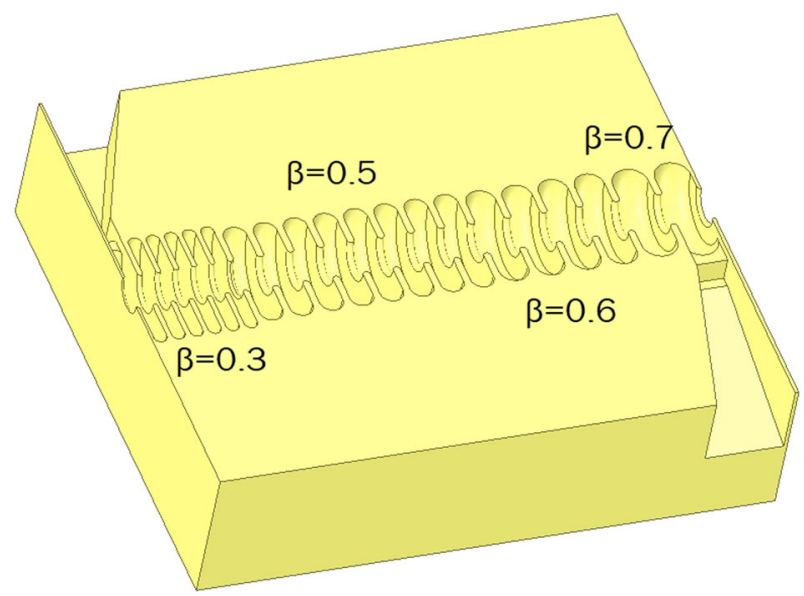

Fig. 31 Example of a buncher for a 40-kW $16.4 \mathrm{GHz}$ linac, consisting of four $\beta_{\mathrm{ph}}=$ const sections: six cells with $\beta_{\mathrm{ph}}=0.3$, eight cells with $\beta_{\mathrm{ph}}=0.5$, four cells with $\beta_{\mathrm{ph}}=0.6$, and two cells with $\beta_{\mathrm{ph}}=0.7$. The available power restricts the length due to low field amplitude A

considered as multiple waveguide sections, each consisting of a single cell, so that the methods from the previous chapters can be applied. However, such an approach is not very efficient since it requires to solve a problem with many variables (at least two for each unique cell). Therefore, it is convenient to reduce this problem to some general solution with the minimum number of variables.

When $\beta_{\mathrm{ph}}(\mathrm{z}) \neq$ const, the equilibrium phase is not equal to $\pi / 2$, since the velocity of synchronous particle must also increase, which is only possible in the accelerating phase. Let us consider the motion in such a section assuming that the field amplitude is constant $(\mathrm{A}(\mathrm{z})=\mathrm{const})$. The equilibrium particle will always travel synchronously with the wave at a phase $\varphi_{\mathrm{s}}$. Then, Eqs. (9) and (10) will take the form:

$$
\begin{gathered}
\frac{d \gamma_{s}}{d \zeta}=A \cos \varphi_{s} \\
\frac{d \varphi_{s}}{d \zeta}=0
\end{gathered}
$$

By integrating the first equation and using the second as a boundary condition, we can obtain an important relationship for the phase velocity $\beta_{\mathrm{ph}}(\zeta)=\beta_{\mathrm{s}}(\zeta)$, which can be written in the form [24]:

$$
\beta_{\mathrm{ph}}(\zeta)=\sqrt{1-\left(\frac{1}{\gamma_{0}+A \zeta \cos \varphi_{s}}\right)^{2}}
$$

Unfortunately, in order to obtain the energy and the phase of the other particles at any given point $\zeta$, it is necessary to solve the equations of motion (9) and (10) with the particular initial conditions and the function $\beta_{\mathrm{ph}}(\zeta)$, defined above. The captured electrons will oscillate around the equilibrium phase with oscillation amplitudes that decay with $\zeta$, which leads to a bunching effect.

Electron bunching in a section with tapered phase velocity depends on the initial energy, field amplitude, and equilibrium phase. The oscillations are most intense in the initial part of the buncher, where the particles' velocities are relatively low. By reducing the injection 


\section{(a)}

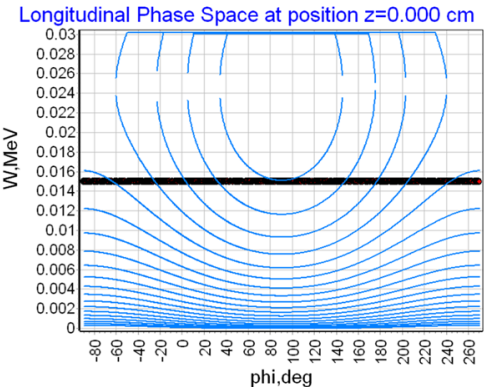

(c)

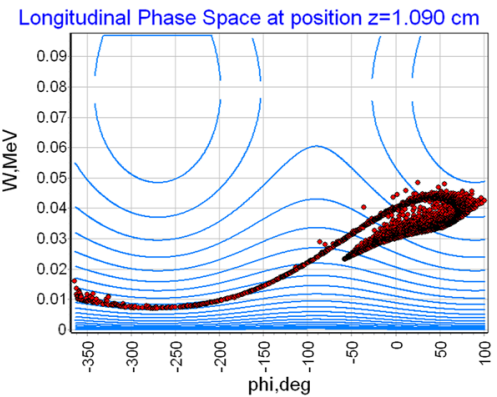

(e)

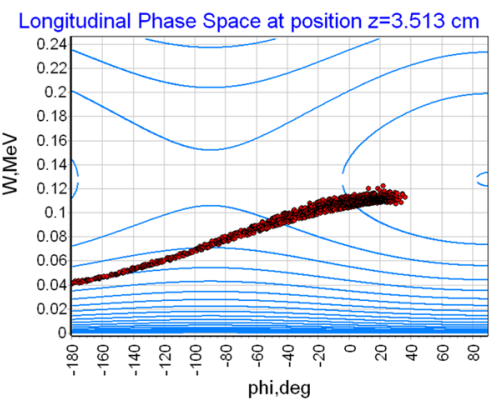

(g)

Longitudinal Phase Space at position $z=4.987 \mathrm{~cm}$

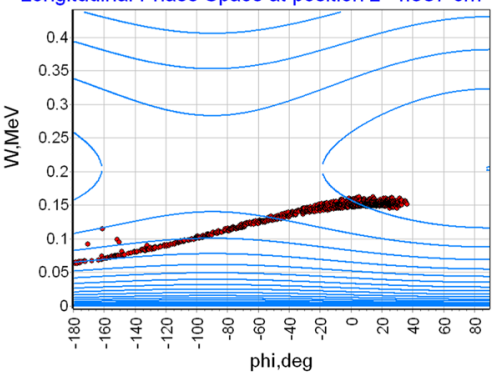

(b)

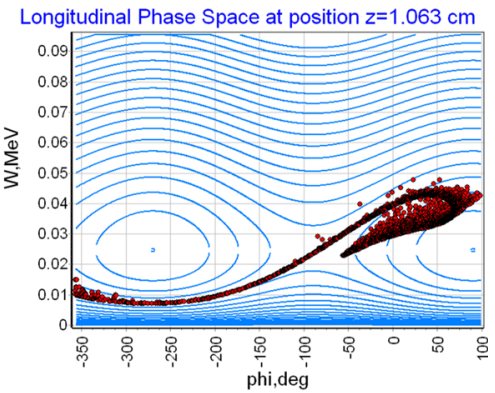

(d)

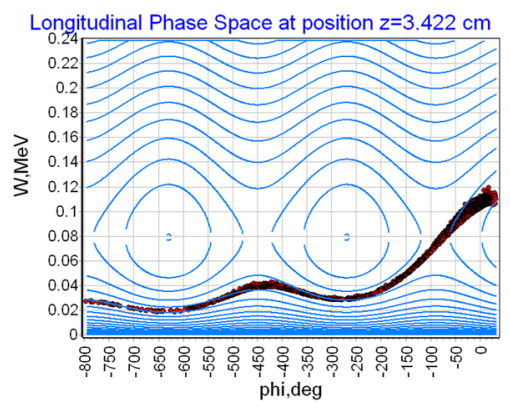

(f)

Longitudinal Phase Space at position $z=4.875 \mathrm{~cm}$

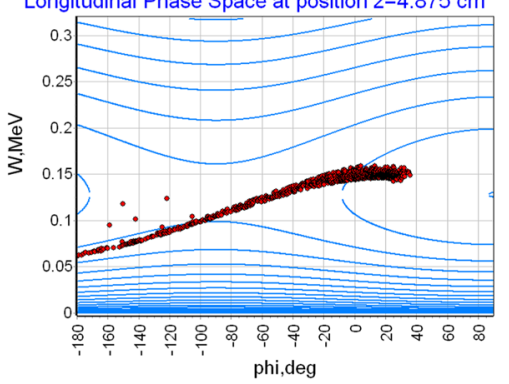

(h)

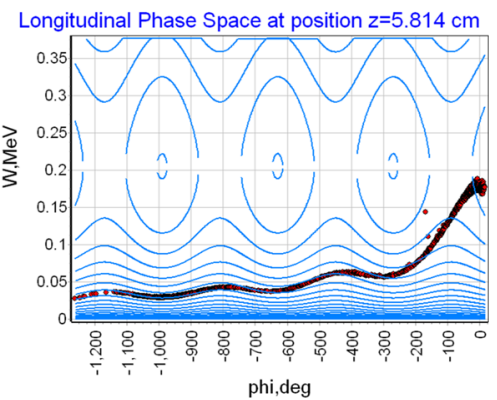

Fig. 32 Evolution of the beam phase space in the 180-keV Ku-band accelerator with a four-section $\beta_{\text {ph }}=$ const buncher, simulated in Hellweg with the consideration of transverse and space charge effects (thickening of beam lines). The groups of two plots demonstrate the beam at the entrance and at the end of each section: i.e., $\mathbf{a}, \mathbf{b}-\beta_{\mathrm{ph}}=0.3, \mathbf{c}, \mathbf{d}-\beta_{\mathrm{ph}}=0.5, \mathbf{e}, \mathbf{f}-\beta_{\mathrm{ph}}=0.6, \mathbf{g}, \mathbf{h}-\beta_{\mathrm{ph}}=0.7$ 
Table 5 Parameters of the $180 \mathrm{keV} \mathrm{Ku}$-band split linac for Co-57 replacement

\begin{tabular}{ll}
\hline Frequency & $16.4 \mathrm{GHz}$ \\
\hline Buncher type & Four sections with constant phase velocities \\
Phase velocity & $0.3-0.5-0.6-0.7$ \\
Field amplitudes (A) & $0.1-0.13-0.145-0.165$ \\
Input RF power & $50 \mathrm{~kW}$ \\
Unused RF power & $12.2 \mathrm{~kW}$ \\
Injection energy & $15 \mathrm{keV}$ \\
Length & $5.9 \mathrm{~cm}$ \\
Number of cells & 20 \\
Beam current & $\sim 5 \mathrm{~mA}$ \\
Beam energy (peak) & $179 \mathrm{keV}$ \\
Beam energy (mean) & $140 \mathrm{keV}$ \\
Beam transmission & $27.2 \%$ \\
\hline
\end{tabular}

energy, this part of the buncher must be made longer to improve the bunching effect. Numerical analysis of the beam dynamics, performed for the different configurations, demonstrated [24] that the bunching improves for lower field amplitudes since it leads to a larger number of phase oscillation and increases the length of the buncher. A similar result is observed for equilibrium velocities approaching $\pi / 2$.

It is interesting to observe the bunching around the equilibrium phase. For small amplitudes of phase oscillations, i.e., short initial bunches, the bunching coefficient linearly depends on the value of $\varphi_{\mathrm{s}}$, and the field amplitude does not affect the bunching efficiency. Particles located far from equilibrium have significantly lower bunching coefficient than particles located near $\varphi_{\mathrm{s}}$. Therefore, the bunching coefficient is lower for longer initial bunches, assuming the other parameters are the same.

In the most general case, both amplitude and equilibrium phase change along the bunching section. However, by assuming these parameters to be constant, it is possible to reach conclusions on their influence on bunching efficiency. Figure 33 shows that the bunching coefficient (phase compression) cannot exceed specific values for a given equilibrium phase, and when this phase approaches $\varphi_{\mathrm{s}}=0$, it can reach values of 18-20. In practice, this coefficient becomes significantly smaller since it is not reasonable to make $\varphi_{\mathrm{s}}=0$ because it results in very long bunching sections, and secondly, this value does not include the particles in the whole range of phases, $[-\pi / 2,3 \pi / 2]$, for a DC beam.

When the equilibrium phase is constant, the bunch should be shifted on the wave at the end of the buncher. As discussed in the previous section, this shift can be realized with a phase-shifting section with $\beta_{\text {ph }}<\beta_{\text {beam }}$. Good bunching performance can be obtained if the equilibrium phase is varied from 0 to $-\pi / 2$. The advantage of this technique is that it allows achieving $100 \%$ capture. To realize this method, it is necessary to choose the appropriate function of phase velocity change $\beta_{\mathrm{ph}}(\zeta)$. This function will also depend on the amplitude variation.

The simplest way is to use a linear function of $\varphi_{\mathrm{s}}(\zeta)$, while its derivative will be a function of field amplitude. Assuming that the most appropriate function for the equilibrium phase variation would be cosine, it is possible to write the expression for the phase velocity change:

$$
\varphi_{s}(\zeta)=\frac{\pi}{4}\left[1-\cos \left(\frac{\pi}{L} \lambda \zeta\right)\right]
$$


Fig. 33 Bunching (compression) coefficient as a function of equilibrium phase in the case of small amplitude phase oscillations

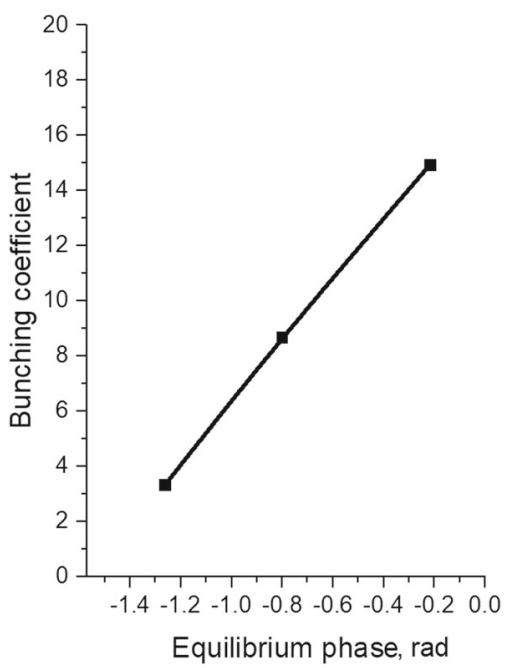

Here, $L$ is the bunching section length. It is necessary to perform analysis and monitor the electrons' trajectories numerically in phase space to control the equilibrium phase variation.

In order to get optimal beam parameters, one needs to numerically optimize the functions of $\mathrm{A}(\zeta), \beta_{\mathrm{ph}}(\zeta)$ and $\varphi_{\mathrm{s}}(\zeta)$, which is complicated even more by the fact that the length of the buncher is not constant and will depend on the energy gain. With enough time, skill and computational resources, a designer can do this either manually or by using some optimization algorithms [104]. At the same time, in the era before powerful computational tools, scientists developed a number of approaches, including using empirically obtained optimal functions of $\mathrm{A}(\zeta)$ and $\beta_{\mathrm{ph}}(\zeta)$ that can help rapidly develop tapered velocity bunchers. Although there are no universal solutions that will produce optimal beam parameters for any accelerator, these approaches might be useful as a starting point for buncher development. They can also be implemented into design optimization algorithms as they allow reducing the problem to 2-3 variables.

In the following sections, we will present three different approaches for tapered waveguide buncher design proposed by different Soviet scientists that include analytical expressions for buncher parameter (phase velocity and amplitude) variation and which can be useful for optimization algorithms and practical design.

\subsubsection{Direct formulas}

One of the simplest and most straightforward design approaches for tapered velocity bunchers was proposed by Prof. O.A. Valdner [23, 24]. A perfect buncher should provide the maximum capture of injected electrons, shortest phase length, and smallest energy spread while having a relatively simple and compact design. These requirements can be realized in waveguide bunchers with tapered velocity and amplitude of the EM wave. The bunching efficiency directly depends on the velocity and amplitude profiles $\beta_{\mathrm{ph}}(\zeta)$ and $\mathrm{A}(\zeta)$, which must be identified during the design.

Similar techniques to those used for $\beta_{\mathrm{ph}}=$ const bunchers can be applied for tapered waveguides, including amplitude and phase jump. To have efficient bunching, the initial phase velocity should be small and close to the velocity of the injected beam, and the equilibrium phase close to $\pi / 2$. For the amplitude profile optimization, it is necessary to account for 
the maximum values that depend on the available RF power source, which can be defined as $A_{\max }=\frac{\Lambda \sqrt{P_{\mathrm{RF}}}}{W_{0}}$, where $\Lambda=\frac{E \lambda}{\sqrt{P}}$ is an accelerating cell property that can be calculated for a particular geometry [19], $P_{\mathrm{RF}}$ is the RF power incoming into the cell, and $\mathrm{W}_{0}$ is the rest energy of the accelerated particle. Secondly, it is necessary to avoid sharp variations of $\beta_{\mathrm{ph}}(\zeta)$ and $\mathrm{A}(\zeta)$ because such jumps reduce the RF properties of corrugated waveguides and complicate their design, which in practice may lead to power reflections and breakdowns.

The general recommendations for the tapered velocity buncher design can be formulated in the following way. The dependence of $\beta_{\mathrm{ph}}(\zeta)$ should start with a section with $\mathrm{d} \beta_{\mathrm{ph}} / \mathrm{d} \zeta=$ 0 . Such a section will significantly improve the capture efficiency. Then, $\beta_{\mathrm{ph}}$ should increase with $\zeta$ to ensure phase oscillations for simultaneous bunching and acceleration. At the end of the buncher, the phase velocity is chosen to shift the bunch on the wave crest. This ensures efficient acceleration in the $\beta_{\mathrm{ph}}=1$ section.

Unfortunately, the optimal functions $\beta_{\mathrm{ph}}(\zeta)$ and $\mathrm{A}(\zeta)$ cannot be derived analytically. However, some experimentally obtained expressions [23] that satisfy the conditions mentioned above can be used for the practical design of a tapered buncher. The phase velocity profile can be calculated as:

$$
\beta_{\mathrm{ph}}(\zeta)=\frac{2}{\pi}\left(1-\beta_{\mathrm{inj}}\right) \operatorname{atan}\left(k_{1} \zeta^{k_{2}}\right)+\beta_{\mathrm{inj}}
$$

where

$$
\begin{aligned}
& k_{1}=3.8 \cdot 10^{-3}\left(10.8^{A_{M}}-1\right) \\
& k_{2}=1.25 A_{M}+2.25
\end{aligned}
$$

For further improvements in bunching efficiency, the amplitude should also vary along the buncher as:

$$
\begin{aligned}
& A(\zeta)=k_{3}-k_{4} \cos \left(\frac{\pi}{k_{5}} \zeta\right) \text { for } 0<\zeta<k_{5} \\
& A=A_{M} \text { for } \zeta \geq k_{5}
\end{aligned}
$$

Here

$$
\begin{gathered}
k_{3,4}=0.5 A_{M} \pm 0.15 \sqrt{A_{M}} \\
k_{5}=\frac{1}{1.25 \sqrt{A_{M}}}
\end{gathered}
$$

$A_{M}$ is the amplitude of the EM field in the accelerating section. These expressions can be used for amplitudes $A_{M}$ from 0.1 to 4.0 , initial phase velocities $\beta_{\mathrm{ph}}(\zeta)$ from 0.3 to 0.6 , and frequency bands from $\mathrm{L}-(1.3 \mathrm{GHz})$ to $\mathrm{Ku}-(16 \mathrm{GHz})$.

As an example, assume a 6-MeV C-band $(5.712 \mathrm{GHz})$ accelerator with a 5-MW power source and $50 \mathrm{kV}$ DC gun $\left(\beta_{\text {inj }}=0.412\right)$, and compare different buncher designs obtained using formulas (44)-(47) for different values of $A_{M}$. For the sake of experiment, let us assume that the linac has a focusing solenoid of $1000 \mathrm{Gs}$ and injection current of $100 \mathrm{~mA}$, so that the transverse RF and space charge repelling forces are kept under control, while the beam loading effect of the field is not dominant. Figure 34 compares phase velocity and amplitude profiles obtained by above-mentioned formulas, while Table 6 provides the results of beam dynamics simulations for these cases performed in Hellweg. These results suggest that there is an optimum value of $A_{M}$ that allows best transmission and energy spectrum. Lowfield (gentle) bunching obtained by these formulas does not seem to provide any significant benefits, while making the accelerating structure significantly longer. It is also interesting to 

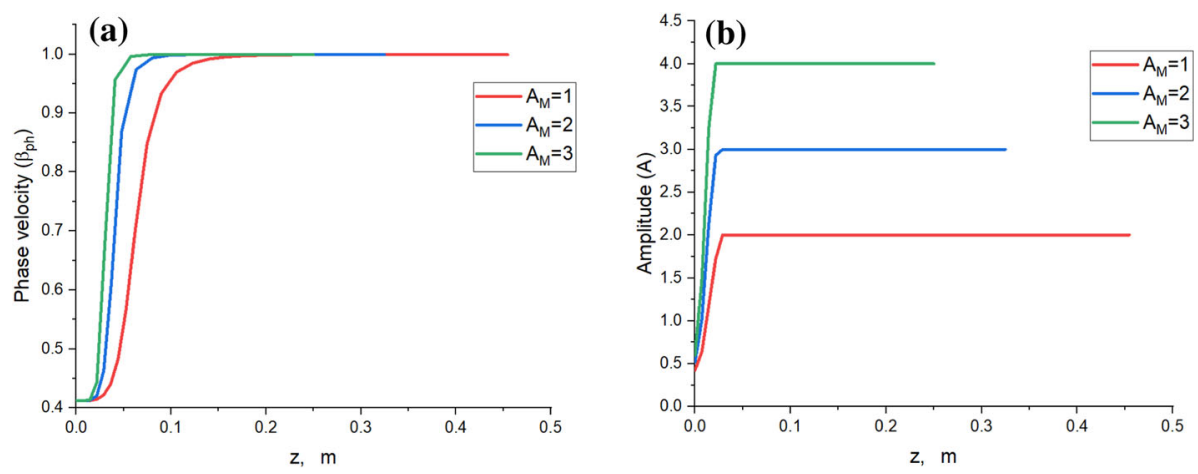

Fig. 34 Phase velocity a and amplitude b profiles obtained by formulas (44)-(47) for different values of $A_{M}$ in the case of a $6 \mathrm{MeV}$ (peak) C-band TW linac with 50-keV 100-mA DC beam injection

Table 6 Beam parameters of a 6-MeV (peak) C-band TW linac with 50-keV $100 \mathrm{~mA}$ DC beam injection and tapered velocity bunchers, designed according to Valdner's approach for different values of accelerating field $A_{M}$, as simulated in Hellweg

${ }^{\mathrm{a}}$ Here, we consider all cells with $\beta_{\mathrm{ph}}<0.999$ as a buncher section

\begin{tabular}{llll}
\hline Field amplitude $\mathrm{A}_{\mathrm{M}}$ & 2.0 & 3.0 & 4.0 \\
Field amplitude $\frac{E \lambda}{\sqrt{P}}, \Omega^{1 / 2}$ & 457 & 685 & 914 \\
Number of buncher cells $^{\mathrm{a}}$ & 18 & 10 & 7 \\
Number of total cells & 31 & 22 & 17 \\
Buncher length, cm & 22.7 & 9.8 & 7.5 \\
Linac length, cm & 45.4 & 32.46 & 24.9 \\
Mean energy after buncher, MeV & 2.20 & 1.07 & 0.57 \\
Peak energy after buncher, MeV & 2.70 & 2.4 & 0.74 \\
Transmission after buncher, \% & 40.58 & 45.6 & 55.0 \\
Energy spread (FWHM) after buncher, MeV & 0.49 & 0.27 & 0.24 \\
Mean energy after linac, MeV & 4.5 & 5.3 & 5.2 \\
Transmission after linac, \% & 40.2 & 42.7 & 41.4 \\
Energy spread (FWHM) after linac, MeV & 1.52 & 0.86 & 0.95 \\
Low-energy tail min energy, MeV & 3.4 & 4.4 & 3.9 \\
\hline
\end{tabular}

note that a high-amplitude buncher $\left(A_{\mathrm{M}}=4\right)$ provides very similar parameters to the optimal buncher $\left(A_{\mathrm{M}}=3\right)$, from which we can conclude that a trade-off between buncher complexity and beam parameters may be very much justified for industrial linac design if sufficient RF power is available.

Since Valdner's formulas have only one parameter to vary $\left(A_{\mathrm{M}}\right)$, they can provide only limited optimization and results, despite their simplicity. In fact, the $42.7 \%$ transmission obtained in Table 6 is not very impressive, although the results may differ much for different initial parameters (frequency, power, injection energy, etc.). In order to add more flexibility to these equations, one may substitute $\beta_{\text {inj }}$ with $\beta_{0}$ and vary it. The other option is to not use the chosen $A_{M}$ in the accelerating section but adjust it to the desired value, either smoothly or by an amplitude jump, to provide better acceleration efficiency. Finally, a designer can vary the coefficients in Eq. (44)-(47) (i.e., $3.8 \cdot 10^{-3}$, 10.8, etc.) to obtain better results. However, this might require the use of optimization algorithms. 


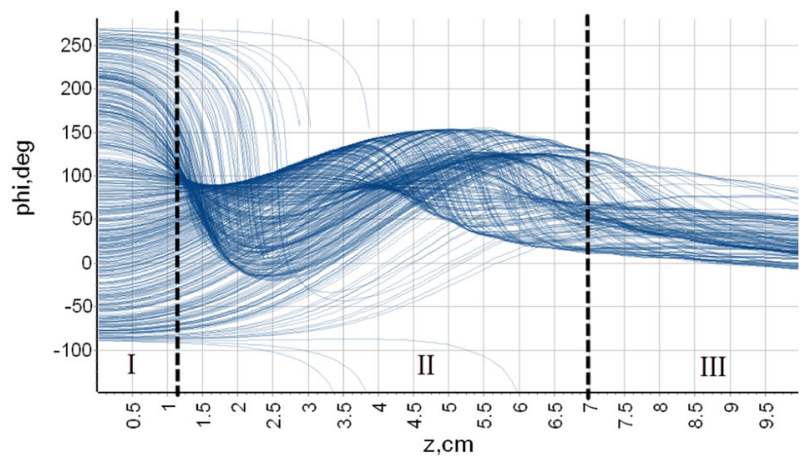

Fig. 35 Phase oscillations of the particles in a tapered wave buncher, simulated in Hellweg. The buncher can be separated into three sections: I-initial section for beam capture, II-middle section with intensive phase oscillations, and III-end section with minimal oscillations

\subsubsection{Buncher subsections}

Another more general and robust approach for a tapered buncher design was proposed by Dr. S.P. Lomnev [105]. This method requires numerical modeling that can provide phase portraits of the beam and implies the separation of the buncher into three subsections:

I. The initial section, where the particles are captured into the acceleration regime;

II. The middle section with intensive phase oscillations where bunches are formed;

III. The end section with minimal phase oscillations where the bunch parameters can be improved and the bunch is prepared for injection into the $\beta_{\mathrm{ph}}=1$ section for further acceleration.

In order to better understand this division, let us plot phase trajectories inside the buncher designed by the approach presented in the previous section using formulas (44)-(47) for $\beta_{\mathrm{ph}}(0)=0.412$ and $A_{\mathrm{M}}=1.6$. Figure 35 demonstrates phase oscillations in such a buncher, and all three subsections can be clearly identified: I-DC beam receives density modulation, II-intensive phase oscillations occur; III-phase oscillations decay and the bunch slips in phase along the wave. Now, we discuss the physics of each section and provide some practical guidance for their design.

The initial parts (I, II) of the buncher provide the most influence on the beam dynamics. Therefore, it is reasonable to understand the requirements for the bunch parameters entering the end section (III) to provide the best efficiency. So, we will start the description from the end of the buncher. Usually, this section can only utilize the variation in phase velocity (i.e., $A_{\text {III }}=$ const $)$. The function of this variation $\beta_{\mathrm{ph}}(\zeta)$ is defined by the energy of the entering bunch and the requirements for phase length and energy spread at the end of the buncher.

Assume $\beta_{\min }$ is the minimum velocity of the bunch particles entering section III and $\beta_{\max }$ is the maximum velocity. Then, it is possible to emphasize the following relationships for the phase and beam velocities:

1) $\beta_{\mathrm{ph}}=\beta_{\max }$. This case is useful when the fastest particle phase is minimal, and it is necessary to reduce the phase length of the beam. Alternatively, if the fastest particle has a maximum phase, it is possible to reduce the beam's energy spread in such a section. Also, the particles will reach the highest energy at the end of such a buncher.

2) $\beta_{\mathrm{ph}}=0.5 \cdot\left(\beta_{\min }+\beta_{\max }.\right)$. This design is the best for the simultaneous phase and energy flattening. 

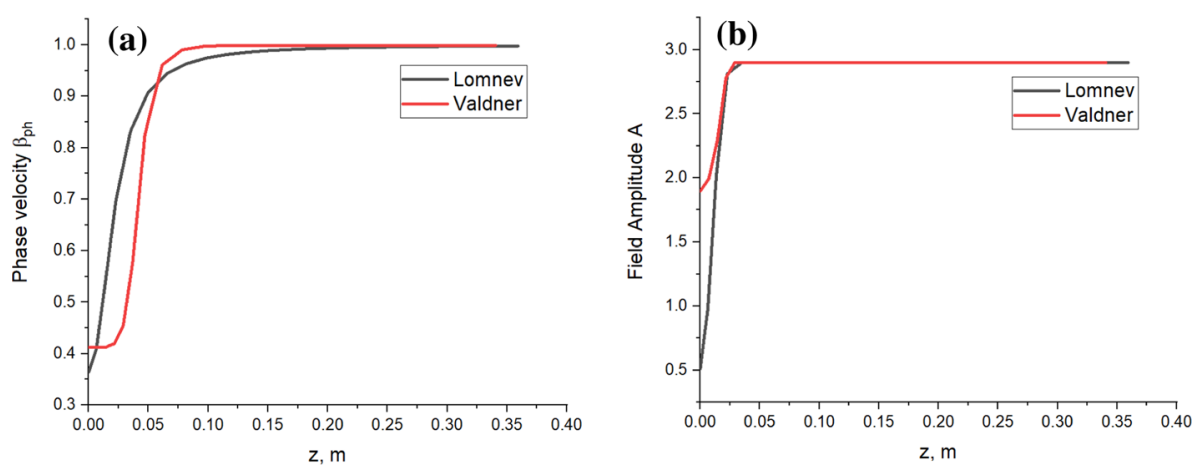

Fig. 36 Comparison of phase velocity $\mathbf{a}$ and amplitude $\mathbf{b}$ profiles obtained by Valdner's and Lomnev's formulas for $A_{M}=2.9$

3) $\beta_{\mathrm{ph}}=\beta_{\min }$. This design is reasonable to implement if the slowest particle has maximal phase, and it is required to compress the phase length of the bunch.

4) If the slowest particle has a minimum phase, it is reasonable to put it on the wave crest $(\varphi=0)$

It is interesting to note that it is possible to artificially induce phase oscillations for further bunch compression by implementing a phase velocity jump as described in Sect. 4.

For the middle section (II), it is reasonable to use another set of experimental dependencies [106]:

$$
\begin{gathered}
\beta_{\mathrm{ph}}(z)=\frac{2}{\pi} a \tan \left[n\left(\frac{z}{10}\right)^{2}+k_{1}\right] \\
A(\zeta)=A_{M} \sin \left[m\left(\frac{z}{10}\right)^{2}+k_{2}\right] \text { for } z<10 \sqrt{\frac{\left(\frac{\pi}{2}-k_{2}\right)}{m}} \text { and } A=\mathrm{A}_{\mathrm{M}} \text { otherwise }
\end{gathered}
$$

Here $m, n, k_{1}$, and $k_{2}$ are parameters that should be optimized to achieve the desired phase length and/or energy spread. "Appendix" D discusses the choice of these parameters. Units of length are in centimeters. The shape of the initial part of $\beta_{\mathrm{ph}}(\mathrm{z})$ and $\mathrm{A}(\mathrm{z})$ should be chosen to achieve maximum capture, while the end part is critical for bunching (phase length).

Now let us compare the results achieved with Lomnev's approach to Valdner's. For consistency, let us assume initial parameters similar to those discussed in the previous section: frequency $5712 \mathrm{MHz}$, RF power $5 \mathrm{MW}$, injection energy $50 \mathrm{kV}\left(\beta_{\mathrm{ph}}=0.412\right)$. This time we will consider that the accelerating section $\left(\beta_{\mathrm{ph}}=1\right)$ is made of a DLS with aperture radius $a=0.08 \lambda$ (typical numbers for DLS linacs [58]). By using the data from Fig. 14, we can estimate that the amplitude in this section is $A_{M} \sim 2.9,{ }^{3}$ which roughly corresponds to $\frac{E \lambda}{\sqrt{P}} \approx 650 \Omega^{1 / 2}$. In order to calculate phase velocity and amplitude profiles along the buncher, we will use Eq. (48),(49), assuming $k_{1}=0.6435, k_{2}=0.7126, \mathrm{n}=0.25, m=0.11$, as proposed by Prof. Lomnev in his book, but which are subject to further optimization for a particular design. These conditions yield the phase velocity and amplitude profiles shown in Fig. 36.

The results of beam dynamics simulations are presented in Table 7 and Fig. 37 along with the results achieved by Lomnev's and Valdner's formulas for the same initial parameters. By

3 This estimation does not include field amplitude attenuation due to RF losses or beam loading effects. However, both of these effects are accounted for in simulations. 
Table 7 Comparison of beam parameters achieved in a $6-\mathrm{MeV}$ C-band TW linac with 50-keV $100 \mathrm{~mA}$ DC beam injection and tapered velocity bunchers designed using Valdner's and Lomnev's formulas

\begin{tabular}{lll}
\hline Approach & Lomnev's & Valdner's \\
\hline Field amplitude $\mathrm{A}_{\mathrm{M}}$ & 2.9 & 2.9 \\
Number of unique cells & 22 & 11 \\
Total Number of cells & 22 & 23 \\
Initial phase velocity & 0.364 & 0.412 \\
Peak energy at the end of the linac, MeV & 6.0 & 6.0 \\
Mean energy at the end of the linac, MeV & 5.35 & 5.31 \\
Transmission after linac, \% & 51.3 & 43.1 \\
Energy spread (FWHM) after linac, MeV & 1.14 & 0.86 \\
Low-energy tail lowest energy, MeV & 3.8 & 4.3 \\
\hline
\end{tabular}

(a)

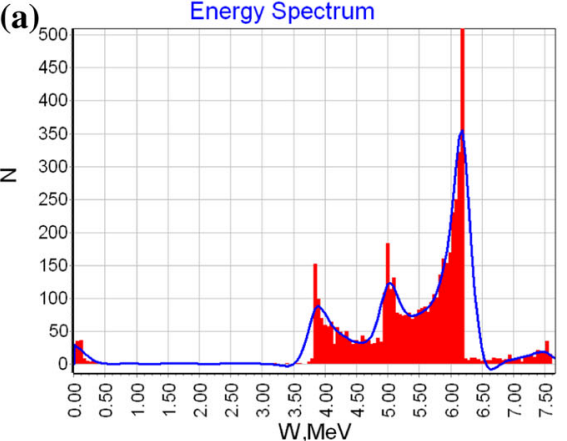

(b)

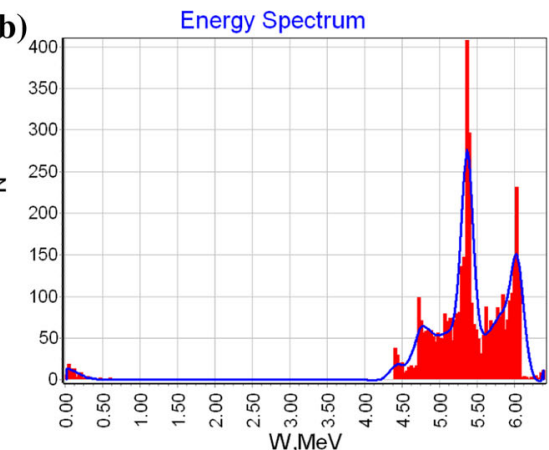

Fig. 37 Comparison of beam energy spectra at the exit of 6-MeV C-band linacs with tapered velocity waveguides designed using Lomnev's a and Valdner's b formulas

comparing these results, we can make the following conclusions: Lomnev's design is more complex as it requires more unique cells. However, a more smooth velocity profile leads to a better beam transmission (by $8 \%$ ), but $30 \%$ wider energy spectrum and longer lowenergy tails. At the same time, the most probable energy after Lomnev's buncher matches the maximum energy of the beam core, while in Valdner's design the most probable energy is shifted toward the mean energy. As discussed in previous sections, this effect depends on the injection phase into the accelerating section. Finally, Lomnev's design has a pronounced high-energy tail that might be very undesirable for some applications (as will be discussed in Sect. 4.1). The provided results may be improved by optimizing the parameters $k_{1}, k_{2}, \mathrm{n}$ and $\mathrm{m}$.

The provided analysis of the tapered velocity buncher designs shows that it is almost impossible to achieve good phase length and energy spread of the beam while capturing $100 \%$ of the particles. However, the practical designs of electron linacs do not imply such requirements. Usually, either a short phase length of the beam or a narrow energy spectrum is required. Moreover, most industrial applications do not require large beam transmission, and values in the range of $30-40 \%$ can be satisfactory as a trade-off for simplicity and low energy spread. 


\subsubsection{Machine learning}

An even more general approach based on the use of optimization algorithms was proposed by Prof. N.P. Sobenin in his Reference book on DLS accelerating structures [107]. Although this method is difficult or even unreasonable to use in a manual design approach, it can be implemented in machine learning algorithms, so it might be interesting for software developers. This approach allows using search engine optimization methods for complex multi-parametric objects [108]. Depending on the particular requirements for the bunch parameters, the optimization criteria can include beam transmission, energy spread, phase length, energy gain, tail lengths etc. As an illustration of this approach, let us consider the task for minimization of the energy spread at the end of the bunching section $\Delta \gamma_{\mathrm{b}}$.

The transition of continuous functions $\beta_{\mathrm{ph}}(\zeta)$ and $\mathrm{A}(\zeta)$ into a finite array is possible in different ways: for example, by formal power series intervals or piecewise-linear approximation. The main disadvantage of these methods is the large number of independent variables, which complicates the search and increases the time of the iterative procedure. Let us consider that the function of amplitude $\mathrm{A}(\zeta)$ is defined as a quadratic polynomial and the phase velocity has the form:

$$
\beta_{p h}(\zeta)=b_{1}-b_{2} e^{-b_{3} \zeta^{b_{4}}}
$$

where $b_{1-4}$ are independent variable for the iterative procedure. The disadvantage of this method is the limited class of applicable functions. However, this representation is convenient because it represents two types of functions: with and without points of inflexion. Moreover, it does not have a periodic behavior, which simplifies its practical realization.

Let us find the minimum of the beam's output energy spread as:

$$
F=\gamma_{i, \max }-\gamma_{i, \min }=f\left(b_{1}, b_{2}, b_{3}, b_{4}\right)
$$

Since the form of a function $\mathrm{f}$ is unknown, we have to represent it in the following polynomial form [109]:

$$
F=\alpha_{0}+\sum^{n} \alpha_{i} b_{i}+\sum_{i<j}^{n} \alpha_{i j} b_{i} b_{j}+\sum^{n} \alpha_{i j} b_{i}^{2}+\ldots
$$

with the regression coefficients $\alpha_{0}, \alpha_{\mathrm{i}}, \alpha_{\mathrm{ij}}, \alpha_{\mathrm{ii}}$, etc. By implementing the simulation results, it is possible to leave only sample regression coefficients $\alpha_{0}, \alpha_{\mathrm{i}}, \alpha_{\mathrm{ij}}, \alpha_{\mathrm{ii}}$, which will serve as approximations for theoretical regression coefficients. In order to define these coefficients, it is possible to use complete factorial experiment for four independent variables $b_{1-4}$ that are varied on two levels (planning of $2^{4}$ type). By approximating $\mathrm{f}$ as a linear function, it is possible to reduce the number of experiments, using fraction replications of complete factorial experiment ( $2^{4-1}$ planning). Then, for regression analysis, it is possible to write a simple formula [107]:

$$
\alpha_{i}=\sum_{n=1}^{b} \frac{y_{n} b_{\text {in }}}{8}, i=0-4
$$

All regression coefficients in the factorial experiment are evaluated independently. Since all variables are changed simultaneously during all experiments, each regression coefficient is defined by the results of all $N=8$ experiments, meaning that the dispersion in the coefficient estimation is 8 times lower than the dispersion of the experiment. During the iterative procedure, a series of experiments is performed for a local description of the result surface 
function as a linear polynomial. The motion along this surface is performed in the direction of its gradient, which can be described as:

$$
\operatorname{grad}(f)=\frac{\partial f}{\partial b_{1}} \vec{i}+\frac{\partial f}{\partial b_{2}} \vec{j}+\frac{\partial f}{\partial b_{3}} \vec{m}+\frac{\partial f}{\partial b_{4}} \vec{k}
$$

where $i, j, m, k$ are unity vectors in the direction of the coordinate axes. If the resulting surface can locally be represented as a linear equation, then partial derivatives are equal to the regression coefficients [107]: $a_{i}=\frac{\partial f}{\partial b_{i}}$. Further motion along this surface in the direction of a linearly approximated gradient must be accompanied by adjustment of the independent variables proportionally to the values of the regression coefficients, taking into account their sign. Therefore, the iterative procedure is chosen to maximize its convergence speed. In this particular task, the form of the result function is unknown, so in order to increase the speed of the search, it is recommended to take into account only the sign of the derivative, not its value, i.e., to use step normalization. In this case, the iterative sequence will have the form of:

$$
b^{m+1}=b^{m}-q \cdot \operatorname{sign}\left(\frac{\partial f}{\partial b_{i}}\right)
$$

The presented algorithm is very versatile and can be used for other design criteria such as beam transmission and bunch phase length. The further discussion of machine learning techniques is, however, beyond the scope of this paper [110, 111].

\section{Additional considerations for buncher design}

\subsection{Beam tails and energy spread}

In the previous chapter, we have already discussed the phenomena of energy tails and their nature. In this section, we would like to discuss this problem in more detail, including their effect on beam quality and some techniques for their elimination. Here, we also limit the discussion to industrial applications and will not consider scientific or FEL accelerators, where the requirements and effects of beam tails are generally different.

First, let us clarify that beam tails are the particles outside the beam core (for example, defined as FWHM), as shown in Fig. 38. There are two types of beam tails, which have different nature and effects on the accelerator performance: low-energy tails and high-energy tails. Low-energy tails usually appear when the particles found themselves outside of the RF bucket (especially high-phase-velocity buckets), when they can still be accelerated to a limited energy, before being decelerated (see, for example, lines (1) in Fig. 8). Depending on their initial phase distribution, they can form a continuous beam tail like in Fig. 26b or have a low-energy cut-off like in Fig. 38b. Since the trajectories of these particles are outside of the bucket, these particles will slip along the phase of EM and overlap with the successive bunches. At the same time, since their momentum is positive, these particles will not be lost and will eventually reach the end of accelerator.

In view of this nature, we can highlight several adverse effects caused by low-energy tails. First, due to their phase and velocity distribution, they can fill the whole accelerating structure and create an electron cloud that can affect the performance of high-current accelerators, for example, leading to an increase of the transverse and longitudinal emittance (beam size and beam divergence), reducing the accelerating field gradients due to space charge effects, and causing beam losses [112]. 
(a)

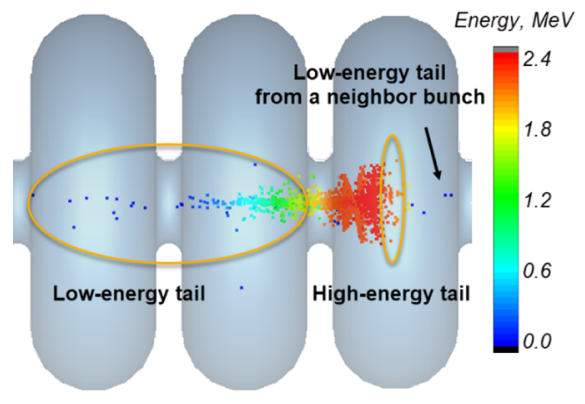

(b)

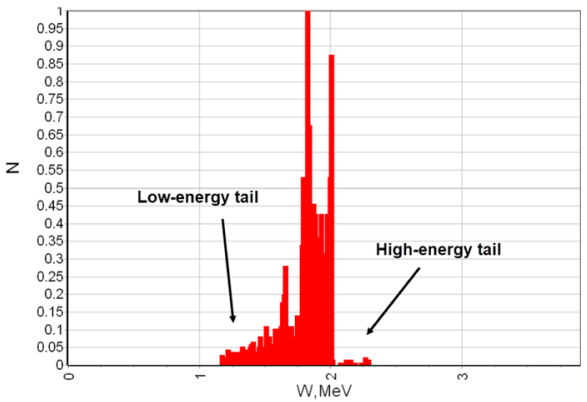

Fig. 38 a Illustration of low- and high-energy tails in longitudinal cross section of the beam inside ARCIS linac [95] as simulated in CST Particle Studio and b energy spectrum plot of this beam as simulated in Hellweg

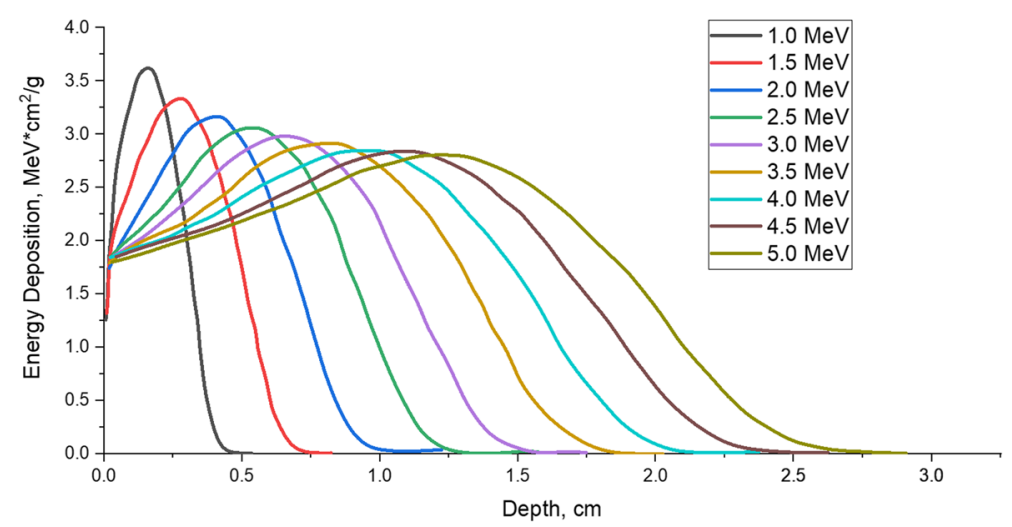

Fig. 39 Electron beam energy deposition in a layer of water for monochromatic electron beam with different energies

Secondly, these electrons still interact with the RF fields and receive the energy from them, causing beam loading effects (see Sect. 4.2) that reduce the accelerating voltage and lead to energy under-gain of the whole beam. This basically means that part of the RF power is used to accelerate particles that may not be used in the accelerator application. For example, in food, materials irradiation, or radiotherapy [113], low-energy electrons only irradiate the surface of the object. For example, Fig. 39 [21] demonstrates the energy deposition of an electron beam in a layer of water, showing that low-energy beams do not deposit their energy deep into thick objects. In the case where the electron beam is converted into X-rays, lowenergy electron tails will increase the low-energy tails of the bremsstrahlung [114], leading to a similar effect. Although these effects are harmless for most of the applications, low-energy tails generally reduce the efficiency of the accelerator and should be reduced or eliminated.

Unlike low-energy tails, high-energy particles appear because of large-amplitude phase oscillations, when some may find themselves at the top part of the RF bucket (see, for example, Fig. 30), and then accelerate faster than the beam core in a $\beta_{\mathrm{ph}}=1$ section (i.e., "deeper" trajectories (3) versus trajectory (2) in Fig. 8. The presence of high-energy particles in the beam can cause more severe effects for some application than the lost efficiency caused by low-energy tails. For example, high-energy electrons can produce neutrons via nuclear reactions [115, 116], which can lead to component activation [117]. For example, USDA 
limits the energy of electron beams to $10 \mathrm{MeV}$ and X-rays to $5 \mathrm{MeV}$, for food treatment [118]. The other problem is related to masking of detector signals in inspection systems by either neutrons or high-energy bremsstrahlung X-rays [96]. In sensitive detection techniques, such as enriched uranium detectors via beam-induced radiation [119], high-energy photons $(185 \mathrm{keV})$ can mask the passive decay of U-235.

Therefore, it is necessary to carefully control the beam quality during the accelerator design even for industrial-class machines and to take into account the requirements of the particular application and the accelerator operational environment. In the previous sections, we have already discussed some techniques to reduce beam tails, such as the over-bunching technique (see Sect. 3.3.2). In the following sections, we will discuss the other techniques that can be used in bunchers to reduce the beam's energy spread and beam tails.

\subsubsection{RF chopper}

The radical method to eliminate beam tails and reduce phase (and therefore energy) spread is to physically remove part of the beam before injection into the waveguide bunching or accelerating sections, i.e., right after DC gun or after the pre-buncher. Although this method can significantly reduce the injected beam current, it eventually increases the accelerator power efficiency since no power is wasted on accelerating low-energy tail particles.

In practice, the removal of the excessive particles can be achieved with the help of an RF chopper (an RF cavity followed by a slit). The mechanism of the RF chopper is somewhat similar to the pre-buncher, with the exception that the particles receive transverse velocity modulation, instead of longitudinal. Transverse modulation is then transferred into deflection after passing the drift space. If we place a mask screen with a hole in it, those particles whose deflection (which is a function of RF phase) is larger than the slit will be eliminated.

In order to explain the transverse modulation process, we write below the equation of the temporal transverse motion (along the $y$-axis, perpendicular to longitudinal z-axis,) of the particles exiting the deflector [120]:

$$
y(t)=k \frac{e V_{0}}{p_{0} c} \cos (\omega t)+k \frac{e V_{0}}{p_{0} c} \frac{2 \pi}{\lambda} \sin (\omega t) z
$$

where $V_{O}$ is the peak RF deflecting voltage, $p_{0}$ the particles momentum, $c$ is the speed of light, $k$ is a constant depending on beam and lattice parameters (such as beam emittance and beta function), and $z$ is the direction of propagation. This is an approximated formula with a first-order development of the sinusoidal transverse voltage. Notice that the further away the location of the mask $\left(z=z_{M}\right)$ is from the deflector, the wider the beam oscillation is. In Fig. 40, we show an example of such a motion. The transverse sinusoidal behavior of the beam exiting the deflector increases in amplitude until the mask is reached. By choosing the slit diameter and deflecting voltage, it is then possible to chop the $\mathrm{CW}$ beam and obtain aligned bunches of certain phase lengths at frequency $2 f_{0}$. In order to reverse the beam divergence, a focusing element can be placed after the RF chopper.

Figure 41 provides an example of beam phase volume evaluation of a 100-kV DC beam injected into a 10-MV/m S-band accelerating structure (similar to Sect. 3.1) with and without phase chopping. Although RF choppers can significantly improve the quality of the beam, they require a deflecting RF cavity operating at sub-harmonic frequency and additional beam optics, which can significantly complicate the linac design and is usually utilized only in high-current stationary accelerators. 


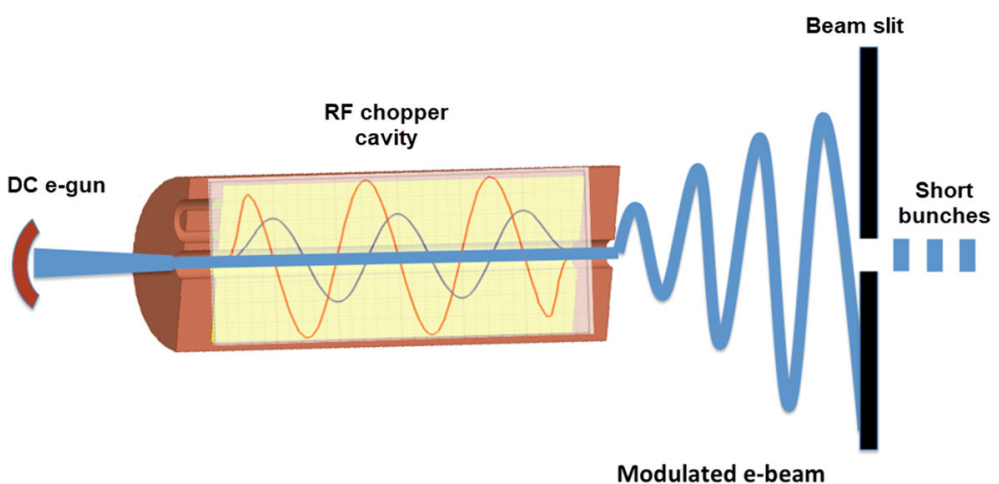

Fig. 40 Representation of RF chopper operation

(a)

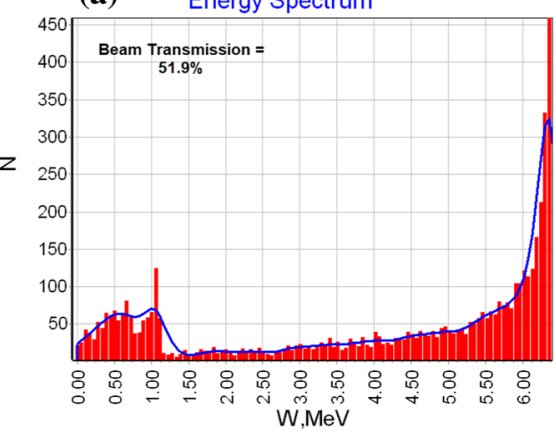

(c)

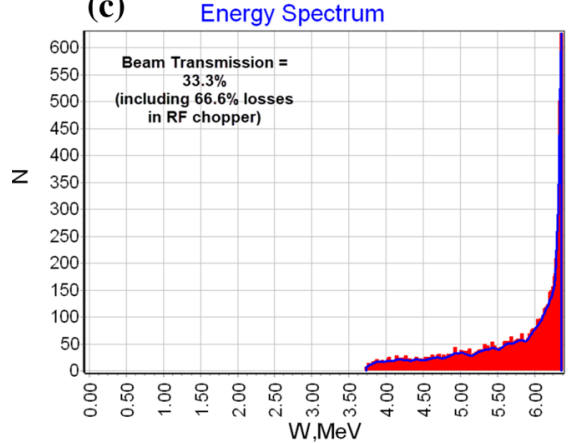

(b)

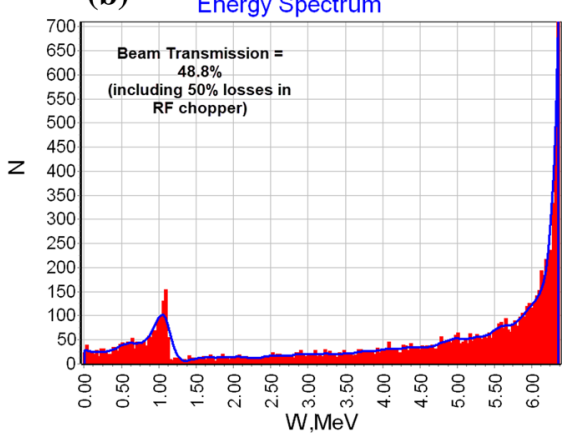

(d)

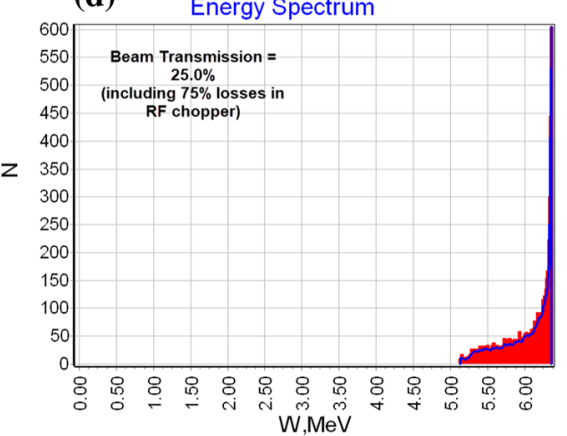

Fig. 41 Energy spectra plotted at the end of the linac for 100-keV DC beams injected directly into $10 \mathrm{MV} / \mathrm{m}$ S-band accelerating structure at $\pi / 2$ central phase as is (a), and with 180-deg (b), $120 \mathrm{deg}$ (c) and $90 \mathrm{deg}$ (d) chopping. Simulations performed in Hellweg

\subsubsection{Amplitude jump technique}

This technique significantly reduces the phase length of the bunch head, which will eventually transform to reduced energy spread during acceleration. This method requires the beam's injection to the accelerating waveguide section with a matched velocity and low amplitude. 
(a)

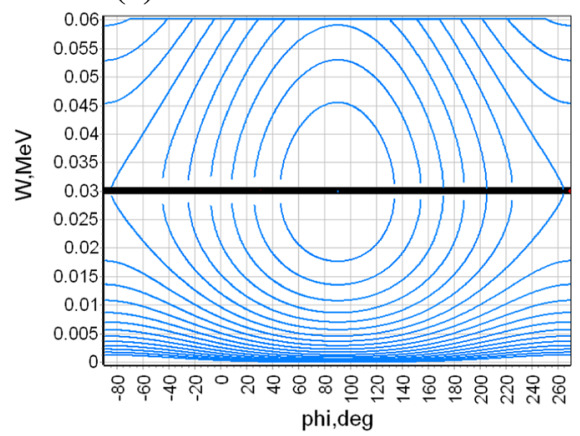

(c)

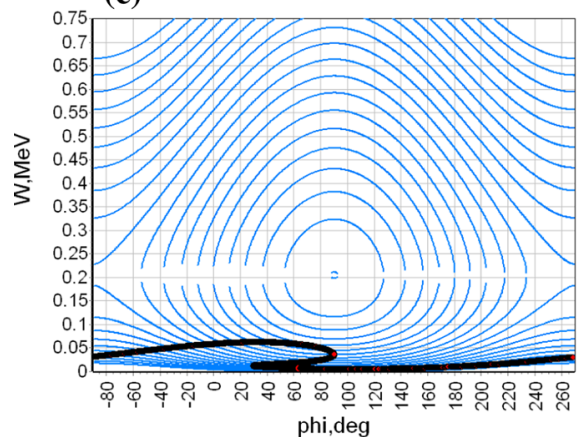

(b)
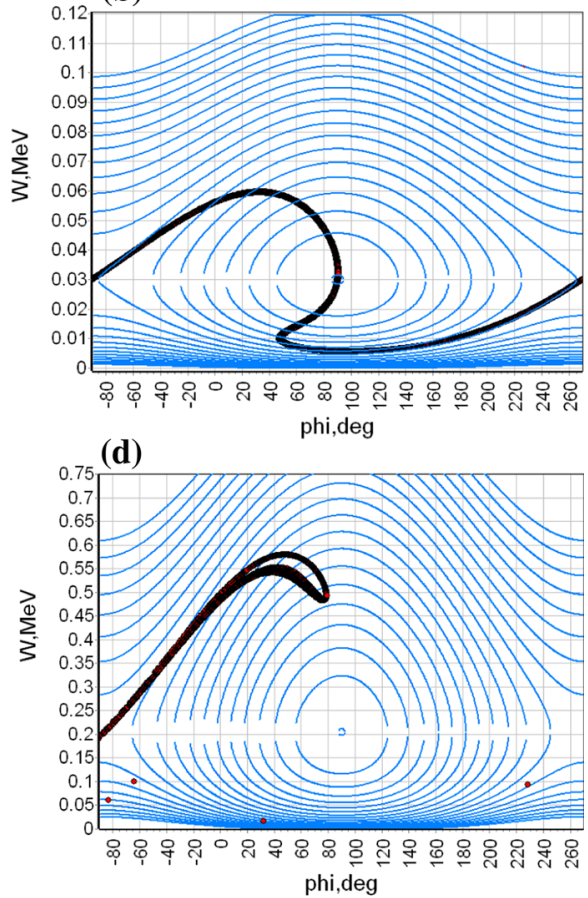

Fig. 42 Bunch compression with the amplitude jump technique. A DC beam is injected into an accelerating waveguide section with a matched phase velocity and low amplitude (a) and bunched into a minimum phase length (b). The amplitude is increased by a factor of 5 , and the phase velocity from 0.328 to 0.7 . The resulting bunch has smaller energy and phase spread than the one shown in Fig. 30

In this case, after passing a distance defined by the equation provided in "Appendix" C, the bunch's length will be minimized. At the same time, due to the low field amplitude, its energy spread will also be small. If at this point, we sharply increase the amplitude of the field by multiple times, the electrons inside the bunch head will find themselves inside the phase trajectory with much smaller phase oscillation amplitude. Further motion in the accelerating waveguide section will lead to the elimination of the beam tail. However, bunchers built with this technique have poor RF efficiency since long sections with low phase velocities are undesirable, as we discussed earlier.

A more practical implementation of this technique would be to sharply increase the phase velocity simultaneously with the amplitude, as shown in Fig. 42. Most of the particles will start accelerating on nearby phase trajectories, leading both to phase and energy spread compression, compared to DC injection into a $\beta_{\mathrm{ph}}>\beta_{\text {inj }}$ section. In this case, the first accelerating waveguide section will play the role of an integrated pre-buncher.

In general, the amplitude jump technique can be used multiple times during the bunching in both directions to achieve very clean beams (no tails and small head size). However, this method is technologically complex and can result in considerable beam losses after every jump. 


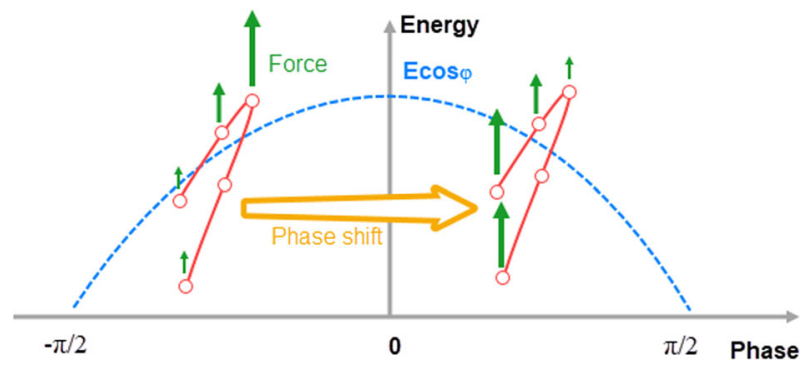

Fig. 43 Illustration of beam phase jump concept. In the initial bunch (left), head particles receive higher acceleration rate than tail particles, which leads to further bunch lengthening. By putting the bunch on the opposite side of the crest (right), the force distribution changes, and the energy spread reduces

\subsubsection{Phase jump technique}

To achieve good acceleration efficiency, the bunch should be accelerated on the crest of the wave ( $\varphi=0$, as shown in Fig. 8). This statement is particularly valid for bunches with short phase length and small energy spread. However, some bunchers yield bunches with large phase and/or energy spread. The best example of such a case is a $\beta_{\mathrm{ph}}=1$ buncher that produces beams with continuous and very long low-energy tails, as shown in Fig. $27 \mathrm{~d}$. The energy spread in such a beam will increase during acceleration since slower electrons occupy phases further from the crest and thus receive a smaller energy gain.

To compensate for the energy spread and stop the beam widening, it is possible to shift the bunch ahead of the crest $(\varphi>0)$, so that the slower particles are accelerated in higher field regions as shown in Fig. 43. By utilizing the field temporal distribution nonlinearity (phase-dependence as $E \cdot \cos \varphi$ ), it is possible to choose the phase shift value such that the slower particles shift to a higher field region than the faster particles, thus reducing the energy spread in the bunch. This method can be particularly efficient for long bunches.

It should be mentioned that the phase shift can be obtained not by a sharp jump but smoothly. To do this, a phase-shifting section with $\beta_{\mathrm{ph}}<\beta_{\text {beam }}$ should be inserted in the accelerating waveguide. A smooth phase shift also provides better energy compression compared to an acute phase change, and technologically it is easier to implement, since it does not require extensive changes in the accelerating waveguide parameters.

Figure 44 presents a comparison of measured 4-MeV beam's energy spectra for three cases [24]: with no phase jump (black), with three-phase jumps, followed by acceleration in the same-length accelerating waveguide (red), and an improved case with a smooth phase shift (blue). These results demonstrate that a smooth jump technique compresses the bunch better and improves its acceleration efficiency.

As an example of a combination of amplitude and phase jump techniques, it is interesting to present the concept proposed by Prof. B.S. Ishkhanov et al. [121]. In their design, they inject a $50-\mathrm{kV}(\beta=0.412)$ beam into a $\mathrm{SW}$ accelerating cell with a higher beta $\left(\beta_{\mathrm{ph}}=0.75\right)$ low-field $(A=0.38)$ cell, followed by a lower-beta $\left(\beta_{\mathrm{ph}}=0.55\right.$ and 0.8$)$ high-field cells $(A=2.36)$, before entering the regular accelerating section. The phase jump is accomplished by the insertion of a low-beta (0.55) cell in between the high-beta cells ( 0.75 and 0.8$)$. This design resulted in $64.5 \%$ beam transmission and $0.35 \mathrm{MeV}$ FWHM energy spread at the end of $10 \mathrm{MeV}$ linac.

A similar technique was used in a C-band 5-10 MeV linac, developed by ScanTech Sciences LLC for a cargo inspection system [123], shown in Fig. 45. Here, a low-beta 


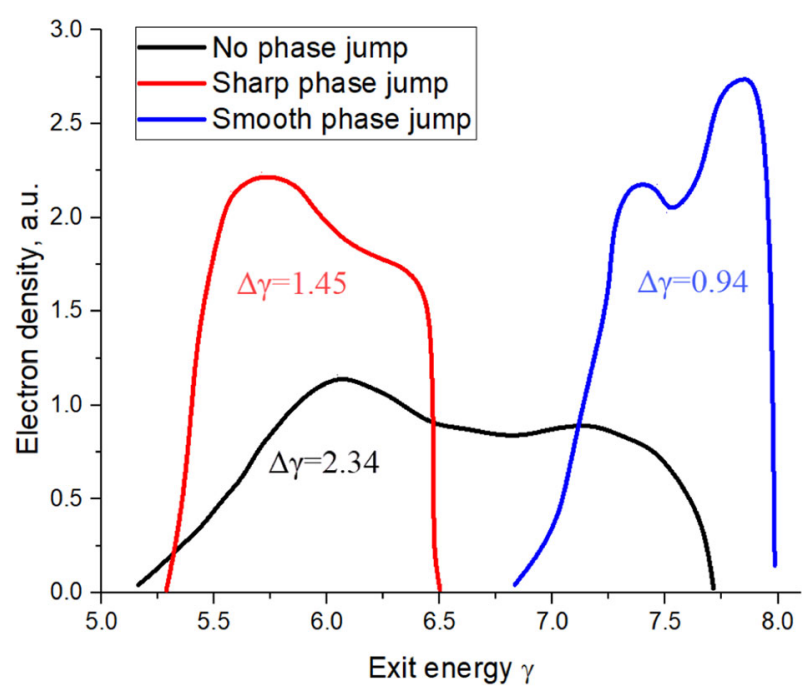

Fig. 44 Energy spectra of bunches accelerated in a $\beta_{\mathrm{ph}}=1$ section with no phase jump technique (black) and with sharp (red) and smooth (blue) jumps. The smooth jump not only compresses the bunch better but also improves its acceleration efficiency

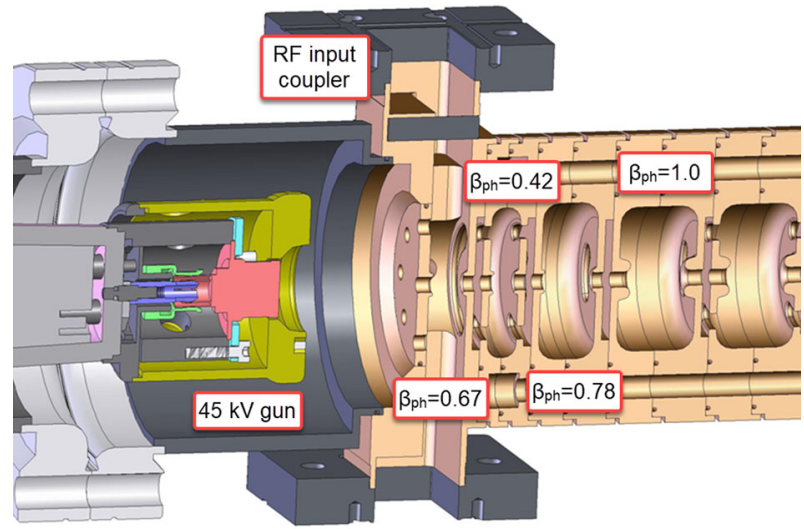

Fig. $453 \mathrm{D}$ model of the injector and bunching section of a SW C-band 5-10 MeV electron linac, using amplitude and phase jump techniques, developed by ScanTech Sciences, LLC [122]

$\left(\beta_{\mathrm{ph}}=0.42\right)$ cell is located between $\beta_{\mathrm{ph}}=0.67$ and $\beta_{\mathrm{ph}}=0.78$ cells to provide a phase jump. The amplitude ratio between the first and second cells of 0.12 provides and amplitude jump with an energy compression. This design provides $57.1 \%$ beam transmission with $\sim 0.36 \mathrm{MeV}$ FWHM energy spread.

\subsection{Beam interactions with accelerating structure}

Since the bunchers are rarely self-consistent devices (except the cases where the whole structure represents a buncher, such as ultra-low-gradient or sub-MeV linacs), their performance is closely related to further acceleration. This section will briefly discuss how the beam parameters after the buncher can influence the accelerated beam parameters. 
The electrons become relativistic after the buncher and then accelerate in the sections with $\beta_{\mathrm{ph}}=1$. Due to the small variation in the beam and phase velocities, the phase oscillations stop, and the electrons slowly slide along the wave. For multi-MeV accelerators, the accelerating section length significantly exceeds the buncher length. Therefore, it is fair to say that the $\beta_{\text {ph }}=1$ section is exclusively used for acceleration. To properly calculate beam dynamics in this section, it is necessary to consider the following essential factors. The accelerating field amplitude in TW structures attenuates via two mechanisms: (1) due to the power losses in the accelerating waveguide walls and (2) because the wave transfers its energy to the beam:

$$
\frac{\mathrm{d} P_{E M}}{\mathrm{~d} z}=-2 \alpha \cdot z-I \cdot E_{z}
$$

Here $\alpha$ is the field attenuation decrement (see Fig. 14) and I is the current of the accelerated beam.

These factors lead to the fact that the analytical equations of the electron motion become much more complex and not very illustrative. For example, Eqs. (9) and (10) will now have the form:

$$
\begin{gathered}
\frac{d \beta}{d \zeta}=\frac{\left(1-\beta^{2}\right) A \cos \varphi}{\gamma \beta} \\
\frac{d A}{d \zeta}=-\alpha(A+\mathrm{IR} \cos \varphi) \\
\frac{d \varphi}{d \zeta}=2 \pi\left(\frac{1}{\beta_{\mathrm{ph}}}-\frac{1}{\beta}\right)+\Omega I \sin \varphi
\end{gathered}
$$

Here $R$ and $\Omega$ are the parameters that characterize the beam interaction with the EM wave. More details, including their definition and calculation, can be found in [55, 87]. In order to solve these equations, the designer must use numerical analysis tools. The attenuation effects in TW structures can be compensated by a gradual increase of the "unloaded" amplitude, realized via shrinking the beam apertures (so-called constant gradient structures). Although this approach improves beam dynamics, it complicates the accelerating structure design. More details of the DLS structure design, including a comparison of constant impedance and constant gradient structures, can be found in [15].

As was discussed in Introduction section, in the SW structure, the amplitude decay has a different nature: the field does not decay as $e^{-\alpha \mathrm{z}}$ but is uniformly reduced in all accelerating cells due to both RF losses and beam loading (see Fig. 15). This difference should be taken into account for the proper linac design. More details on the difference between SW and TW structures can be found in [10].

\subsubsection{Beam loading effects}

Acceleration of a high-current beam reduces the power flow in the accelerating waveguide, the accelerating field amplitude, and by extension the output beam energy. For industrial accelerators, the beam power constitutes a considerable fraction of the input RF power. Also, high currents require proper consideration of the space charge forces acting on the bunch, since they are comparable to the forces of the RF fields at low energy. Space charge may influence the stability of longitudinal (phase) or transverse (radial) dynamics. Next, the motion of grouped bunches along the waveguide can induce a reactive current component on the waveguide walls, changing the phase velocity of the electromagnetic wave, which in turn degrades the beam quality and acceleration efficiency. Finally, the particles traveling off-axis can excite asymmetric waves with a transverse on-axis component. These waves deflect the 
beam away from the waveguide axis, which can lead to the beam-breakup (BBU) effect. This tutorial will focus only on the longitudinal beam-induced effects.

When bunches of electrons pass through a disk-loaded waveguide, not connected to any power source, they excite an electromagnetic wave inside this DLS with a phase velocity equal to the velocity of the bunch [124]. This effect is called a "wake field." In this excited wave, the maximum of the decelerating field corresponds to the center of the bunch. A similar effect is observed when the bunch passes through a SW cavity [125], with the exception that in a TW cavity the bunch can excite any mode within the DLS bandwidth due to the continuous dispersion curve, while in SW structures only discrete resonances are possible (see Sect. 2.5).

When the bunches pass through a powered accelerating structure, they still excite the wake field, which now interacts with the accelerating wave. Since the bunch is accelerated in the phase where $E_{\mathrm{RF}} \cdot \cos \varphi>0$, and its wake field is decelerating with a minimum always following the bunch center, the effect of this field superposition will result in a decrease in the accelerating field. As demonstrated in ref. [23], in stationary regime, the resulting beam energy in a DLS with a uniform geometry (so-called constant impedance) can be calculated as:

$$
W=\sqrt{\frac{2 r_{\mathrm{sh}} P_{\mathrm{RF}}}{\alpha}}\left(1-e^{-\alpha L}\right)-I_{b} r_{\mathrm{sh}} L\left(1-\frac{1-e^{-\alpha L}}{\alpha L}\right)
$$

Here $r_{\mathrm{sh}}$ is the shunt impedance of the DLS, $\alpha$ is the attenuation factor, $L$ is the length of the structure, $P_{\mathrm{RF}}$ is the input RF power and $I_{\mathrm{b}}$ is the average (per RF pulse) beam current. When the current is high enough, both terms become equal and negate each other. This corresponds to the theoretical maximum current that can be accelerated in a given structure:

$$
I_{\max }=\sqrt{\frac{2 \alpha P_{\mathrm{RF}}}{r_{\mathrm{sh}}}}\left(1-e^{-\alpha L}\right)
$$

It is also helpful to provide expressions for the energy in a beam-loaded constant gradient traveling wave structure [58]:

$$
W=\sqrt{r_{\mathrm{sh}} P_{\mathrm{RF}} L\left(1-e^{-2 \alpha L}\right)}-I_{b} r_{\mathrm{sh}} L\left(\frac{1}{2}-\frac{\alpha L \cdot e^{-2 \alpha L}}{1-e^{-2 \alpha L}}\right)
$$

and in a standing wave structure:

$$
W=\frac{\sqrt{r_{s h} P_{R F} L}}{\frac{I_{b}}{2} \sqrt{\frac{r_{s h} L}{P_{R F}}}+\sqrt{1+\frac{I_{b}^{2}}{4} \frac{r_{s h} L}{P_{R F}}}}
$$

The latter is provided for the optimal coupling strength between the accelerating structure and the RF input coupler (see [10] for more details).

If the bunches are not located on the crest of the accelerating wave, the beam loading effect will also cause the phase slippage of the beam, which is described in "Appendix" E. The effect of beam-induced phase slippage implies that the accelerating section has a maximum length, limited to the case when the beam slips into a decelerating phase. It is important to note that this effect occurs if the bunch is located off the crest of accelerating wave. A similar effect will happen if the frequency of the RF generator changes during the operation. This leads to an effective change of the phase velocity and leads to phase slippage if the particles move with a speed close to the speed of light. A similar effect also occurs when beam energy is varied by the phase jump technique. 


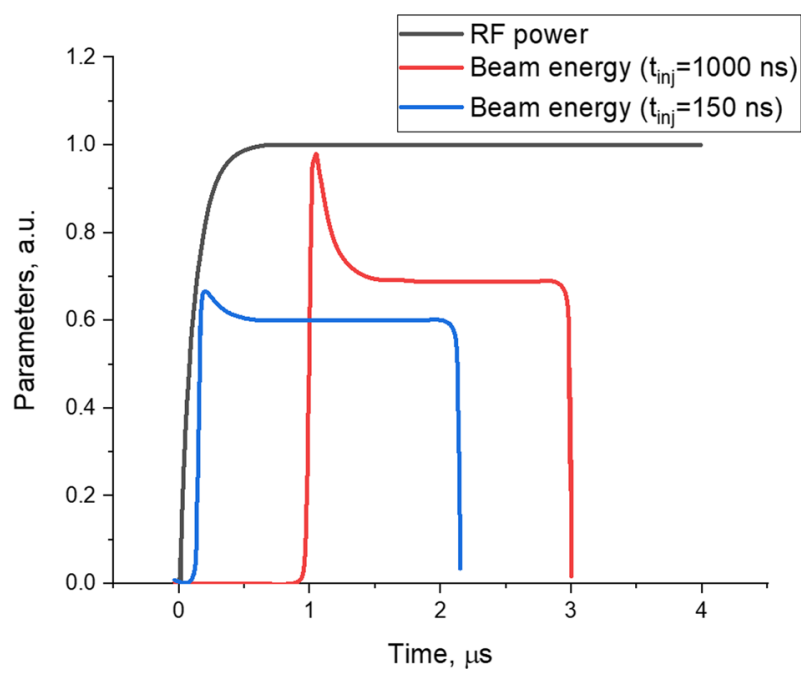

Fig. 46 Temporal profiles of the RF power (black) and beam energy in a TW accelerator with a filling time of $\sim 600 \mathrm{~ns}$. The beam is injected during the steady-state regime at $t=1 \mu \mathrm{s}$ (red) or during RF transient at $t=$ 150 ns (blue)

\subsubsection{Transient processes and acceleration of short bunches}

Since beam loading effects are caused by the interaction between the beam and the accelerating structure, they do not happen simultaneously but are defined by transient processes inside the structure. Therefore, it is important to understand the time frame of these processes.

In TW structures the transient time (or "filling time," i.e., the time required to fill the structure with the RF power) depends only on the structure length $L$ and group velocity as:

$$
\tau_{\mathrm{TW}}=\frac{L}{\beta_{\mathrm{gr}} c}
$$

When the structure is not homogenous, i.e., the group velocity changes from cell to cell as in constant gradient structures, this formula take the differential form $d t=\frac{d z}{\beta_{\mathrm{gr}}(z) c}$, and will require integration. For example, if the group velocity changes linearly from the input cell $\beta_{g r, i n}$ to the output cell $\beta_{\mathrm{gr}, o u t}$, Eq. (65) can be rewritten as:

$$
\tau_{\mathrm{TW}}=\frac{L \cdot \ln \left(\frac{\beta_{\mathrm{gr}, \text { out }}}{\beta_{\mathrm{gr}, \text { in }}}\right)}{c \cdot\left(\beta_{\mathrm{gr}, \text { out }}-\beta_{\mathrm{gr}, \text { in }}\right)}
$$

The filling time is both the time required for the accelerating fields to build up inside the cavity after turning on the RF generator, and for the beam loading effects to be established when the beam is turned on. For example, Fig. 46 demonstrates the beam energy profile in a generic accelerator with a filling time of $\sim 600 \mathrm{~ns}$. The RF power is applied at $t=0$ and starts to fill the structure, with a steady state achieved by the end of the filling. In one case (red), the beam is injected at $t=1 \mu \mathrm{s}$ (at steady state) and it requires another $600 \mathrm{~ns}$ for the beam loading effect to take place, resulting in high-energy particles in the accelerated beam. In the other case (blue), the beam is injected during the filling time of the structure, which allows to smoothen the overshoot effect and eliminate contamination of high-energy particles. 
At the same time, this effect can be used to accelerate short bunches of high beam current without the need to provide the amount of RF power that would otherwise be needed in the steady-state regime (i.e., $I_{\mathrm{b}} \cdot W$ ). As shown in Fig. 46, if the beam duration is shorter than the fill time of the structure, the beam loading will not have the full effect and the beam will get maximum energy even at high currents [126]. The formula to calculate the maximum current that can be accelerated in the short pulse regime is provided by [127]:

$$
I_{\max }=\frac{P_{\mathrm{RF}}}{W_{\max }} \cdot \frac{\tau_{\text {fill }}}{\tau_{\text {bunch }}}
$$

Although similar effects are observed in SW structures, their mechanism of filling with RF power is different. In particular, the filling time depends on the loaded Q-factor of the cavity. The loaded Q-factor takes into account the coupling of the external waveguide to the cavity, and the balance of RF power that flows into the cavity, RF power that reflects from the cavity, RF power that dissipates in the cavity walls and RF power that is radiated from the cavity back into the coupler [128]:

$$
\frac{1}{Q_{L}}=\frac{1}{Q_{0}}+\frac{1}{Q_{\mathrm{ext}}}
$$

Here $\mathrm{Q}_{0}$ is the intrinsic quality factor of the cavity, described in Sect. 1, which accounts for $\mathrm{RF}$ losses in the walls, and $\mathrm{Q}_{\mathrm{ext}}$ is the external quality factor that takes into account radiation from the cavity into the external circuit. The ratio of intrinsic and external Q-factors is called the coupling strength $\chi$ :

$$
\chi=\frac{Q_{0}}{Q_{\text {ext }}}
$$

When a beam is not present, this balance is achieved when $Q_{\text {ext }}=Q_{0}(\chi=1$, so called, critical coupling). However, when the beam is present, it takes a part of stored energy from the cavity and changes the balance, so that the radiated power becomes smaller. To restore this balance, the cavity must be overcoupled by the following value [58]:

$$
\chi_{\mathrm{opt}}=\left(\frac{I_{B}}{2} \sqrt{\frac{r_{\mathrm{sh}} L}{P_{\mathrm{RF}}}}+\sqrt{1+\frac{I_{B}^{2}}{4} \frac{r_{\mathrm{sh}} L}{P_{\mathrm{RF}}}}\right)^{2}
$$

At the same time, the RF power delivered to the cavity will depend on time as [10]:

$$
P_{\text {cavity }}(t)=P_{R F}\left\{1-\left[\left(1-e^{-\frac{t}{\tau}}\right) \frac{2 \chi}{1+\chi}-1\right]^{2}\right\}
$$

where the time decrement $\tau$ can be found as [27]:

$$
\tau=\frac{Q_{L}}{\pi \cdot f_{\mathrm{RF}}}=\frac{Q_{0}}{\pi(1+\chi) f_{\mathrm{RF}}}
$$

The actual filling time of an SW structure depends on the desirable level of RF fields that needs to be achieved and is usually $\tau_{\mathrm{SW}}=(2-3) \cdot \tau$. This expression sometimes leads to a false conclusion that by artificially increasing the coupling strength $\chi$, it is possible to reduce the filling time of the cavity and therefore make it more efficient. These considerations not only do not take into account the reflected power that will be wasted due to the cavity coupler mismatch, but also that over-coupled cavity (without proper beam loading, according to Eq. (70)) will have a temporal field profile as shown in Fig. 47, which means that although the operational values of the RF fields are achieved sooner, they continue to rise, leading to a 


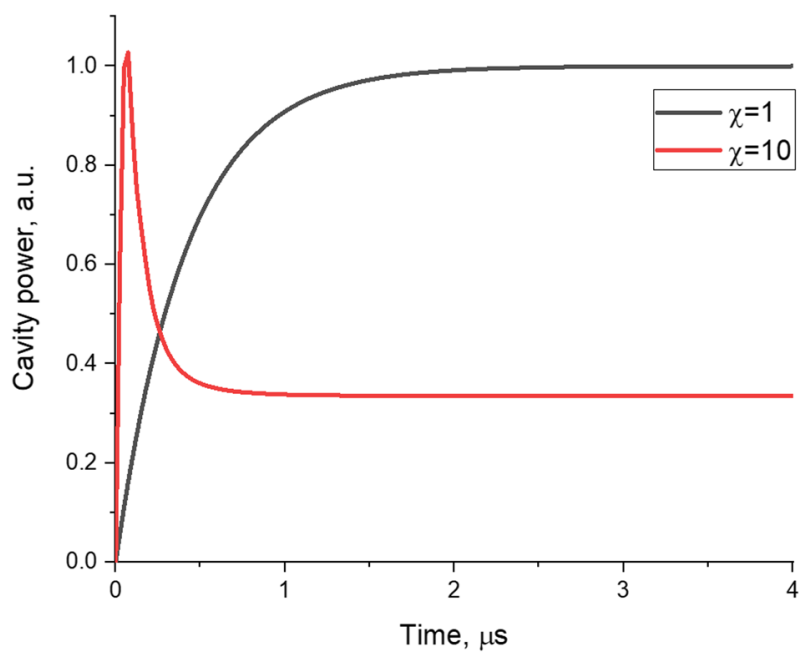

Fig. 47 Comparison of cavity power dependence in critically coupled (black) and overcoupled (red) S-band SW cavities with $Q_{0}=15,000$ after turning on RF power. It is clearly seen that the power overshoot can be significant and delay the expected steady-state regime. Note that in this example $>60 \%$ of the RF power is reflected for the case $\chi=10$

power overshoot as well as beam energy overshoot if injected simultaneously with RF power. However, if the cavity is optimally coupled, the beam loading will smoothen this overshoot.

\subsubsection{Phase slippage and optimal injection phase}

Despite the problem's complexity, we can provide some important results from numerical analysis that can help with the accelerating section's design. Let us assume that the buncher accelerates the beam to the velocity $\beta_{0}$ that differs from the speed of light by several percent and has a small phase length. Then, in long accelerating sections, the bunch phase will be sliding toward the asymptotic phase $\varphi_{\mathrm{a}}$ that can be found with [23]:

$$
\sin \varphi_{a}=\sin \varphi_{0}-\frac{2 \pi}{A} \sqrt{\frac{1-\beta_{0}}{1+\beta_{0}}}
$$

In this case, it is possible to consider that the energy gain in the acceleration section will be:

$$
\Delta \gamma \approx A_{M} \frac{L}{\lambda} \cdot \cos \varphi_{a}
$$

Obviously, $\gamma_{\max }=\mathrm{A}_{\mathrm{M}} \cdot L / \lambda$ is the maximal energy that electrons can gain is the accelerating section and $\Delta \gamma$ takes into account the bunch phase slip from the wave crest. Now, considering the expression for the asymptotic phase (16) that was obtained earlier, it is possible to calculate the energy under-gain due to the phase mismatch of the bunched beam:

$$
\frac{\Delta \gamma}{\gamma_{\max }}=\sqrt{1-\left(\sin \varphi_{0}-\frac{2 \pi}{A} \sqrt{\frac{1-\beta_{0}}{1+\beta_{0}}}\right)^{2}}
$$

Assuming that $\beta_{0}$ is defined and corresponds to $\gamma=6$, we can plot the energy under-gain as a function of the bunch phase for different accelerating field amplitudes (Fig. 48). For large 


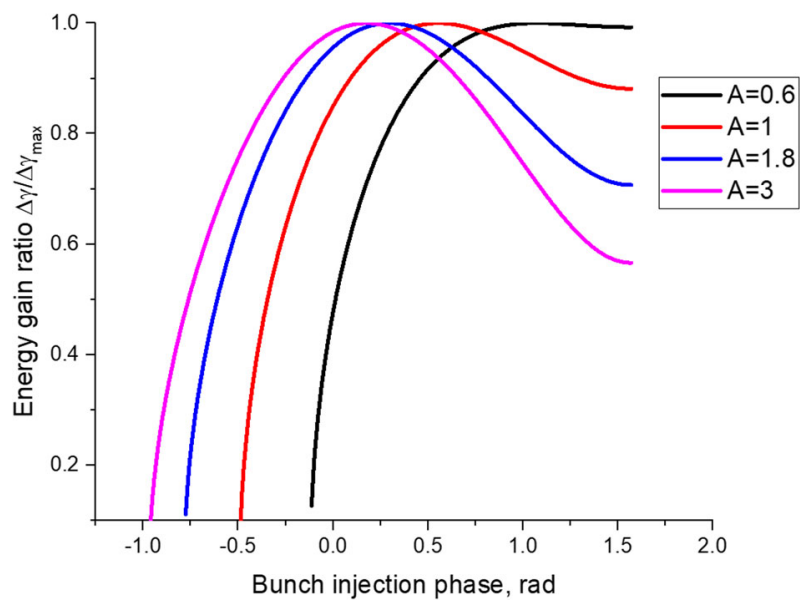

Fig. 48 Energy gain related to the maximum possible gain as a function of the $\gamma=6$ bunch injection phase for different amplitudes of the EM wave. This plot demonstrated the importance of a proper injection phase selection for efficient acceleration

amplitudes, the most efficient acceleration occurs at the wave crest. A somewhat counterintuitive result is obtained for low field amplitudes, where the most efficient acceleration will be achieved if the bunch is injected into the wave node $\left(\varphi_{0}=\pi / 2\right)$. For moderate amplitudes, the optimal phases are located ahead of the crest ( $\pi / 6$ for $A=1.0, \pi / 9$ for $A=1.8$, and $\pi / 18$ for $A=3.0$ ). Another conclusion is that the bunches with shorter phase lengths will have smaller energy spread at the accelerator's exit. Figure 48 can be used to calculate the phase to energy spread transformation in $\beta_{\mathrm{ph}}=1$ sections.

\subsubsection{Energy under-gain due to RF frequency mismatch}

In the conclusion of this tutorial, we will consider another effect that is common in industrial accelerators. In the previous sections, we considered that the frequency of the electromagnetic wave is constant and stable. However, in practice, the RF generator can have a frequency jitter or change frequency during operation due to temperature variations. Also, the frequency of the accelerating structure itself can change due to thermal effects [129]. The frequency change leads to a change in the EM wave's phase velocity since the disk-loaded waveguide has a dispersion [15]. The phase velocity change will lead to a shift of the electron bunch relative to the EM wave and, therefore, to energy under-gain.

Let us estimate the value of the energy under-gain, assuming that $\mathrm{A}(\mathrm{z})=$ const. For ultrarelativistic electrons, the phase slippage of the particles is negligible. Therefore, it is possible to assume that the electrons' velocity equals the phase velocity of the EM wave. In this case, the asymptotic phase equals the injection phase, which is reasonable to assume as $\varphi_{0}=0$. A frequency mismatch can change the phase velocity to be either higher or lower than the speed of light, but still constant along the accelerating waveguide. Since the particle will move with $\beta \approx 1$, its phase will change linearly with the travelled distance as $\varphi=\varphi_{0}+k_{1} \cdot \mathrm{z}$, where:

$$
k_{1}=\frac{d \varphi}{d z}=k\left(\frac{1}{\beta_{\mathrm{ph}}}-\frac{1}{\beta}\right) \approx k\left(\frac{1}{\beta_{\mathrm{ph}}}-1\right)
$$


For $\varphi_{0}=0$, the energy gain can be calculated as

$$
\Delta \gamma=\frac{A \cdot \sin k_{1} L}{k_{1}}=A \cdot L \frac{\sin \Delta \varphi}{\Delta \varphi}
$$

The phase slippage $\Delta \varphi$ can be estimated by calculating the dispersion of the accelerating waveguide $\mathrm{d} \beta_{\mathrm{w}} / \mathrm{df}$. The accelerator section length can be expressed in phase units as:

$$
\varphi=\frac{2 \pi L}{\beta_{\mathrm{ph}} \lambda}=\frac{2 \pi f}{\beta_{\mathrm{ph}} c} L
$$

And therefore:

$$
\frac{d \varphi}{d f}=\frac{2 \pi L}{\beta_{\mathrm{ph}} c}\left(1-\frac{f}{\beta_{\mathrm{ph}}} \frac{d \beta_{\mathrm{ph}}}{d f}\right)
$$

Or

$$
\Delta \varphi=\frac{2 \pi L}{\beta_{\mathrm{ph}} c}\left(1-\frac{f}{\beta_{\mathrm{ph}}} \frac{d \beta_{\mathrm{ph}}}{d f}\right) \Delta f
$$

In order to calculate the energy deviation $\delta \gamma=\left(\gamma_{\max }-\Delta \gamma\right) / \gamma_{\max }$, we rewrite Eq. (77) as:

$$
\delta \gamma=1-\frac{\sin \Delta \varphi}{\Delta \varphi} \approx \frac{(\Delta \varphi)^{2}}{6}=\frac{1}{6}\left[\frac{2 \pi L}{\beta_{p h} c}\left(1-\frac{f}{\beta_{p h}} \frac{d \beta_{p h}}{d f}\right) \Delta f\right]^{2}
$$

This allows us to estimate the effect of the RF frequency deviation on beam energy.

\section{Summary}

This tutorial review provided an in-depth review of the beam dynamics aspects of buncher design for industrial-grade low-to-medium energy electron linacs from fundamentals and theory to practical design tips. From ballistic pre-bunchers to constant and tapered velocity bunchers, various bunching techniques were covered. The phase space concept was introduced in this tutorial, which provides a clear and illustrative demonstration of the bunching process. Finally, the advanced practical topics such as energy spectrum quality, beam/structure interaction, transient processes, and influence of bunching on the acceleration process were demonstrated.

\section{Conclusion}

The complexity of accelerator design for industrial applications is usually underestimated. Although the beam quality and other parameters of such accelerators are not as extreme as those developed for scientific facilities, free-electron lasers or ion accelerators, industrial machines have to be commercially efficient. Therefore, besides the required parameters, the designer must account for the complexity and the cost efficiency of the accelerator. Moreover, some novel industrial accelerator application set requirements for rapid beam variability, system compactness or high throughput, which conventional machines cannot satisfy. In this view, the development of new efficient industrial accelerators is an important problem.

One of the key elements of an industrial accelerator is its frontend that includes the injection and bunching systems, which greatly define the accelerated beam parameters and linac performance in general. Unfortunately, most of the available textbooks on electron linac design 
and even some accelerator designers devote minimal attention to electron beam bunching, which can lead to low efficiency, low output, or low beam quality of the accelerated beam in improperly designed machines. We hope that the theory provided in the tutorial, the explanation of the phase portrait concept, and the detailed overview of different buncher concepts and techniques, along with practical guidance for their design, will allow the reader to design accelerators with improved parameters for the next generation of industrial applications.

Acknowledgments The material was inspired by the transactions of Prof. Oleg Anatolyevich Valdner from the Moscow Engineering-Physics Institute, which unfortunately have never been published in English and therefore are undeservedly unnoticed by international readers. The author would also like to express his acknowledgment to the following colleagues for important discussions on electron bunching and electron linacs design: Prof. Nikolai Sobenin, Prof. Eduard Masunov and other colleagues from Moscow EngineeringPhysics Institute (currently, National Research Nuclear University "MEPhI"), Dr. Alexander Zavadtsev and Dr. Dmitriy Zavadtsev from ScanTech Sciences (currently, Nano-Invest, Moscow, Russia), Dr. Alexander Plastun from the Facility for Rare Isotope Beams (USA), Dr. David Bruhwiler from RadiaSoft Inc. (USA) [130], Dr. Valery Dolgashev and Dr. Anatoly Krasnykh from SLAC National Accelerator Laboratory (USA), Prof. Valentin Paramonov from the Institute for Nuclear Research (Russia), Prof. Vasiliy Shvedunov from Moscow State University (Russia), Dr. Anatoly Gryzlov from NPO “Thoriy” (Russia), Dr. Alexei Smirnov from Viewray (USA), Dr. Yuri Batygin, Dr. Lloyd Young and Dr. James Potter from Los Alamos National Laboratory (USA), Dr. Timur Shaftan from Brookhaven National Laboratory (USA) as well as Mr. Salime Boucher, Dr. Alex Murokh and Dr. Anatoli Arodzero from RadiaBeam, USA [131]. Special thanks are due to Prof. Maria Gusarova (MEPhI, Russia) for providing me with rare literature and Mr. Konstanin Nikolski for a valuable feedback.

Open Access This article is licensed under a Creative Commons Attribution 4.0 International License, which permits use, sharing, adaptation, distribution and reproduction in any medium or format, as long as you give appropriate credit to the original author(s) and the source, provide a link to the Creative Commons licence, and indicate if changes were made. The images or other third party material in this article are included in the article's Creative Commons licence, unless indicated otherwise in a credit line to the material. If material is not included in the article's Creative Commons licence and your intended use is not permitted by statutory regulation or exceeds the permitted use, you will need to obtain permission directly from the copyright holder. To view a copy of this licence, visit http://creativecommons.org/licenses/by/4.0/.

\section{Appendix A: Beam compression in ballistic bunchers}

After passing the resonator, the electron's initial velocity $v_{\text {inj }}=\sqrt{\frac{2 q}{m} U_{\mathrm{DC}}}$ will change, depending on the time when it enters the RF field as:

$$
v=\sqrt{\frac{2 q}{m}\left(U_{\mathrm{DC}}+U_{\mathrm{RF}} \sin \omega_{\mathrm{RF}} t_{0}\right)}
$$

And the time, required for the particle to pass the drift space, can be calculated as:

$$
\Delta t=\frac{L}{\sqrt{\frac{2 q}{m} U_{\mathrm{DC}}\left(1+\frac{U_{\mathrm{RF}}}{U_{\mathrm{DC}}} \sin \omega_{\mathrm{RF}} t_{0}\right)}}
$$

The RF voltage of the cavity must be less than the injection voltage $U_{\mathrm{RF}}<U_{\mathrm{inj}}$. Otherwise, it will decelerate the particles too much, and some of them will be rejected back to the gun. In this case, using a relationship $\frac{1}{\sqrt{1+x}} \approx 1-\frac{x}{2}$ [132], we can approximate the expression (83) to:

$$
\Delta t=\frac{L}{v_{\text {inj }}}\left(1-\frac{U_{\mathrm{RF}}}{2 U_{\mathrm{DC}}} \sin \omega_{\mathrm{RF}} t_{0}\right)
$$


Here, the RF voltage $U_{R F}$ also includes the cavity's transit-time factor, which accounts for the RF phase change during the electron motion within the resonator [58]. By replacing time with phase $\left(\varphi=\frac{2 \pi t}{T_{\mathrm{RF}}}=\frac{2 \pi t c}{\lambda}\right)$, we can rewrite this equation as $\Delta \varphi=\varphi_{0}-r \cdot \sin \varphi_{0}$, where $r$ depends on the pre-buncher parameters and defines its compression efficiency, which can be calculated by using Eq. (34).

To estimate the percentage of particles located within the bunch, we need to find the original phase of the particle that arrived at the pre-buncher behind the crest $\varphi_{0}<-\pi / 2$ but now meets the most decelerated particle $\left(\varphi_{0}=\pi / 2\right)$ —point A in Fig. 18. This condition can be described as:

$$
\varphi_{A}-r \cdot \sin \varphi_{A}=\frac{\pi}{2}-r
$$

Similarly, for the particle that arrived ahead of the negative crest $\varphi_{0}>\pi / 2$ that reaches the most accelerated particle $\left(\varphi_{0}=-\pi / 2\right)$ we can write:

$$
\varphi_{B}-r \cdot \sin \varphi_{B}=-\frac{\pi}{2}+r
$$

And the bunched particle ratio will contain $k_{\mathrm{N}}=\left(\varphi_{\mathrm{B}}-\varphi_{\mathrm{A}}\right) / 2 \pi$ particles. Unfortunately, these equations are transcendent and do not have an analytical solution. Figure 19 presents the plot of numerically calculated bunch phase lengths $(\Delta \varphi)$ and number of particles in in the bunch $\left(k_{\mathrm{N}}\right)$ for different values of parameter $r$.

\section{Appendix B: Frequency of phase oscillations}

If the electrons' equilibrium phase is equal to $\pi / 2$, then expression (13) can establish the relationship between the particle's phase and energy. In other cases, more general expressions should be used. The speed of particle rotation along phase trajectories is defined by the frequency of phase oscillations, which can be calculated by combining Eqs. (9)-(13) in the form $\ddot{\varphi}+\Omega^{2} \sin \varphi=0$ and applying the small-amplitude approximations [7, 10, 25], which results in the following expression [23]:

$$
\Omega=\omega_{\mathrm{RF}} \sqrt{A \frac{\sqrt{\left(1-\beta_{\mathrm{ph}}^{2}\right)^{3}}}{2 \pi \beta_{\mathrm{ph}}} \sin \varphi_{s}}
$$

The dependence of this frequency on the RF field amplitude for different phase velocities is presented in Fig. 49 and demonstrates that the frequency of phase oscillations is comparable with the EM field frequency. The frequency of oscillations grows with the field amplitude, and for low phase velocities, it can become faster than the RF frequency. For the phase velocities close to the speed of light $\left(\beta_{\mathrm{ph}}=1\right)$, this frequency reduces to zero, which means that no phase oscillations occur in the pure acceleration regime. Equation (87) is valid in the assumption of small amplitude of phase oscillations. With an increase of oscillation amplitude, their frequency is reduced and approaches zero near the separatrix.

For an equilibrium phase of $\varphi_{\mathrm{s}}=\pi / 2$, the dependence of phase oscillation frequency on field amplitude can be defined by the coefficient [23]:

$$
K_{p h}=\sqrt{\cos \frac{\psi}{2}}
$$

where $\psi$ is the amplitude of phase oscillations. The expression assumes that phase oscillations are harmonic for all amplitudes. This assumption is valid for small amplitudes and is 


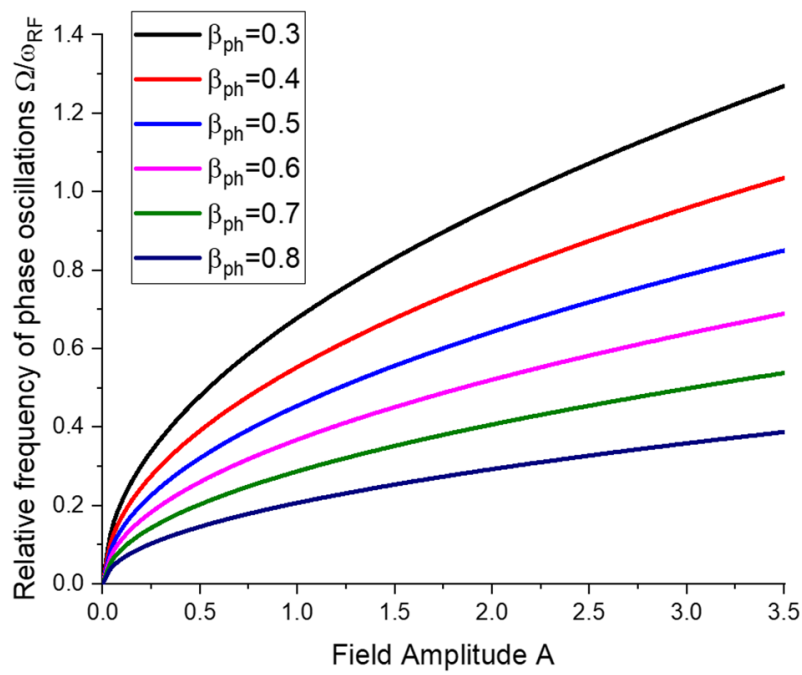

Fig. 49 Relative frequency of phase oscillation as a function of field amplitude and phase velocity

approximately valid for large amplitudes at phase velocities that are not very close to the speed of light. In the general case, $K_{\mathrm{ph}}$ is a complex function of multiple parameters, such as field amplitude A, phase velocity, and electron phase [24], since the instantaneous frequency in the top part of the trajectory is slower than for the lower part, as can be observed in Fig. 22. This relativistic effect is related to the considerable variation of electron's mass and velocity in these two regions. However, the correction (88) is usually sufficient. By combining (87) and (88), formula (35) can finally be derived.

\section{Appendix C: Phase and velocity spread in waveguide bunchers}

It is interesting to calculate when the maximum number of particles is bunched into the minimum phase interval. This happens when the particles, initially equidistant from both ends of the equilibrium phase $\left(\varphi_{1}=\pi / 2-\Delta \varphi_{0}\right.$ and $\varphi_{2}=\pi / 2+\Delta \varphi_{0}$ as shown in Fig. 22b), simultaneously reach the same phase $\varphi_{3}$ (as shown in Fig. 22c). For harmonic oscillations, the relationship between these phases can be calculated as [24]:

$$
\Delta \varphi=\frac{1}{2} \varphi_{1} \cos \left(\sqrt{\frac{\cos \frac{\varphi_{1}}{2}}{\cos \frac{\varphi_{2}}{2}}} a \cos \frac{\varphi_{3}}{\varphi_{1}}\right)
$$

The numerical solution to this equation is presented in Fig. 19 (blue line).

Now we find the length of a bunching section $\left(L_{\mathrm{b}}\right)$ required to compress the bunch into a specific phase width. This length is related to the equilibrium particle's time-of-flight $\left(t_{0}\right)$ as $\mathrm{L}=t_{0} \cdot \beta_{\mathrm{ph}} \cdot \mathrm{c}$. By applying Eq. (35), we can find the time of flight for a particle with initial phase $\varphi_{2}$. This time will be equal to the phase shift from $\varphi_{2}$ to $\varphi_{3}$, obtained by a combination of (35) and (89):

$$
t_{2}=\frac{a \cos \frac{\varphi_{3}}{\varphi_{2}}}{\omega_{\mathrm{RF}} \Omega K_{\mathrm{ph}}}
$$


A particle with initial phase $\varphi_{2}$ will enter the bunching section earlier than the equilibrium particle by the time $\Delta t_{2}=\varphi_{2} / \omega_{\mathrm{RF}}$, and will exit later by $\Delta t_{3}=\varphi_{3} / \omega_{\mathrm{RF}}$. Therefore, the time $t_{2}$, defined by Eq. (90), is larger than $t_{0}$ by the value of $\Delta t=\Delta t_{2}-\Delta t_{3}=\omega_{R F}\left(\varphi_{2}-\varphi_{3}\right)$. Finally, by combining these relations, it is possible to calculate the required length of the buncher:

$$
\frac{L_{b}}{\lambda}=\frac{\beta_{\mathrm{ph}}}{2 \pi}\left(\frac{r}{\Omega}-\varphi_{2}+\varphi_{3}\right)
$$

Here the parameter $r$ has a similar meaning as for pre-bunchers and is defined as [23]:

$$
r=\frac{a \cos \frac{\varphi_{3}}{\varphi_{2}}}{\sqrt{\cos \frac{\varphi_{2}}{2}}}
$$

Its values can be found using Fig. 19 (blue line), while $\Omega$ can be found from (35).

During the electron bunching process, along with phase compression, energy spread also develops. The highest energy spread is observed for particles with initial phases close to 0 and $\pi$. It can be estimated by the maximum and minimum values of the energies on the top and bottom points of the separatrix at $\pi / 2$ using formulas (13) and (17):

$$
\frac{1-\beta_{\mathrm{ph}} \beta}{\sqrt{1-\beta^{2}}}-\frac{A \beta_{\mathrm{ph}}}{2 \pi}=\sqrt{1-\beta_{\mathrm{ph}}^{2}}+\frac{A \beta_{\mathrm{ph}}}{2 \pi}
$$

It is convenient to introduce the parameter $a$ as:

$$
a=\sqrt{1-\beta_{\mathrm{ph}}^{2}}+\frac{A \beta_{\mathrm{ph}}}{2 \pi}
$$

So that Eq. (93) takes the form of:

$$
1-\beta_{\mathrm{ph}} \beta=a \sqrt{1-\beta^{2}}
$$

which is now can be solved for $\beta$ :

$$
\beta_{1,2}=\frac{\beta_{\mathrm{ph}} \pm \sqrt{\beta_{\mathrm{ph}}^{2}+\left(a^{2}-1\right)\left(a^{2}+\beta_{\mathrm{ph}}^{2}\right)}}{a^{2}+\beta_{\mathrm{ph}}^{2}}
$$

The difference between the two values of $\beta$ characterizes the energy spread at the exit of the buncher.

Similarly, we can calculate the buncher length and the energy spread for a buncher with $\beta_{\text {ph }}>\beta_{\text {inj. }}$. First, we will calculate when the particle with the initial phase $\varphi=\pi / 2$ returns to the same phase after half a period of phase oscillations (Fig. 23c). By using Eq. (13), written for the $\varphi=\pi / 2$ :

$$
H_{1}=\frac{1-\beta_{\mathrm{ph}} \beta}{\sqrt{1-\beta^{2}}}-\frac{A \beta_{\mathrm{ph}}}{2 \pi}
$$

Considering Eq. (87) for the phase oscillation amplitude of $\psi=\varphi_{2}-\pi / 2$, it is easy to calculate the latter:

$$
\psi=a \cos \left[\frac{2 \pi}{A \beta_{\mathrm{ph}}}\left(\sqrt{1-\beta_{\mathrm{ph}}^{2}}-\frac{1-\beta_{\mathrm{ph}} \beta_{\mathrm{inj}}}{\sqrt{1-\beta_{\mathrm{inj}}^{2}}}\right)+1\right]
$$


The time of flight for the considered particle is half of the phase oscillation period and is equal to $t_{1}=\frac{T}{2}=\frac{\pi}{\omega_{\mathrm{RF}} \omega_{\mathrm{ph}}}$, and the buncher length can be calculated as:

$$
\frac{L_{b}}{\lambda}=\sqrt{\frac{\pi \beta_{\mathrm{ph}}^{2}}{2 A \cos \frac{\psi}{2}\left(1-\beta_{\mathrm{ph}}^{2}\right)^{3 / 2}}}
$$

The same methodology allows us to calculate the energy gain of this particle as we used for (96):

$$
\begin{gathered}
\beta_{\text {out }}=\frac{\beta_{\mathrm{ph}}+\sqrt{\beta_{\mathrm{ph}}^{2}+\left(b^{2}-1\right)\left(b^{2}+\beta_{\mathrm{ph}}^{2}\right)}}{b^{2}+\beta_{\mathrm{ph}}^{2}} \\
b=\frac{1-\beta_{\mathrm{ph}} \beta_{\mathrm{inj}}}{\sqrt{1-\beta_{\mathrm{inj}}^{2}}}
\end{gathered}
$$

\section{Appendix D: Parameters choice in Lomnev's buncher design approach}

Let us demonstrate the optimization process with an example of a low-power buncher design with DC injection at $50 \mathrm{keV}$. The starting phase velocity is chosen to match this energy $\left(\beta_{\mathrm{ph}}\right.$ $=0.413$ ) and the amplitude $A_{\mathrm{M}}=0.52$. In this case, the middle section is within the 15 to $30 \mathrm{~cm}$ range, with the whole buncher length of $60 \mathrm{~cm}$. Figure 50 provides the dependences of phase and energy spread on the coefficients $m$ and $n$.

These results can be explained by looking at Eqs. (9) and (10). The value of $\beta_{\text {ph }}$ defines the intensity of phase oscillations and the rate of their attenuation. It can be seen from Eq. (48) and Fig. 50 that for fast changes of velocity $\left(\partial \beta_{\mathrm{ph}} / \partial \mathrm{z}\right)$, the oscillations quickly decay, and the particles start to accelerate in an under-bunched configuration. If the accelerator length is small, this strategy leads to large bunches with relatively small energy spread. However, this method is very approximate. Figure 50, left, shows that there is a region for $\beta_{\text {ph }}$ variation where $\Delta \varphi$ is minimal. In this case, the phase velocity changes such that the particle with the initial phase of $\varphi=\pi$ shifts below the equilibrium phase at the middle section entrance and above the equilibrium at its end. Another observation is that phase oscillations' amplitude also decays with amplitude growth $(\partial \mathrm{A} / \partial \mathrm{z})$. The amplitude variation changes the energy spread more than the phase length, as shown in Fig. 50, left. In practice, the optimal $\mathrm{n}$ is chosen to achieve the shortest phase length, and then $\mathrm{m}$ is optimized to improve the energy spread.

Finally, the initial section of the buncher (I) can be designed. This section plays the most significant role in the beam bunching, so the relationship between $\partial \beta_{\mathrm{ph}} / \partial \mathrm{z}$ and $\partial \mathrm{A} / \partial \mathrm{z}$ is significant. For slow amplitude variation, phase bunching dominates the energy bunching. The opposite scenario is realized for considerable amplitude variation. A reasonably uniform phase and energy spread can be achieved for $\mathrm{n}$ and $\mathrm{m}$, corresponding to the phase trajectories' maximal stability during the capture. Such an initial part will provide the best initial conditions for the following sections.

To study the influence of the phase velocity and amplitude variation, we provide the results of beam dynamics simulations [26] with the buncher parameters designed according to Eqs. (48), (49), and for the same linac that was described above. Both the middle and end sections have the same parameters $n=0.25, m=0.11$. The results of these studies are presented in Fig. 51. These plots allow making conclusions similar to those for the middle 

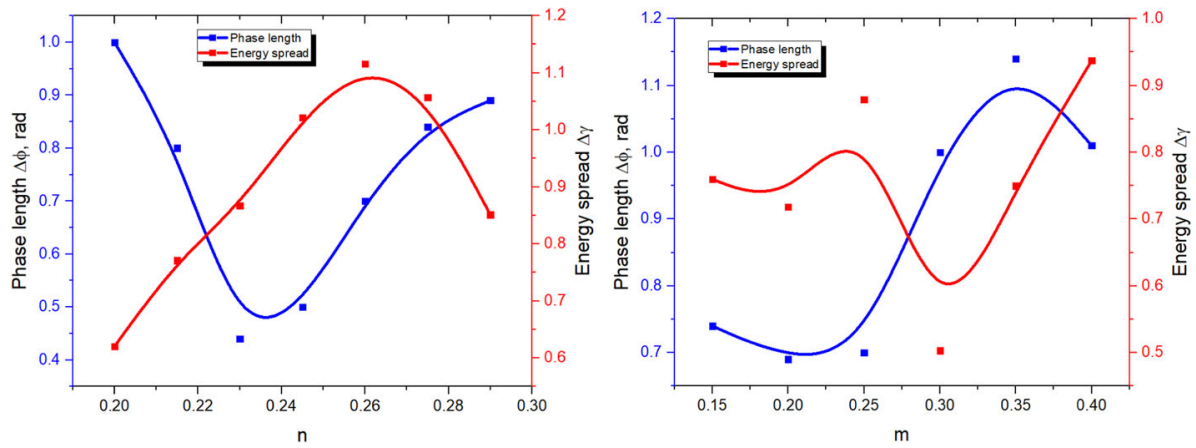

Fig. 50 Dependence of phase length (blue) and energy spread (read) on $n$ and m parameters in Eqs. (48), (49) for the middle section design. Left: $\mathrm{m}=0.11$. Right: $\mathrm{n}=0.25$. In both cases $k 1=0.6435, k 2=0.7126$
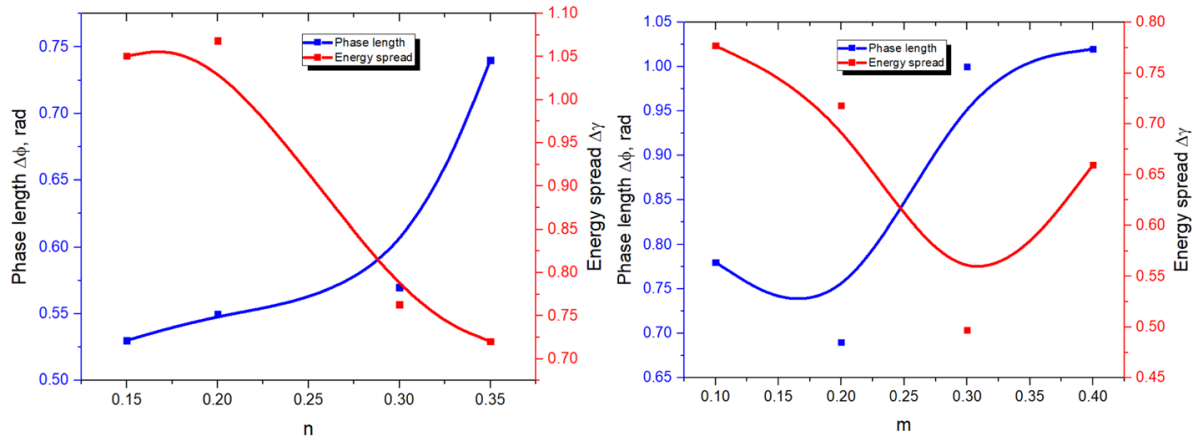

Fig. 51 Phase length (blue) and energy spread (read) dependence on $\mathrm{n}$ and $\mathrm{m}$ parameters in Eqs. (46), (47) for the initial section design. Left: $\mathrm{m}=0.11$. Right: $n=0.25$. In both cases $k 1=0.6435, k 2=0.7126$

section: small variations of $\beta_{\mathrm{ph}}(\mathrm{z})$ lead to short phase length of the bunch, while large phase velocity variations lead to smaller energy spread. Since the velocity variation range in the initial part should be small to provide the maximum capture, it is more important to choose the proper amplitude variation $\mathrm{A}(\mathrm{z})$, since this function will influence the bunching process in all following buncher sections. A sharp amplitude change leads to elongation of the bunch (large $\Delta \varphi$ ).

Since the phase velocity changes slowly in the buncher's initial part, the outmost particles with the phases $-\pi / 2$ and $3 \pi / 2$ and the particles near $\pi / 2$ have amplitude and phase variations close to zero. Therefore, it is not reasonable to increase the field amplitude until these particles exit these phase regions. Due to the limited range of possible amplitudes in a DLS structure, defined by the available RF power, it is reasonable to start amplitude variation at a point when the electron with the initial phase $\varphi=3 \pi / 2$ reaches a phase slightly exceeding $\pi / 2$. A further variation of $\mathrm{A}(\mathrm{z})$ should be performed in connection with phase velocity variation $\beta_{\text {ph }}(\mathrm{z})$ according to Eqs. (48), (49).

It is also worth mentioning the influence of the initial amplitude on the bunching efficiency, as demonstrated in Fig. 52. In this case, the phase velocity profile remained the same for all cases, and the amplitude linearly changed from $\mathrm{A}(0)$ at the beginning to $A=0.52$ at $20 \mathrm{~cm}$. This plot demonstrates that the broader range of amplitude variation improves the bunching properties of the buncher. For smaller initial values of the amplitude, the energy spread becomes smaller. Smaller amplitudes are also beneficial for the capture ratio. Simultaneously, 


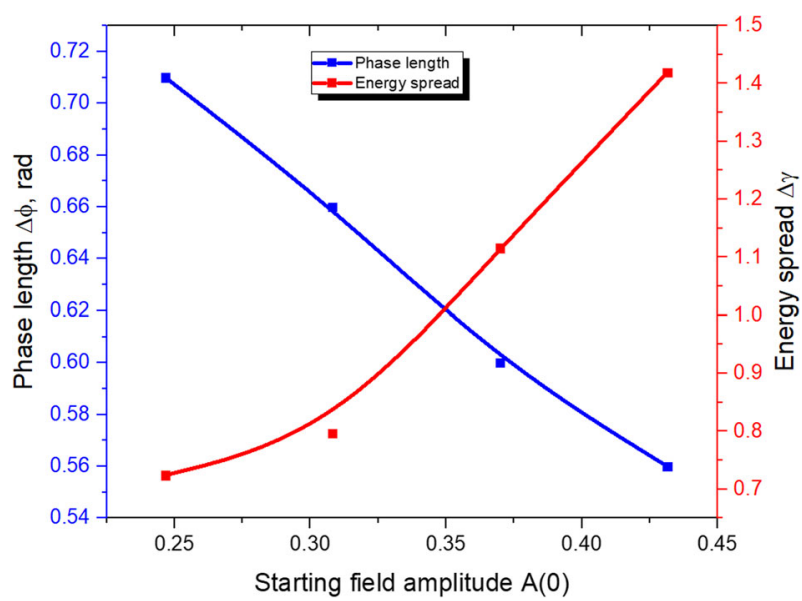

Fig. 52 Dependence of phase length (blue) and energy spread (red) on the field amplitude at the beginning of the bunching section (I) $\mathrm{A}(z=0)$

operation at low-fields does not increase the overall buncher length since it is possible to move the phase oscillations to the region of smaller $\varphi$, where the decay is faster.

\section{Appendix E: Phase shift caused by the beam loading effect}

If the bunches are not located on the crest of the accelerating wave, there will be a phase difference between this field and the wake field, which will result in a change in the phase velocity of the resulting wave. This case is illustrated in Fig. 53 as a representation of the field vector sum (called phasor). Here $\overrightarrow{E_{\mathrm{eff}}}=\overrightarrow{E_{\mathrm{RF}}}+\overrightarrow{E_{\mathrm{beam}}}$ or $E_{\mathrm{eff}} e^{i \psi}=E_{\mathrm{RF}} e^{i \varphi}-E_{\text {beam }}$. Since the wake field phase always follows the beam, its complex amplitude will always have a negative real value. This plot demonstrates that beam loading leads to a phase shift of the effective accelerating field by a value of $\chi=\psi-\varphi$.

Since $E_{\mathrm{RF}}(z)=E_{\mathrm{RF}}(0) e^{-\alpha z}$ and $\frac{d E_{\mathrm{RF}}}{d z}=-\alpha E_{\mathrm{RF}}$, it is possible to write the equation for the effective field as:

$$
\frac{d E_{\mathrm{eff}}}{d z}=-\alpha\left(E_{\mathrm{eff}}+I \cdot r_{\mathrm{sh}} \cos \psi\right)
$$

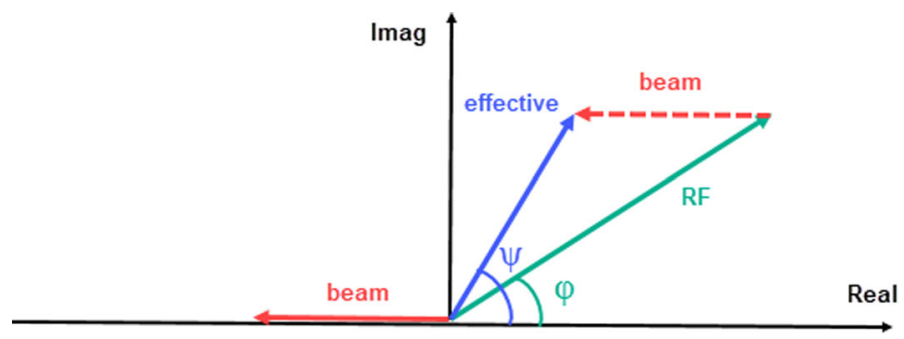

Fig. 53 Vector diagram (phasor) of accelerating (blue), wake (red) and the resulting (black) fields. $\varphi$ corresponds to the phase of the RF wave, relative to the bunch, and $\psi$ to the resulting wave. The wake field phase is always decelerating and follows the bunch center 


$$
E_{\mathrm{eff}} \frac{d \psi}{d z}=\alpha \cdot I \cdot r_{\mathrm{sh}} \sin \psi
$$

By combining these two equations, it is possible to write the following differential equation:

$$
\frac{\frac{d^{2} \psi}{d z^{2}}}{\frac{d \psi}{d z}}-2 \cot \psi \cdot \frac{d \psi}{d z}=\alpha
$$

which can be integrated according to the initial conditions $E_{\mathrm{eff}}(0)=E_{\mathrm{RF}}(0)=E_{0}$ and $\psi(0)=\varphi$ :

$$
\frac{d \psi}{d z}=\frac{\alpha r_{\mathrm{sh}} I_{b}}{E_{0}} \cdot \frac{\sin ^{2} \psi}{\sin \varphi} e^{\alpha z}
$$

This demonstrates that the effective phase $\psi$ is not constant during acceleration, even when the phase of the RF field is constant. Since $\mathrm{d} \psi / \mathrm{dz}$ is proportional to the beam current, this value is called phase slippage due to beam loading. This phase slippage is different from that caused by the difference in phase and particle velocities that was considered in Chapter 2 . The effect of beam loading phase slippage can be converted to an equivalent change of phase velocity. To do that, we need to rewrite Eq. (8) as:

$$
\frac{d \psi}{d z}=k\left(\frac{1}{\beta_{\mathrm{ph}}+\Delta \beta_{\mathrm{ph}}}-\frac{1}{\beta}\right) \approx k\left(\frac{1}{\beta_{\mathrm{ph}}}-\frac{1}{\beta}-\frac{\Delta \beta_{\mathrm{ph}}}{\beta_{\mathrm{ph}}^{2}}\right)=-k \frac{\Delta \beta_{\mathrm{ph}}}{\beta_{\mathrm{ph}}^{2}}
$$

Since the phase velocity of the wake field is equal to the beam velocity as discussed above. Here $\mathrm{k}$ is the wave number. By comparing (105) and (106), we can write the expression for the effective phase velocity change due to beam loading:

$$
\frac{\Delta \beta_{p h}}{\beta_{p h}^{2}}=-\frac{\alpha r_{s h} I_{b}}{k E_{0}} \cdot \frac{\sin ^{2} \psi}{\sin \varphi} e^{\alpha z}
$$

This equation can be integrated with the same initial conditions to obtain the function of the phase change:

$$
\cot \psi=\cot \varphi-\frac{I_{b} \cdot r_{\mathrm{sh}}}{E_{0} \cdot \sin \varphi}\left(1-e^{\alpha z}\right)
$$

If $\varphi \neq 0$ the phase slippage can eventually reach the value $\psi=\pi / 2$, which defines the length of the accelerating section after which acceleration ceases. For lengths beyond this value, the beam will slip into decelerating phase. This length can be obtained by applying $\psi$ $=\pi / 2$ in Eq. (108):

$$
L_{\max }=\frac{1}{\alpha} \ln \left(1+\frac{E_{0} \cdot \cos \varphi}{I_{b} \cdot r_{s h}}\right)
$$

The existence of a maximum length means the beam can reach only a limited voltage gain in such an accelerating section and that a part of the RF power will always be unused and will go into the load. This unused part of the RF power can be found as [23]:

Therefore, a field approach allows more accurate description of the processes in accelerating structures due to beam loading: the equivalent phase velocity change, phase slippage, power efficiency, and transient behavior. Similar effects occur due to the RF frequency change.

$$
P_{\text {load }}=\frac{1}{2 \alpha \cdot r_{\mathrm{sh}}} E_{\mathrm{eff}}^{2}\left(L_{\mathrm{max}}\right)=\frac{E_{0}^{2} \cdot \sin ^{2} \varphi}{2 \alpha \cdot r_{\mathrm{sh}}\left[1+\frac{E_{0} \cdot \cos \varphi}{I_{b} \cdot r_{\mathrm{sh}}}\right]^{2}}=\frac{P_{\mathrm{RF}} \cdot \sin ^{2} \varphi}{\left[1+\sqrt{\frac{2 \alpha \cdot P_{\mathrm{RF}}}{r_{\mathrm{sh}}}} \frac{\cos \varphi}{I_{b} \cdot}\right]^{2}}
$$




\section{References}

1. S.V. Kutsaev, Advanced technologies for applied particle accelerators and examples of their use (Review). Tech. Phys. 66(2), 161-195 (2021)

2. L. Evans, P. Bryant, LHC Machine. J. Instrum. 3, S08001 (2008)

3. W. Barletta, T. Feder, Accelerator school travels university circuit. Phys Today 63(2), 20 (2010)

4. S. Boucher, S. Kutsaev, A. Murokh, APS Division of Physics of Beams, Annual Newsletter (APS Physics Publishing, USA, 2019), p. 24

5. R. Hellborg, Electrostatic Accelerators: Fundamentals and Applications (Springer, 2005).

6. K. Takayama, R.J. Briggs, Briggs Induction Accelerators (Springer, 2011).

7. I.M. Kapchinskij, Theory of Linear Resonant Accelerators (Energoizdat, Moscow, 1982), p 240 (in Russian)

8. A. Seriy, "Unifying Physics of Accelerators, Lasers and Plasma (CRC Press, 2016).

9. J.B. Rosenzweig, Fundamentals of Beam Physics (Oxford University Press, 2003).

10. T.P. Wangler, RF Linear Accelerators, 2nd edn. (Wiley, 2008).

11. G. Floquet, Sur les équations différentielles linéaires à coefficients périodiques. Annales de l'École Normale Supérieure 12, 47-88 (1883). (in Russian)

12. J.W. Wang, RF properties of periodic accelerating structures for linear colliders. Ph.D. Thesis, Stanford University, 1989.

13. https://www.3ds.com/products-services/simulia/products/cst-studio-suite/

14. V.I. Kaminsky, M.V. Lalayan, N.P. Sobenin, Accelerating Structures (MEPHI, Moscow, 2005). (in Russian)

15. P.M. Lapostolle, A.L. Septier, Septier Linear Accelerators (Elsevier, 1970).

16. D. Tronc, Traveling wave acceleration of protons. Nucl. Instrum. Methods Phys. Res. Sect. 327, 253 (1993)

17. S.V. Kutsaev et al., High-gradient low- $\beta$ accelerating structure using the first negative spatial harmonic of the fundamental mode. Phys. Rev. Accel. Beams 20, 120401 (2017)

18. A. Durkin, A. Kolomiets, V. Paramonov, Investigation of the second harmonic influence on focusing of moderate energy protons in travelling wave accelerating structure. J. Phys. Conf. Ser. 1686, 012069 (2020)

19. S.V. Kutsaev et al., Design of hybrid electron linac with standing wave buncher and traveling wave structure. Nucl. Instrum. Meth. Phys. Res. A 636(1), 13-30 (2011)

20. I.N. Karetnikov, V.A. Slivkov, V.A. Teplyakov et al., Linear Accelerators of Ions (Gosatomizdat, Moscow, 1962). (in Russian)

21. S. Hanna, RF Linear Accelerators for Medical and Industrial Applications (Artech House, 2012).

22. H. Wiedemann, Particle Accelerator Physics, 4th edn. (Springer, 2015).

23. O.A. Valdner, A.D. Vlasov, A.V. Shalnov, Linear Accelerators, Atomizdat, 1969 (in Russian)

24. O.A. Valdner, Linear Accelerators of Electrons, Atomizdat, 1966 (in Russian)

25. A.N. Lebedev, A.V. Shalnov, Fundamental of Accelerator Physics and Technology, 2nd edn. (Energoatomizdat, 1991). (in Russian)

26. G.A. Tyagunov 1960 Accelerators", Atomizdat, 1960 (in Russian).

27. O.S. Milovanov, N.P. Sobenin, RF Technology. Atomizdat, 1980. (in Russian),

28. A.S. Roshal, "Modelling of charged particle beams", Atomizdat, 1979 (in Russian).

29. E.L. Burshtein, G.V. Voskresenskiy, Electron linear accelerators with intensive beams, Atomizdat, 1970 (in Russian)

30. M.V. Arsentyeva, K.N. Berdnikov, A.E. Levichev, Analysis of the particle longitudinal motion during the injection process into the disk loaded traveling wave accelerating structure. J Instrum 15, P10015 (2020)

31. M. Stanley Livingston, High Energy Accelerators (Interscience Publishers, 1954).

32. M. Stanley Livingston, J.B. Blewett, Blewett Particle Accelerators (Mc Graw Hill Book Company Inc, 1992).

33. H. Bruck, Accelerateurs Circulaires de Particules (PUF, Paris, 1966). ((in French))

34. E. Persico, E. Ferrari, S.E. Segre, Principles of Particles Accelerators (W.A. Benjamin, Inc., 1968).

35. A.D. Vlasov, Theory of Linear Accelerators (Programm for scientific translations, Jerusalem, 1968).

36. M. Conte, W.W. Mac Kay, An Introduction to the Physics of Particle Accelerators (World Scientific, 1991).

37. D.A. Edwards, M.J. Syphers, An Introduction to the Physics of High Energy Accelerators (Wiley, 1993).

38. M. Reiser, Theory and Design of Charged Particles Beams (Wiley, 1994).

39. K. Wille, The Physics of Particle Accelerators: An Introduction (Oxford University Press, 2000).

40. E.J.N. Wilson, An Introduction to Particle Accelerators (Oxford University Press, 2001). 
41. O.A. Valdner, N.P. Sobenin, B.V. Zverev, I.S. Schedrin, Corrugated Waveguides: Reference Book (Energoatomizdat, 1991). (in Russian)

42. R. Agustsson, S. Boucher, S. Kutsaev, Split structure particle accelerators, International Patent Application WO 2018222839A1 (2018).

43. R. Agustsson, S. Boucher, S. Kutsaev, Modified split structure particle accelerators, International Patent Application WO 2020061204A1 (2020).

44. C.J. Karzmark, C.S. Nunan, E. Tanabe, Medical Electron Accelerators (McGraw-Hill Incorporated, Health Professions Division, 1993).

45. A.V. Alyamovski, Electron Beams and Electron Guns (Soviet Radio, Moscow, 1966). (in Russian)

46. B.S. Ishkhanov, A.V. Poseryaev, V.I. Shvedunov, Estimating the charged particle beam parameters in view of the space charge forces. Instrum Exp Tech 48(6), 747 (2005)

47. C.D. Child, Discharge From Hot CaO. Phys. Rev. (Series I) 32, 492 (1911)

48. A.D. Sushkov, Vacuum Electronics. Physical and Technical Fundamentals (Lan' Publishing, St. Petersburg, 2004). (in Russian)

49. V.I. Shvedunov, Electron accelerators design and construction at Lomonosov Moscow State University. Radiat Phys Chem 159, 95-100 (2019)

50. D. Bhattacharjee et al., Development of electron guns for linacs and DC accelerator. J. Phys. Conf. Ser. 390, 012071 (2012)

51. BYu. Bogdanovich et al., Distant Radiation Control with Linear Accelerators, vol. 1 (Energoatomizdat, Moscow, 2012).

52. A.N. Ermakov et al., Commissioning of High Efficiency Standing Wave Linac for Industrial Applications. In: Proc. RuPAC2016, St. Petersburg, Russia p.WECDMH01, 2016.

53. L. Ovchinnikova et al., Design of C-Band Electron Linear Accelerator for a Complex of Radiation Therapy. In: Proc. LINAC2018, Beijing, p.550, China, 2018.

54. O.A. Valdner, E.G. Pyatnov, V.D. Seleznev, Linear accelerator of electrons U-27 to the energy of 10 MeV. At. Energy 23(5), 465 (1967). (in Russian)

55. S.V. Kutsaev et al., Generalized 3D beam dynamics model for industrial traveling wave linacs design and simulations. Nucl. Instrum. Meth. Phys. Res. A 906, 127-140 (2019)

56. I.M. Kapchinskij, Dynamics of Particles in Linear Resonant Accelerators (Atomizdat, Moscow, 1966). (in Russian)

57. W.C. Hahn, A new method for the calculation of cavity resonators. J. Appl. Phys. 12, 62 (1941)

58. N.P. Sobenin, B.V. Zverev, Electrodynamic Characteristics of Accelerating Cavities (CRC Press, 1999).

59. Q. Chen et al., Design of RF chopper system for improving beam quality in FEL injector with thermionic gun. Nucl Instrum Meth Phys Res A 755, 78-84 (2014)

60. S.V. Kutsaev et al., Electron Accelerators for Novel Cargo inspection methods. Phys. Proc. 90, 115-125 (2017)

61. A.A. Zavadtsev et al., A dual-energy linac cargo inspection system. Instrum. Exp. Tech. 54, 241-248 (2011)

62. S.V. Kutsaev et al., X-ray sources for adaptive radiography and computed tomography. AIP Conf Proc 2160, 050014 (2019)

63. S.V. Kutsaev et al., Linear accelerator for security, industrial and medical applications with rapid beam parameter variation. Radiat Phys Chem 183, 109398 (2021)

64. C. Adolphsen et al., Conceptual Design for the New RPI 2020 Linac. SLAC-PUB-16137, 2014. https:// doi.org/10.2172/1344166

65. K. Whitham, Design of a 3 ampere peak steady state, 0.001 Duty, $10 \mathrm{MeV}$ linear electron accelerator. IEEE Trans Nucl Sci 14(3), 307 (1967)

66. A.S. Gilmour, Klystrons, Traveling Wave Tubes, Magnetrons, Crossed-Field Amplifiers, and Gyrotrons (Artech, NY, 2011).

67. A.S. Gilmour, Microwave and Millimeter-Wave Vacuum Electron Devices: Inductive Output Tubes, Klystrons, Traveling-Wave Tubes, Magnetrons, Crossed-Field Amplifiers, and Gyrotrons (Artech, 2020).

68. S. Kutsaev et al., Four-harmonic buncher for radioactive and stable beams switching at the ATLAS facility. Nucl Instrum Methods Phys Res A 905, 149-159 (2018)

69. P.N. Ostroumov et al., Heavy ion beam acceleration in the first three cryomodules at the Facility for Rare Isotope Beams at Michigan State University. Phys. Rev. Accel. Beams 22, 040101 (2019)

70. A.S. Plastun et al., Longitudinal Beam Dynamics in FRIB and ReA Linacs. In: Proc. 13th Int. Computational Accelerator Physics Conf. ICAP2018, Key West, FL, USA, p.330, 2018

71. M. Dal Forno et al., RF breakdown measurements in electron beam driven $200 \mathrm{GHz}$ copper and coppersilver accelerating structures. Phys. Rev. Accel. Beams 19, 111301 (2016)

72. M.A.K. Othman et al., Experimental demonstration of externally driven millimeter-wave particle accelerator structure. Appl. Phys. Lett. 117, 073502 (2020) 
73. S.V. Kutsaev et al., Nanosecond rf-Power Switch for Gyrotron-Driven Millimeter-Wave Accelerators. Phys. Rev. Appl. 11, 034052 (2019)

74. S.V. Kutsaev et al., Optical Spectrometer with a Pulse-to-Pulse Resolution for Terahertz and mm-wave Signals. IEEE Trans Terahertz Sci Technol 11(3), 2021.

75. M. Comunian et al., The IFMIF-EVEDA RFQ: Beam dynamics design. In: Proceedings of LINAC08, Victoria, BC, Canada, MOP036, 2008, pp. 3536-3538

76. G. Mavrogenes, T. Khoe, W. Wesolowski, Space charge effects in high current linear accelerator transport systems. IEEE Trans Nucl Sci 20(3), 478 (1973)

77. J. Haimson, Electron bunching in travelling wave linear accelerators. Nucl Istrum Meth 39(1), 13 (1966)

78. A.A. Zavadtsev et al., Complexes for the Inspection of the Elemental Composition of Cargo Based on Linear Electron Accelerators (NRNU MEPhI, Moscow, 2011). (in Russian)

79. S.V. Kutsaev et al., Band electron Linac for radiotherapy and security applications. Radiat. Phys. Chem. 185, 109494 (2021)

80. L. Young and J. Billen, The Particle Tracking Code PARMELA. In: Proc. of the 2003 Particle Accelerator Conference, p. 2531, 2003

81. K. Flottmann, S.M. Lidia and P. Piot, Recent Improvements to the ASTRA Particle Tracking Code. In: Proc. of PAC'03, Oregon, p. 3500, 2003

82. M.J. de Loos, S.B. van der Geer General Particle Tracer: A New 3D Code For Accelerator and Beam Line Design. In: Proc. 5th Eur. Part. Acc. Conf., Sitges, p.1241, 1996

83. V.N. Aseev et al., TRACK : The new beam dynamics code. In: Proc. of PAC05, Knoxville, Tennessee, 2005

84. F. Wolfheimer Latest Developments in 3D Charged Particle Simulations. In: presented at Int. Comp. Accel. Phys. Conf., 2012: http://epaper.kek.jp/ICAP2012/talks/moaai2_talk.pdf

85. A.J. Woods, L.D. Ludeking and D. Rhoades Performance Enhancement of FDTD-PIC Beam-Wave Simulations Using Multi-core Platforms. In: Proc. of PIER, Xi'an, 2010

86. S.V. Kutsaev, Electron dynamics simulations with Hellweg 2D code. Nucl. Instrum. Meth. Phys. Res. A 618(1-3), 298-305 (2010)

87. E.S. Masunov, Bunching and acceleration of the intensive beam in the waveguide accelerating structure with a presence of an external magnetic field. J. Tech. Phys. 49, 1462-1463 (1979). (in Russian)

88. P. M. Lapostolle Effets de la charge d'espace dans un accélérateur linéaire 'a protons. CERN, Geneva, Tech. Rep. CERN-AR-Int-SG-65-15, 1965 (in French).

89. S.V. Kutsaev et al., Cloud-based design of high average power traveling wave linacs. J. Physics: Conf. Series 941, 012106 (2017)

90. Y. Eidelman, S.V. Kutsaev and D.L. Bruhwiler Ellipsoid Space Charge Model for Electron Beam Dynamics Simulations. Phys. Part. Nuclei 52(3), 2021, in press

91. S.V .Kutsaev et al., Design, Simulations and Experimental Demonstration of an Intra-Pulse RampedEnergy Travelling Wave Linac for Cargo Inspection. In: Proc. 2nd North American Particle Accelerator Conference (NAPAC2016), Chicago, USA, p. TUPOA68 2016

92. The Hellweg repository on GitHub, https://github.com/radiasoft/rslinac

93. R. Warnecke, P. Guénard, Les tubes électroniques à commande par modulation de vitesse, vol 1 (GauthierVillars, Paris, 1951), 792p. (in French)

94. J. Haimson, Some aspects of electron beam optics and X-ray production with the linear accelerator. IEEE Trans. Nucl. Sci. 9, 32 (1962)

95. S.V. Kutsaev et al., Electron Linac with Deep Energy Control for Adaptive Rail Cargo Inspection System. 2015 In: IEEE Nuclear Science Symposium and Medical Imaging Conference (NSS/MIC), San Diego, CA, 2015

96. A. Arodzero et al., High speed, low dose, intelligent X-ray cargo inspection. In: 2015 IEEE Nuclear Science Symposium and Medical Imaging Conference (NSS/MIC), San Diego, CA, 2015

97. P. Lapostolle, "Introduction a 1'etude des accelerateurs lineaires (Introduction to the design of linear accelerators)", Course given at the INSTN, Saclay (France) CERN report 1966-20, 1966. (in French)

98. E. Leiss, Injection Systems for Linear Electron Accelerators (National Bureau of Standards, Washington, D.C., 1959).

99. D. Dupen, The story of Stanford's Two-Mile-Long Accelerator, SLAC-62, Technical Report, (1966)

100. E. Tanabe, R.W. Hamm, Compact multi -energy electron linear accelerators. Nucl Instrum Methods Phys Res Sect B 10, 871 (1985)

101. D. Baillie, B. Gino Fallone, S. Steciw, Design and simulation of a short, variable-energy 4 to $10 \mathrm{MV}$ S-band linear accelerator waveguide. Med. Phys. 44(6), 2124-2017 (2017)

102. S. Boucher et al., Compact, Inexpensive X-Band Linacs as Radioactive Isotope Source Replacements. In : Proc. IPAC2013, Shanghai, China, pp. THPWA051, (2013) 
103. S.V. Kutsaev et al., Sub-MeV ultra-compact linac for radioactive isotope sources replacement, nondestructive testing, security and medical applications. Nucl. Instrum. Meth. Phys. Res. B 159, 179-187 (2019)

104. M.F. Reusch and D.L. Bruhwiler, High-order space charge effects using automatic differentiation. In: AIP Conf. Proc. Vol. 391, p. 179, (1997)

105. S.P. Lomnev, Methods of Linear Accelerators Design (USSR Academy of Sciences, Moscow, 1962). (in Russian)

106. A.V. Shalnov, S.P. Lomnev, Preliminary Electron Bunching with a Klystron Resonator (MEPhI Press, Moscow, 1959). (in Russian)

107. O.A. Valdner, N.P. Sobenin, B.V. Zverev, I.S. Schedrin, Reference Book on Corrugated Waveguides, 2nd edn. (Atomizdat, Moscow, 1977). (in Russian)

108. V.N. Golovin, A.P. Ter-Saakov, The questions of extremal calculation of waveguide bunchers. In: Accelerators, vol. XII, ed. by O.A. Valdner (Atomizdat, Moscow, 1970), p.102. (in Russian)

109. V.N. Nalimov, N.A. Chernova, Statistical Methods for Planning of Extremal Experiments (Nauka, Moscow, 1965). (in Russian)

110. A. Sen, M. Srivastava, Regression Analysis—Theory, Methods, and Applications (Springer, Berlin, 2011).

111. B. Malakooti, Operations and Production Systems with Multiple Objectives (Wiley, 2013).

112. S.A. Antipov, Fast Transverse Beam Instability Caused by Electron Cloud Trapped in Combined Function Magnets (Springer, 2018).

113. R.W. Hamm, M.E. Hamm, Industrial Accelerators and Their Applications (World Scientific, 2012).

114. R. Van Grieken, A. Markowicz, Handbook of X-Ray Spectrometry (CRC Press, 2001).

115. J.E. Turner, Atoms, Radiation, and Radiation Protection, 3rd edn. (Wiley-VCH, 2007).

116. V.P. Kovalev, Secondary Radiation in Particle Accelerators (Atomizdat, Moscow, 1979). (in Russian)

117. C. Bungau et al., Induced activation in accelerator components. Phys. Rev. ST Accel. Beams 17, 084701 (2014)

118. K.M. Morehouseand V. Komolprasert Overview of Irradiation of Food and Packaging. In: ACS Symposium Series 875 Irradiation of Food and Packaging, 2004

119. D.A. Close, J.C. Pratt, H.F. Atwater, Development of an enrichment measurement technique and its application to enrichment verification of gaseous UF6. Nucl. Instrum. Methods Phys. Res. Sect. A 240(2), 398-405 (1985)

120. J. England, Longitudinal Shaping of Relativistic Electron Bunches Generated by an RF Photoinjector (University of California Los Angeles, Department of Physics and Astronomy, September 2007).

121. B.S. Ishkhanov, A.V. Poseryaev, V.I. Shvedunov, Dynamics of the Beam in High Current Electron Linear Accelerator (SINP MSU, Moscow, 2004). (in Russian)

122. S.V. Kutsaev, "Studies of accelerating structures for electron linear accelerators for cargo inspection. Ph.D. Thesis, NRNU MEPhI, 2011 (in Russian).

123. S.V. Kutsaev et al., Accelerating Structure for C-Band Electron Linear Accelerator Optimization. In: Proc. of LINAC08, Victoria, BC, Canada, p.THP058, 2008

124. G.V. Stupakov, Wake and impedance. AIP Conf. Proc. 592(1), 205-230 (2001)

125. K.L. Bane, P.B. Wilson, T. Weiland, Wake fields and wake field acceleration. AIP Conf. Proc. 127, 875-928 (1985)

126. K. Whitham et al., CRANE RF accelerator for high current radiation damage studies. In: Proc. 1992 linear accelerator conference, Canada, 1992

127. M.G. Kelliher, J.C. Nygard, A.J. Gale, The electron linear accelerator as a pulsed radiation source. IRE Trans. Nucl. Sci. 3(3), 1-7 (1956)

128. H. Padamsee, RF Superconductivity: Science, Technology, and Applications (Wiley-VCH, 2009).

129. D.S. Yurov et al., Continuous-wave electron linear accelerators for industrial applications. Phys. Rev. Accel. Beams 20, 044702 (2017)

130. https://www.radiasoft.net/

131. https://radiabeam.com/

132. G.A. Korn, T.M. Korn, Mathematical Handbook for Scientists and Engineers, 2nd edn. (Dover Publications, 2000). 